\title{
CONDITIONALLY BI-FREE INDEPENDENCE WITH AMALGAMATION
}

\author{
YINZHENG GU AND PAUL SKOUFRANIS
}

\begin{abstract}
In this paper, we introduce the notion of conditionally bi-free independence in an amalgamated setting. We define operator-valued conditionally bi-multiplicative pairs of functions and construct operatorvalued conditionally bi-free moment and cumulant functions. It is demonstrated that conditionally bifree independence with amalgamation is equivalent to the vanishing of mixed operator-valued bi-free and conditionally bi-free cumulants. Furthermore, an operator-valued conditionally bi-free partial $\mathcal{R}$-transform is constructed and various operator-valued conditionally bi-free limit theorems are studied.
\end{abstract}

\section{INTRODUCTION}

The notion of conditionally free (c-free for short) independence was introduced in 3 as a generalization of the notion of free independence to two-state systems. In our previous paper [ $[\underline{6}$ we introduced the notion of conditionally bi-free (c-bi-free for short) independence in order to study the non-commutative left and right actions of algebras on a reduced c-free product simultaneously. Thus conditional bi-freeness is an extension of the notion of bi-free independence 14 to two-state systems. Moreover 6] introduced c- $(\ell, r)$-cumulants and demonstrated that a family of pairs of algebras in a two-state non-commutative probability space is conditionally bi-free if and only if mixed $(\ell, r)$ - and $\mathrm{c}-(\ell, r)$-cumulants vanish.

In [13] Voiculescu generalized his own notion of free independence by replacing the scalars with an arbitrary algebra thereby obtaining the notion of free independence with amalgamation (see also [12] for the combinatorial aspects). For c-free independence, the generalization to an amalgamated setting over a pair of algebras was done by Popa in [9] (see also 8]). On the other hand, the framework for generalizing bi-free independence to an amalgamated setting was suggested by Voiculescu in [14, Section 8] and the theory was fully developed in 4 .

The main goal of this paper is to extend the notion of c-bi-free independence to an amalgamated setting over a pair of algebras. Furthermore, we demonstrate that the combinatorics of conditionally bi-free probability and bi-free probability with amalgamation, which are governed by the lattice of bi-non-crossing partitions, are specific instances of more general combinatorial structures.

Including this introduction this paper contains nine sections which are structured as follows. Section 2 briefly reviews some of the background material pertaining to conditionally bi-free probability and bi-free probability with amalgamation from [4 6]. In particular, the notions bi-non-crossing partitions and diagrams, their lateral refinements and cappings, interior and exterior blocks, $\mathcal{B}-\mathcal{B}$-non-commutative probability spaces, operator-valued bi-multiplicative functions, and the operator-valued bi-free moment and cumulant functions are recalled.

Section 3 introduces the structures studied within conditionally bi-free independence with amalgamation. We define the notion of a $\mathcal{B}-\mathcal{B}$-non-commutative probability space with a pair of $(\mathcal{B}, \mathcal{D})$-valued expectations $(\mathcal{A}, \mathbb{E}, \mathbb{F}, \varepsilon)$ (see Definition 3.4 ), demonstrate a representation of $\mathcal{A}$ as linear operators on a $\mathcal{B}$ - $\mathcal{B}$-bimodule with a pair of specified $(\mathcal{B}, \mathcal{D})$-valued states (see Theorem [3.5), and define the notion of conditionally bi-free independence with amalgamation over $(\mathcal{B}, \mathcal{D})$ thereby generalizing conditionally bi-free independence to the operator-valued setting and bi-free independence with amalgamation to the two-state setting.

Section 4 introduces the notion of an operator-valued conditionally bi-multiplicative pair of functions (see Definition 4.2). Each such pair consists of two functions where the first function is operator-valued bi-multiplicative (see [4, Definition 4.2.1]) and the second function is defined via a certain rule using the first

Date: August 6, 2018.

2010 Mathematics Subject Classification. Primary 46L54; Secondary 46L53.

Key words and phrases. conditionally bi-free independence, conditionally bi-multiplicativity, conditionally bi-free moment and cumulant pairs. 
function. Furthermore, operator-valued conditionally bi-free moment and cumulant pairs (see Definitions 4.4 and 4.7) are introduced and shown to be operator-valued conditionally bi-multiplicative.

Sections 5 and 6 provide alternate characterizations of conditionally bi-free independence with amalgamation. More precisely, Section 5 demonstrates through Theorem 5.5 that a family of pairs of $\mathcal{B}$-algebras in a $\mathcal{B}$ - $\mathcal{B}$-non-commutative probability space with a pair of $(\mathcal{B}, \mathcal{D})$-valued expectations $(\mathcal{A}, \mathbb{E}, \mathbb{F}, \varepsilon)$ is c-bi-free over $(\mathcal{B}, \mathcal{D})$ if and only if certain moment expressions with respect to $\mathbb{E}$ and $\mathbb{F}$ are satisfied. On the other hand, Section 6 demonstrates through Theorem 6.4 that a family of pairs of $\mathcal{B}$-algebras is c-bi-free over $(\mathcal{B}, \mathcal{D})$ if and only if their mixed operator-valued bi-free and conditionally bi-free cumulants vanish.

Section 7 provides additional properties such as the vanishing of operator-valued conditionally bi-free cumulants when a left or right $\mathcal{B}$-operator is input, how c-bi-free independence over $(\mathcal{B}, \mathcal{D})$ can be deduced from c-free independence over $(\mathcal{B}, \mathcal{D})$ under certain conditions, and how operator-valued conditionally bi-free cumulants involving products of operators may be computed.

In Section 8, an operator-valued conditionally bi-free partial $\mathcal{R}$-transform is constructed as the operatorvalued analogue of the conditionally bi-free partial $\mathcal{R}$-transform (see [6, Definition 5.3]). As with the operatorvalued bi-free partial $\mathcal{R}$-transform (see [11, Section 5]), the said transform is also a function of three $\mathcal{B}$ variables, and a formula relating it to the moment series is proved using combinatorics. Finally, in Section 9. operator-valued c-bi-free distributions are discussed and various operator-valued c-bi-free limit theorems are studied.

\section{Preliminaries}

In this section, we review the necessary background on conditionally bi-free probability and operatorvalued bi-free probability required for this paper.

2.1. Conditionally bi-free probability. We recall several definitions and results relating to conditionally bi-free probability. For more precision, see [6].

Definition 2.1. Let $(\mathcal{A}, \varphi, \psi)$ be a two-state non-commutative probability space; that is, $\mathcal{A}$ is a unital algebra and $\varphi, \psi: \mathcal{A} \rightarrow \mathbb{C}$ are unital linear functionals. A pair of algebras in $\mathcal{A}$ is an ordered pair $\left(A_{\ell}, A_{r}\right)$ of unital subalgebras of $\mathcal{A}$.

Definition 2.2. A family $\left\{\left(A_{k, \ell}, A_{k, r}\right)\right\}_{k \in K}$ of pairs of algebras in a two-state non-commutative probability space $(\mathcal{A}, \varphi, \psi)$ is said to be conditionally bi-freely independent (or $c$-bi-free for short) with respect to $(\varphi, \psi)$ if there is a family of two-state vector spaces with specified state-vectors $\left\{\left(\mathcal{X}_{k}, \mathcal{X}_{k}^{\circ}, \xi_{k}, \varphi_{k}\right)\right\}_{k \in K}$ and unital homomorphisms

$$
\ell_{k}: A_{k, \ell} \rightarrow \mathcal{L}\left(\mathcal{X}_{k}\right) \quad \text { and } \quad r_{k}: A_{k, r} \rightarrow \mathcal{L}\left(\mathcal{X}_{k}\right)
$$

such that the joint distribution of $\left\{\left(A_{k, \ell}, A_{k, r}\right)\right\}_{k \in K}$ with respect to $(\varphi, \psi)$ is equal to the joint distribution of the family

$$
\left\{\left(\lambda_{k} \circ \ell_{k}\left(A_{k, \ell}\right), \rho_{k} \circ r_{k}\left(A_{k, r}\right)\right)\right\}_{k \in K}
$$

in $\mathcal{L}(\mathcal{X})$ with respect to $\left(\varphi_{\xi}, \psi_{\xi}\right)$, where $\left(\mathcal{X}, \mathcal{X}^{\circ}, \xi, \varphi\right)=*_{k \in K}\left(\mathcal{X}_{k}, \mathcal{X}_{k}^{\circ}, \xi_{k}, \varphi_{k}\right)$.

In general, a map $\chi:\{1, \ldots, n\} \rightarrow\{\ell, r\}$ is used to designate whether the $k^{\text {th }}$ operator in a sequence of $n$ operators is a left operator (when $\chi(k)=\ell$ ) or a right operator (when $\chi(k)=r$ ), a map $\omega:\{1, \ldots, n\} \rightarrow I \sqcup J$ is used to designate the index of the $k^{\text {th }}$ operator, and a map $\omega:\{1, \ldots, n\} \rightarrow K$ is used to designate from which collection of operators the $k^{\text {th }}$ operator hails from.

Given $\omega:\{1, \ldots, n\} \rightarrow I \sqcup J$ for non-empty disjoint index sets $I$ and $J$, we define the corresponding map $\chi_{\omega}:\{1, \ldots, n\} \rightarrow\{\ell, r\}$ by

$$
\chi_{\omega}(k)=\left\{\begin{array}{ll}
\ell & \text { if } \omega(k) \in I \\
r & \text { if } \omega(k) \in J
\end{array} .\right.
$$

Given a map $\omega:\{1, \ldots, n\} \rightarrow K$, we may view $\omega$ as a partition of $\{1, \ldots, n\}$ with blocks $\left\{\omega^{-1}(\{k\})\right\}_{k \in K}$. Thus $\pi \leq \omega$ denotes $\pi$ is a refinement of the partition induced by $\omega$.

For the basic definitions and combinatorics of bi-free probability that will be used in this paper, we refer the reader to [4,5,7,14] or the summary given in [6, Section 2]. Particular attention should be paid to:

- the set $\mathcal{B N C}(\chi)$ of bi-non-crossing partitions with respect to $\chi:\{1, \ldots, n\} \rightarrow\{\ell, r\}$, and the minimal and maximal elements $0_{\chi}$ and $1_{\chi}$ of $\mathcal{B N C}(\chi)$ (see [4, Definition 2.1.1]); 
- for $m, n \geq 0$ with $m+n \geq 1,1_{m, n}$ denotes $1_{\chi_{m, n}}$ where $\chi_{m, n}:\{1, \ldots, m+n\} \rightarrow\{\ell, r\}$ is such that $\chi_{m, n}(k)=\ell$ if $k \leq m$ and $\chi_{m, n}(k)=r$ if $k>m$;

- the Möbius function $\mu_{\mathcal{B N C}}$ on the lattice of bi-non-crossing partitions (see [5, Remark 3.1.4]);

- the total ordering $\prec_{\chi}$ on $\{1, \ldots, n\}$ and the notion of $\chi$-interval induced by $\chi:\{1, \ldots, n\} \rightarrow\{\ell, r\}$ (see [4, Definition 4.1.1]);

- the set $\mathcal{L R}(\chi, \omega)$ of shaded $\mathcal{L R}$-diagrams corresponding to $\chi:\{1, \ldots, n\} \rightarrow\{\ell, r\}$ and $\omega:\{1, \ldots, n\} \rightarrow$ $K$, and the subsets $\mathcal{L R}_{k}(\chi, \omega)(1 \leq k \leq n)$ of $\mathcal{L R}(\chi, \omega)$ with exactly $k$ spines reaching the top (see [5, Section 2.5]);

- the notion $\leq_{\text {lat }}$ of lateral refinement (see [5. Definition 2.5.5]);

- the family $\left\{\kappa_{\chi}: \mathcal{A}^{n} \rightarrow \mathbb{C}\right\}_{n \geq 1, \chi:\{1, \ldots, n\} \rightarrow\{\ell, r\}}$ of $(\ell, r)$-cumulants (see [7, Definition 5.2]).

Inspired by the 'vanishing of mixed $(\ell, r)$-cumulants' characterization of bi-free independence and the 'vanishing of mixed free and c-free cumulants' characterization of c-free independence, we introduced in 6. Subsection 3.3] the family of c- $(\ell, r)$-cumulants using bi-non-crossing partitions that are divided into two types. More precisely, a block $V$ of a bi-non-crossing partition $\pi \in \mathcal{B N C}(\chi)$ is said to be interior if there exists another block $W$ of $\pi$ such that $\min _{\prec_{\chi}}(W) \prec_{\chi} \min _{\prec_{\chi}}(V)$ and $\max _{\prec_{\chi}}(V) \prec_{\chi} \max _{\prec_{\chi}}(W)$, where $\min _{\prec_{\chi}}$ and $\max _{\prec_{\chi}}$ denote the minimum and maximum elements with respect to $\prec_{\chi}$. A block of $\pi$ is said to be exterior if it is not interior. The family

$$
\left\{\mathcal{K}_{\chi}: \mathcal{A}^{n} \rightarrow \mathbb{C}\right\}_{n \geq 1, \chi:\{1, \ldots, n\} \rightarrow\{\ell, r\}}
$$

of $c$ - $(\ell, r)$-cumulants of a two-state non-commutative probability space $(\mathcal{A}, \varphi, \psi)$ is recursively defined by

$$
\varphi\left(a_{1} \cdots a_{n}\right)=\sum_{\pi \in \mathcal{B N C}(\chi)} \mathcal{K}_{\pi}\left(a_{1}, \ldots, a_{n}\right)
$$

where

$$
\mathcal{K}_{\pi}\left(a_{1}, \ldots, a_{n}\right)=\left(\prod_{\substack{V \in \pi \\ V \text { interior }}} \kappa_{\left.\chi\right|_{V}}\left(\left.\left(a_{1}, \ldots, a_{n}\right)\right|_{V}\right)\right)\left(\prod_{\substack{V \in \pi \\ V \text { exterior }}} \mathcal{K}_{\left.\chi\right|_{V}}\left(\left.\left(a_{1}, \ldots, a_{n}\right)\right|_{V}\right)\right),
$$

for all $n \geq 1, \chi:\{1, \ldots, n\} \rightarrow\{\ell, r\}$, and $a_{1}, \ldots, a_{n} \in \mathcal{A}$.

Furthermore, as noticed in [6, Section 4], in order to obtain a moment formula for conditionally bi-free independence, additional sets of shaded diagrams and terminology are required.

Definition 2.3. Let $n \geq 1, \chi:\{1, \ldots, n\} \rightarrow\{\ell, r\}$, and $\omega:\{1, \ldots, n\} \rightarrow K$ be given.

(1) For $0 \leq k \leq n$, let $\mathcal{L R}_{k}^{\text {lat }}(\chi, \omega)$ denote the set of all diagrams that can be obtained from $\mathcal{L R}_{k}(\chi, \omega)$ under later refinement (i.e., cutting spines that do not reach the top). For $D^{\prime} \in \mathcal{L R}_{k}^{\text {lat }}(\chi, \omega)$ and $D \in \mathcal{L R}_{k}(\chi, \omega)$, write $D \geq_{\text {lat }} D^{\prime}$ if $D^{\prime}$ can be obtained by laterally refining $D$. Moreover, let

$$
\mathcal{L R}^{\text {lat }}(\chi, \omega)=\bigcup_{k=0}^{n} \mathcal{L R}_{k}^{\text {lat }}(\chi, \omega)
$$

(2) Let $0 \leq k \leq n$ and $D \in \mathcal{L R}_{k}^{\text {lat }}(\chi, \omega)$. A diagram $D^{\prime}$ is said to be a capping of $D$, denoted $D \geq_{\text {cap }} D^{\prime}$, if $D^{\prime}=D$ or $D^{\prime}$ can be obtained by removing spines from $D$ that reach the top. Let $\mathcal{L R}_{m}^{\text {latcap }}(\chi, \omega)$ denote the set of all diagrams with $m$ spines reaching the top that can be obtained by capping some $D \in \mathcal{L R}_{k}^{\text {lat }}(\chi, \omega)$ with $k \geq m$. Moreover, let

$$
\mathcal{L R}^{\text {latcap }}(\chi, \omega)=\bigcup_{m=0}^{n} \mathcal{L R}_{m}^{\text {latcap }}(\chi, \omega)
$$

(3) For $D \in \mathcal{L R}_{m}^{\text {latcap }}(\chi, \omega)$, let $|D|=($ number of blocks of $D)+m$.

(4) Let $0 \leq m \leq n, k \geq m, D \in \mathcal{L R}_{k}(\chi, \omega)$, and $D^{\prime} \in \mathcal{L R}_{m}^{\text {latcap }}(\chi, \omega)$. We say that $D$ laterally caps to $D^{\prime}$, denoted $D \geq_{\text {latcap }} D^{\prime}$, if there exists $D^{\prime \prime} \in \mathcal{L R}_{k}^{\text {lat }}(\chi, \omega)$ such that $D \geq_{\text {lat }} D^{\prime \prime}$ and $D^{\prime \prime} \geq_{\text {cap }} D^{\prime}$.

Suppose $a_{1}, \ldots, a_{n}$ are elements in a two-state non-commutative probability space $(\mathcal{A}, \varphi, \psi)$, and $D \in$ $\mathcal{L R}^{\text {latcap }}(\chi, \omega)$ with blocks $V_{1}, \ldots, V_{p}$ whose spines do not reach the top and $W_{1}, \ldots, W_{q}$ whose spines reach 
the top. Writing $V_{i}=\left\{r_{i, 1}<\cdots<r_{i, s_{i}}\right\}$ and $W_{j}=\left\{r_{j, 1}<\cdots<r_{j, t_{j}}\right\}$, we define

$$
\varphi_{D}\left(a_{1}, \ldots, a_{n}\right)=\prod_{i=1}^{p} \psi\left(a_{r_{i, 1}} \cdots a_{r_{i, s_{i}}}\right) \prod_{j=1}^{q} \varphi\left(a_{r_{j, 1}} \cdots a_{r_{j, t_{j}}}\right) .
$$

Under the above notation, the following moment type characterization and vanishing of mixed cumulants characterization were established in [6, Theorems 4.1 and 4.8].

Theorem 2.4. A family $\left\{\left(A_{k, \ell}, A_{k, r}\right)\right\}_{k \in K}$ of pairs of algebras in a two-state non-commutative probability space $(\mathcal{A}, \varphi, \psi)$ is c-bi-free with respect to $(\varphi, \psi)$ if and only if

$$
\psi\left(a_{1} \cdots a_{n}\right)=\sum_{\pi \in \mathcal{B N \mathcal { N }}(\chi)}\left[\sum_{\substack{\sigma \in \mathcal{B N \mathcal { C }}(\chi) \\ \pi \leq \sigma \leq \omega}} \mu_{\mathcal{B N C}(\pi, \sigma)}\right] \psi_{\pi}\left(a_{1}, \ldots, a_{n}\right)
$$

and

$$
\varphi\left(a_{1} \cdots a_{n}\right)=\sum_{D \in \mathcal{L} \mathcal{R}^{\text {latcap }}(\chi, \omega)}\left[\sum_{\substack{D^{\prime} \in \mathcal{L} \mathcal{R}(\chi, \omega) \\ D^{\prime} \geq \text { latcap } D}}(-1)^{|D|-\left|D^{\prime}\right|}\right] \varphi_{D}\left(a_{1}, \ldots, a_{n}\right)
$$

for all $n \geq 1, \chi:\{1, \ldots, n\} \rightarrow\{\ell, r\}, \omega:\{1, \ldots, n\} \rightarrow K$, and $a_{1}, \ldots, a_{n} \in \mathcal{A}$ with $a_{k} \in A_{\omega(k), \chi(k)}$.

Equivalently, for all $n \geq 2, \chi:\{1, \ldots, n\} \rightarrow\{\ell, r\}, \omega:\{1, \ldots, n\} \rightarrow K$, and $a_{k}$ as above, we have

$$
\kappa_{\chi}\left(a_{1}, \ldots, a_{n}\right)=\mathcal{K}_{\chi}\left(a_{1}, \ldots, a_{n}\right)=0
$$

whenever $\omega$ is not constant.

2.2. Bi-free probability with amalgamation. Now we recall bi-free probability in an amalgamated setting. Since our constructions for operator-valued conditionally bi-free independence in Section 3 are very similar, we shall only present the essential concepts. Please refer to [4, Section 3] or the summary given in [11, Section 2] for complete details. In particular, the following definitions and results will be generalized:

- a $\mathcal{B}$ - $\mathcal{B}$-bimodule with a specified $\mathcal{B}$-valued state $\left(\mathcal{X}, \mathcal{X}^{\circ}, \mathfrak{p}\right)$ (see [4, Definition 3.1.1]);

- the free product with amalgamation over $\mathcal{B}$ of a family $\left\{\left(\mathcal{X}_{k}, \mathcal{X}_{k}^{\circ}, \mathfrak{p}_{k}\right)\right\}_{k \in K}$ of $\mathcal{B}$ - $\mathcal{B}$-bimodules with specified $\mathcal{B}$-valued states (see [4, Construction 3.1.7]);

- a $\mathcal{B}$ - $\mathcal{B}$-non-commutative probability space $(\mathcal{A}, \mathbb{E}, \varepsilon)$ with left and right algebras $\mathcal{A}_{\ell}$ and $\mathcal{A}_{r}$ (see [4, Definition 3.2.1]);

- any $\mathcal{B}$ - $\mathcal{B}$-non-commutative probability can be represented on a $\mathcal{B}$ - $\mathcal{B}$-bimodule with a specified $\mathcal{B}$ valued state (see [4, Theorem 3.2.4]).

Furthermore, in order to discuss operator-valued bi-free probability, one needs the correct notions for moment and cumulant functions, which we now review in greater depth.

Definition 2.5. Let $(\mathcal{A}, \mathbb{E}, \varepsilon)$ be a $\mathcal{B}$ - $\mathcal{B}$-non-commutative probability space and let

$$
\Psi: \bigcup_{n \geq 1} \bigcup_{\chi:\{1, \ldots, n\} \rightarrow\{\ell, r\}} \mathcal{B N C}(\chi) \times \mathcal{A}_{\chi(1)} \times \cdots \times \mathcal{A}_{\chi(n)} \rightarrow \mathcal{B}
$$

be a function that is linear in each $\mathcal{A}_{\chi(k)}$. We say that $\Psi$ is operator-valued bi-multiplicative if for every $\chi:\{1, \ldots, n\} \rightarrow\{\ell, r\}, Z_{k} \in \mathcal{A}_{\chi(k)}, b \in \mathcal{B}$, and $\pi \in \mathcal{B N C}(\chi)$, the following four conditions hold.

(1) Let

If $\chi(n)=\ell$, then

$$
q=\max \{k \in\{1, \ldots, n\} \mid \chi(k) \neq \chi(n)\} .
$$

$$
\Psi_{1_{\chi}}\left(Z_{1}, \ldots, Z_{n-1}, Z_{n} L_{b}\right)=\left\{\begin{array}{ll}
\Psi_{1_{\chi}}\left(Z_{1}, \ldots, Z_{q-1}, Z_{q} R_{b}, Z_{q+1}, \ldots, Z_{n}\right) & \text { if } q \neq-\infty \\
\Psi_{1_{\chi}}\left(Z_{1}, \ldots, Z_{n-1}, Z_{n}\right) b & \text { if } q=-\infty
\end{array} .\right.
$$

If $\chi(n)=r$, then

$$
\Psi_{1_{\chi}}\left(Z_{1}, \ldots, Z_{n-1}, Z_{n} R_{b}\right)=\left\{\begin{array}{ll}
\Psi_{1_{\chi}}\left(Z_{1}, \ldots, Z_{q-1}, Z_{q} L_{b}, Z_{q+1}, \ldots, Z_{n}\right) & \text { if } q \neq-\infty \\
b \Psi_{1_{\chi}}\left(Z_{1}, \ldots, Z_{n-1}, Z_{n}\right) & \text { if } q=-\infty
\end{array} .\right.
$$


(2) Let $p \in\{1, \ldots, n\}$, and let

$$
q=\max \{k \in\{1, \ldots, n\} \mid \chi(k)=\chi(p), k<p\} .
$$

If $\chi(p)=\ell$, then

$$
\Psi_{1_{\chi}}\left(Z_{1}, \ldots, Z_{p-1}, L_{b} Z_{p}, Z_{p+1}, \ldots, Z_{n}\right)=\left\{\begin{array}{ll}
\Psi_{1_{\chi}}\left(Z_{1}, \ldots, Z_{q-1}, Z_{q} L_{b}, Z_{q+1}, \ldots, Z_{n}\right) & \text { if } q \neq-\infty \\
b \Psi_{1_{\chi}}\left(Z_{1}, Z_{2}, \ldots, Z_{n}\right) & \text { if } q=-\infty
\end{array} .\right.
$$

If $\chi(p)=r$, then

$$
\Psi_{1_{\chi}}\left(Z_{1}, \ldots, Z_{p-1}, R_{b} Z_{p}, Z_{p+1}, \ldots, Z_{n}\right)=\left\{\begin{array}{ll}
\Psi_{1_{\chi}}\left(Z_{1}, \ldots, Z_{q-1}, Z_{q} R_{b}, Z_{q+1}, \ldots, Z_{n}\right) & \text { if } q \neq-\infty \\
\Psi_{1_{\chi}}\left(Z_{1}, Z_{2}, \ldots, Z_{n}\right) b & \text { if } q=-\infty
\end{array} .\right.
$$

(3) Suppose that $V_{1}, \ldots, V_{m}$ are $\chi$-intervals ordered by $\prec_{\chi}$ which partition $\{1, \ldots, n\}$, each a union of blocks of $\pi$. Then

$$
\Psi_{\pi}\left(Z_{1}, \ldots, Z_{n}\right)=\Psi_{\left.\pi\right|_{V_{1}}}\left(\left.\left(Z_{1}, \ldots, Z_{n}\right)\right|_{V_{1}}\right) \cdots \Psi_{\left.\pi\right|_{V_{m}}}\left(\left.\left(Z_{1}, \ldots, Z_{n}\right)\right|_{V_{m}}\right) .
$$

(4) Suppose that $V$ and $W$ partition $\{1, \ldots, n\}$, each a union of blocks of $\pi, V$ is a $\chi$-interval, and

$$
\min _{\prec x}(\{1, \ldots, n\}), \max _{\prec x}(\{1, \ldots, n\}) \in W .
$$

Let

$$
p=\max _{\prec \chi}\left(\left\{k \in W \mid k \prec_{\chi} \min _{\prec_{\chi}}(V)\right\}\right) \quad \text { and } \quad q=\min _{\prec_{\chi}}\left(\left\{k \in W \mid \max _{\prec_{\chi}}(V) \prec_{\chi} k\right\}\right) .
$$

Then

$$
\begin{aligned}
& \Psi_{\pi}\left(Z_{1}, \ldots, Z_{n}\right)=\left\{\begin{array}{l}
\Psi_{\left.\pi\right|_{W}} \\
\Psi_{\left.\pi\right|_{W}}
\end{array}\right\}\left(\begin{array}{ll}
\left.\left.\left(Z_{1}, \ldots, Z_{p-1}, Z_{p} L_{\Psi_{\left.\pi\right|_{V}}\left(\left.\left(Z_{1}, \ldots, Z_{n}\right)\right|_{V}\right)}, Z_{p+1}, \ldots, Z_{n}\right)\right|_{W}\right) & \text { if } \chi(p)=\ell \\
\left.\left.Z_{1}, \ldots, Z_{p-1}, R_{\Psi_{\left.\pi\right|_{V}}\left(\left.\left(Z_{1}, \ldots, Z_{n}\right)\right|_{V}\right)} Z_{p}, Z_{p+1}, \ldots, Z_{n}\right)\left.\right|_{W}\right) & \text { if } \chi(p)=r
\end{array}\right.
\end{aligned}
$$

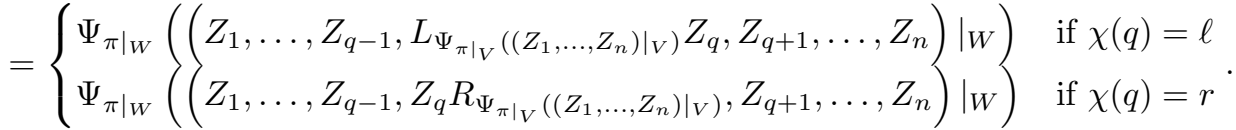

Given an operator-valued bi-multiplicative function, conditions (1) to (4) above are reduction properties which allows one to move $\mathcal{B}$-operators around and, more importantly, to compute the values on arbitrary bi-non-crossing partitions based on its values on full non-crossing partitions.

Finally, the two most important operator-valued bi-multiplicative functions in the theory, called operatorvalued bi-free moment and cumulant functions, are defined as follows.

Definition 2.6. Let $(\mathcal{A}, \mathbb{E}, \varepsilon)$ be a $\mathcal{B}$ - $\mathcal{B}$-non-commutative probability space. For $\chi:\{1, \ldots, n\} \rightarrow\{\ell, r\}$, $\pi \in \mathcal{B N C}(\chi)$, and $Z_{1}, \ldots, Z_{n} \in \mathcal{A}$, define $\mathbb{E}_{\pi}\left(Z_{1}, \ldots, Z_{n}\right) \in \mathcal{B}$ recursively as follows: Let $V$ be the block of $\pi$ that terminates closest to the bottom, so $\min (V)$ is largest among all blocks of $\pi$.

(1) If $\pi$ contains exactly one block (that is, $\left.\pi=1_{\chi}\right)$, define $\mathbb{E}_{1_{\chi}}\left(Z_{1}, \ldots, Z_{n}\right)=\mathbb{E}\left(Z_{1} \cdots Z_{n}\right)$.

(2) If $V=\{k+1, \ldots, n\}$ for some $k \in\{1, \ldots, n-1\}$ (so $\min (V)$ is not adjacent to any spine of $\pi$ ), define

$$
\mathbb{E}_{\pi}\left(Z_{1}, \ldots, Z_{n}\right)=\left\{\begin{array}{ll}
\mathbb{E}_{\left.\pi\right|_{V} \mathrm{C}}\left(Z_{1}, \ldots, Z_{k} L_{\mathbb{E}_{\left.\pi\right|_{V}}\left(Z_{k+1}, \ldots, Z_{n}\right)}\right) & \text { if } \chi(\min (V))=\ell \\
\mathbb{E}_{\left.\pi\right|_{V} \mathrm{C}}\left(Z_{1}, \ldots, Z_{k} R_{\mathbb{E}_{\left.\pi\right|_{V}}\left(Z_{k+1}, \ldots, Z_{n}\right)}\right) & \text { if } \chi(\min (V))=r
\end{array} .\right.
$$

(3) Otherwise, $\min (V)$ is adjacent to a spine. Let $W$ denote the block of $\pi$ corresponding to the spine adjacent to $\min (V)$ and let $k$ be the smallest element of $W$ that is larger than $\min (V)$. Define

$$
\mathbb{E}_{\pi}\left(Z_{1}, \ldots, Z_{n}\right)=\left\{\begin{array}{ll}
\mathbb{E}_{\left.\pi\right|_{V} \mathrm{C}}\left(\left.\left(Z_{1}, \ldots, Z_{k-1}, L_{\mathbb{E}_{\left.\pi\right|_{V}}\left(\left.\left(Z_{1}, \ldots, Z_{n}\right)\right|_{V}\right)} Z_{k}, Z_{k+1}, \ldots, Z_{n}\right)\right|_{V^{\mathrm{C}}}\right) & \text { if } \chi(\min (V))=\ell \\
\mathbb{E}_{\left.\pi\right|_{V} \mathrm{C}}\left(\left.\left(Z_{1}, \ldots, Z_{k-1}, R_{\mathbb{E}_{\left.\pi\right|_{V}}\left(\left.\left(Z_{1}, \ldots, Z_{n}\right)\right|_{V}\right)} Z_{k}, Z_{k+1}, \ldots, Z_{n}\right)\right|_{V^{\mathrm{C}}}\right) & \text { if } \chi(\min (V))=r
\end{array} .\right.
$$

Definition 2.7. Let $(\mathcal{A}, \mathbb{E}, \varepsilon)$ be a $\mathcal{B}$ - $\mathcal{B}$-non-commutative probability space. The operator-valued bi-free moment and cumulant functions on $\mathcal{A}$ are

$$
\mathcal{E}, \kappa: \bigcup_{n \geq 1} \bigcup_{\chi:\{1, \ldots, n\} \rightarrow\{\ell, r\}} \mathcal{B N C}(\chi) \times \mathcal{A}_{\chi(1)} \times \cdots \times \mathcal{A}_{\chi(n)} \rightarrow \mathcal{B}
$$


defined by

$$
\mathcal{E}_{\pi}\left(Z_{1}, \ldots, Z_{n}\right)=\mathbb{E}_{\pi}\left(Z_{1}, \ldots, Z_{n}\right) \quad \text { and } \quad \kappa_{\pi}\left(Z_{1}, \ldots, Z_{n}\right)=\sum_{\substack{\sigma \in \mathcal{B N C}(\chi) \\ \sigma \leq \pi}} \mathcal{E}_{\sigma}\left(Z_{1}, \ldots, Z_{n}\right) \mu_{\mathcal{B N C}}(\sigma, \pi)
$$

for all $\chi:\{1, \ldots, n\} \rightarrow\{\ell, r\}, \pi \in \mathcal{B N C}(\chi)$, and $Z_{k} \in \mathcal{A}_{\chi(k)}$.

A substantial amount of effort was taken in [4, Sections 5 and 6 ] to show that both $\mathcal{E}$ and $\kappa$ are operatorvalued bi-multiplicative.

\section{Conditionally Bi-Free families With AMAlgamation}

In this section, we develop the structures to discuss conditionally bi-free independence with amalgamation. To begin, we need an analogue of a two-state vector space with a specified state-vector.

Definition 3.1. A $\mathcal{B}$ - $\mathcal{B}$-bimodule with a pair of specified $(\mathcal{B}, \mathcal{D})$-valued states is a quadruple $\left(\mathcal{X}, \mathcal{X}^{\circ}, \mathfrak{p}, \mathfrak{q}\right)$, where $\mathcal{B}$ and $\mathcal{D}$ are unital algebras such that $1:=1_{\mathcal{D}} \in \mathcal{B} \subset \mathcal{D}, \mathcal{X}$ is a direct sum of $\mathcal{B}$ - $\mathcal{B}$-bimodules $\mathcal{X}=\mathcal{B} \oplus \mathcal{X}^{\circ}, \mathfrak{p}: \mathcal{X} \rightarrow \mathcal{B}$ is the linear map $\mathfrak{p}(b \oplus \eta)=b$, and $\mathfrak{q}: \mathcal{X} \rightarrow \mathcal{D}$ is a linear $\mathcal{B}$ - $\mathcal{B}$-bimodule map such that $\mathfrak{q}(1 \oplus 0)=1$.

Given a $\mathcal{B}$ - $\mathcal{B}$-bimodule with a pair of specified $(\mathcal{B}, \mathcal{D})$-valued states $\left(\mathcal{X}, \mathcal{X}^{\circ}, \mathfrak{p}, \mathfrak{q}\right)$, we have

$$
\mathfrak{p}\left(b_{1} \cdot x \cdot b_{2}\right)=b_{1} \mathfrak{p}(x) b_{2} \quad \text { and } \quad \mathfrak{q}\left(b_{1} \cdot x \cdot b_{2}\right)=b_{1} \mathfrak{q}(x) b_{2}
$$

for all $b_{1}, b_{2} \in \mathcal{B}$ and $x \in \mathcal{X}$. Moreover, let $\mathcal{L}(\mathcal{X})$ denote the set of linear operators on $\mathcal{X}$, and recall from [4. Definition 3.1.3] that the operators $L_{b}, R_{b} \in \mathcal{L}(\mathcal{X})$ are defined by

$$
L_{b}(x)=b \cdot x \quad \text { and } \quad R_{b}(x)=x \cdot b
$$

for all $b \in \mathcal{B}$ and $x \in \mathcal{X}$. In addition, the left and right algebras of $\mathcal{L}(\mathcal{X})$ are the unital subalgebras $\mathcal{L}_{\ell}(\mathcal{X})$ and $\mathcal{L}_{r}(X)$ defined by

$$
\mathcal{L}_{\ell}(X)=\left\{Z \in \mathcal{L}(\mathcal{X}) \mid Z R_{b}=R_{b} Z \text { for all } b \in \mathcal{B}\right\}
$$

and

respectively.

As we are interested in conditionally bi-free independence with amalgamation, we need two expectations on $\mathcal{L}(\mathcal{X})$, one onto $\mathcal{B}$ and one to $\mathcal{D}$.

Definition 3.2. Given a $\mathcal{B}-\mathcal{B}$-bimodule with a pair of specified $(\mathcal{B}, \mathcal{D})$-valued states $\left(\mathcal{X}, \mathcal{X}^{\circ}, \mathfrak{p}, \mathfrak{q}\right)$, define the unital linear maps $\mathbb{E}_{\mathcal{L}(\mathcal{X})}: \mathcal{L}(\mathcal{X}) \rightarrow \mathcal{B}$ and $\mathbb{F}_{\mathcal{L}(\mathcal{X})}: \mathcal{L}(\mathcal{X}) \rightarrow \mathcal{D}$ by

$$
\mathbb{E}_{\mathcal{L}(\mathcal{X})}(Z)=\mathfrak{p}(Z(1 \oplus 0)) \quad \text { and } \quad \mathbb{F}_{\mathcal{L}(\mathcal{X})}(Z)=\mathfrak{q}(Z(1 \oplus 0))
$$

for all $Z \in \mathcal{L}(\mathcal{X})$. We call $\mathbb{E}_{\mathcal{L}(\mathcal{X})}$ and $\mathbb{F}_{\mathcal{L}(\mathcal{X})}$ the expectations of $\mathcal{L}(\mathcal{X})$ to $\mathcal{B}$ and $\mathcal{D}$, respectively.

There are specific properties of these expectations we wish to model.

Proposition 3.3. Let $\left(\mathcal{X}, \mathcal{X}^{\circ}, \mathfrak{p}, \mathfrak{q}\right)$ be a $\mathcal{B}$-B-bimodule with a pair of specified $(\mathcal{B}, \mathcal{D})$-valued states. We have

$$
\mathbb{E}_{\mathcal{L}(\mathcal{X})}\left(L_{b_{1}} R_{b_{2}} Z\right)=b_{1} \mathbb{E}_{\mathcal{L}(\mathcal{X})}(Z) b_{2}, \quad \mathbb{E}_{\mathcal{L}(\mathcal{X})}\left(Z L_{b}\right)=\mathbb{E}_{\mathcal{L}(\mathcal{X})}\left(Z R_{b}\right)
$$

and

$$
\mathbb{F}_{\mathcal{L}(\mathcal{X})}\left(L_{b_{1}} R_{b_{2}} Z\right)=b_{1} \mathbb{F}_{\mathcal{L}(\mathcal{X})}(Z) b_{2}, \quad \mathbb{F}_{\mathcal{L}(\mathcal{X})}\left(Z L_{b}\right)=\mathbb{F}_{\mathcal{L}(\mathcal{X})}\left(Z R_{b}\right)
$$

for all $b_{1}, b_{2}, b \in \mathcal{B}$ and $Z \in \mathcal{L}(\mathcal{X})$.

Proof. The results regarding $\mathbb{E}_{\mathcal{L}(\mathcal{X})}$ were shown in [4, Proposition 3.1.6]. Moreover, it is immediate that $\mathbb{F}_{\mathcal{L}(\mathcal{X})}\left(Z L_{b}\right)=\mathbb{F}_{\mathcal{L}(\mathcal{X})}\left(Z R_{b}\right)$ for all $b \in \mathcal{B}$ and $Z \in \mathcal{L}(\mathcal{X})$ as $L_{b}(1 \oplus 0)=R_{b}(1 \oplus 0)$. Finally, since $\mathfrak{q}$ is a linear $\mathcal{B}$ - $\mathcal{B}$-bimodule map, we have

$$
\mathbb{F}_{\mathcal{L}(\mathcal{X})}\left(L_{b_{1}} R_{b_{2}} Z\right)=\mathfrak{q}\left(L_{b_{1}} R_{b_{2}} Z(1 \oplus 0)\right)=b_{1} \mathfrak{q}(Z(1 \oplus 0)) b_{2}=b_{1} \mathbb{F}_{\mathcal{L}(\mathcal{X})}(Z) b_{2}
$$

for all $b_{1}, b_{2} \in \mathcal{B}$ and $Z \in \mathcal{L}(\mathcal{X})$. 
Given the above definition and proposition, we extend the notion of a two-state non-commutative probability space $(\mathcal{A}, \varphi, \psi)$ to the operator-valued setting as follows. Note this is also a natural extension of the notion of a $\mathcal{B}$ - $\mathcal{B}$-non-commutative probability space $(\mathcal{A}, \mathbb{E}, \varepsilon)$ from $[4$, Definition 3.2 .1$]$ to the two-state setting.

Definition 3.4. A $\mathcal{B}-\mathcal{B}$-non-commutative probability space with a pair of $(\mathcal{B}, \mathcal{D})$-valued expectations is a quadruple $(\mathcal{A}, \mathbb{E}, \mathbb{F}, \varepsilon)$, where $\mathcal{A}, \mathcal{B}$, and $\mathcal{D}$ are unital algebras such that $1:=1_{\mathcal{D}} \in \mathcal{B} \subset \mathcal{D}, \varepsilon: \mathcal{B} \otimes \mathcal{B}^{\text {op }} \rightarrow \mathcal{A}$ is a unital homomorphism such that $\left.\varepsilon\right|_{\mathcal{B} \otimes 1}$ and $\left.\varepsilon\right|_{1 \otimes \mathcal{B}^{\circ p}}$ are injective, and $\mathbb{E}: \mathcal{A} \rightarrow \mathcal{B}$ and $\mathbb{F}: \mathcal{A} \rightarrow \mathcal{D}$ are unital linear maps such that

$$
\mathbb{E}\left(\varepsilon\left(b_{1} \otimes b_{2}\right) Z\right)=b_{1} \mathbb{E}(Z) b_{2}, \quad \mathbb{E}(Z \varepsilon(b \otimes 1))=\mathbb{E}(Z \varepsilon(1 \otimes b))
$$

and

$$
\mathbb{F}\left(\varepsilon\left(b_{1} \otimes b_{2}\right) Z\right)=b_{1} \mathbb{F}(Z) b_{2}, \quad \mathbb{F}(Z \varepsilon(b \otimes 1))=\mathbb{F}(Z \varepsilon(1 \otimes b))
$$

for all $b_{1}, b_{2}, b \in \mathcal{B}$ and $Z \in \mathcal{A}$. Moreover, the unital subalgebras $\mathcal{A}_{\ell}$ and $\mathcal{A}_{r}$ of $\mathcal{A}$ defined by

$$
\mathcal{A}_{\ell}=\{Z \in \mathcal{A} \mid Z \varepsilon(1 \otimes b)=\varepsilon(1 \otimes b) Z \text { for all } b \in \mathcal{B}\}
$$

and

$$
\mathcal{A}_{r}=\{Z \in \mathcal{A} \mid Z \varepsilon(b \otimes 1)=\varepsilon(b \otimes 1) Z \text { for all } b \in \mathcal{B}\}
$$

will be called the left and right algebras of $\mathcal{A}$ respectively.

As with the bi-free case (see [4, Remark 3.2.2]), if $\left(\mathcal{X}, \mathcal{X}^{\circ}, \mathfrak{p}, \mathfrak{q}\right)$ is a $\mathcal{B}$ - $\mathcal{B}$-bimodule with a pair of specified $(\mathcal{B}, \mathcal{D})$-valued states, then we see via Proposition 3.3 that $\left(\mathcal{L}(\mathcal{X}), \mathbb{E}_{\mathcal{L}(\mathcal{X})}, \mathbb{F}_{\mathcal{L}(\mathcal{X})}, \varepsilon\right)$ is a $\mathcal{B}$ - $\mathcal{B}$-non-commutative probability space with a pair of $(\mathcal{B}, \mathcal{D})$-valued expectations where $\varepsilon: \mathcal{B} \otimes \mathcal{B}^{\text {op }} \rightarrow \mathcal{L}(\mathcal{X})$ is defined by $\varepsilon\left(b_{1} \otimes b_{2}\right)=$ $L_{b_{1}} R_{b_{2}}$. Moreover, the following result demonstrates that any $\mathcal{B}-\mathcal{B}$-non-commutative probability space with a pair of $(\mathcal{B}, \mathcal{D})$-valued expectations can be represented as linear operators on some $\left(\mathcal{X}, \mathcal{X}^{\circ}, \mathfrak{p}, \mathfrak{q}\right)$. Hence Definition 3.4 is the natural extension of [4, Definition 3.2.1]. As such, we will write $L_{b}$ and $R_{b}$ instead of $\varepsilon(b \otimes 1)$ and $\varepsilon(1 \otimes b)$ and refer to these as left and right $\mathcal{B}$-operators, respectively.

Theorem 3.5. If $\left(\mathcal{A}, \mathbb{E}_{\mathcal{A}}, \mathbb{F}_{\mathcal{A}}, \varepsilon\right)$ is a $\mathcal{B}$-B-non-commutative probability space with a pair of $(\mathcal{B}, \mathcal{D})$-valued expectations, then there exist a $\mathcal{B}$-B-bimodule with a pair of specified $(\mathcal{B}, \mathcal{D})$-valued states $\left(\mathcal{X}, \mathcal{X}^{\circ}, \mathfrak{p}, \mathfrak{q}\right)$ and a unital homomorphism $\theta: \mathcal{A} \rightarrow \mathcal{L}(\mathcal{X})$ such that

$$
\begin{gathered}
\theta\left(L_{b_{1}} R_{b_{2}}\right)=L_{b_{1}} R_{b_{2}}, \quad \theta\left(\mathcal{A}_{\ell}\right) \subset \mathcal{L}_{\ell}(\mathcal{X}), \quad \theta\left(\mathcal{A}_{r}\right) \subset \mathcal{L}_{r}(\mathcal{X}), \\
\mathbb{E}_{\mathcal{L}(\mathcal{X})}(\theta(Z))=\mathbb{E}_{\mathcal{A}}(Z), \quad \text { and } \quad \mathbb{F}_{\mathcal{L}(\mathcal{X})}(\theta(Z))=\mathbb{F}_{\mathcal{A}}(Z)
\end{gathered}
$$

for all $b_{1}, b_{2} \in \mathcal{B}$ and $Z \in \mathcal{A}$.

Proof. As shown in the proof of [4, Theorem 3.2.4], consider $\mathcal{X}=\mathcal{B} \oplus \mathcal{Y}$ as a vector space over $\mathbb{C}$ where

$$
\mathcal{Y}=\operatorname{ker}\left(\mathbb{E}_{\mathcal{A}}\right) / \operatorname{span}\left\{Z L_{b}-Z R_{b} \mid Z \in \mathcal{A}, b \in \mathcal{B}\right\} .
$$

Define $\theta: \mathcal{A} \rightarrow \mathcal{L}(\mathcal{X})$ by

$$
\theta(Z)(b)=\mathbb{E}_{\mathcal{A}}\left(Z L_{b}\right) \oplus \pi\left(Z L_{b}-L_{\mathbb{E}_{\mathcal{A}}\left(Z L_{b}\right)}\right), \quad b \in \mathcal{B},
$$

and

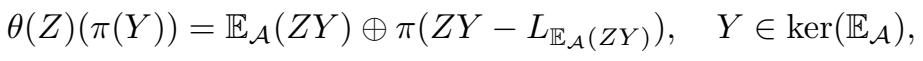

where $\pi: \operatorname{ker}\left(\mathbb{E}_{\mathcal{A}}\right) \rightarrow \mathcal{Y}$ denotes the canonical quotient map. It was shown in [4, Theorem 3.2.4] that $\theta$ is a unital homomorphism and $\mathcal{X}$ is a $\mathcal{B}$ - $\mathcal{B}$-bimodule via

$$
b \cdot \xi=\theta\left(L_{b}\right)(\xi) \quad \text { and } \quad \xi \cdot b=\theta\left(R_{b}\right)(\xi)
$$

for all $b \in \mathcal{B}$ and $\xi \in \mathcal{X}$. Thus we can define a specified $\mathcal{B}$-valued state $\mathfrak{p}$ on $\mathcal{X}$ by $\mathfrak{p}(b \oplus \pi(Y))=b$ for all $b \in \mathcal{B}$ and $\pi(Y) \in \mathcal{Y}$. Using this specified $\mathcal{B}$-valued state, we obtain that $\theta\left(\mathcal{A}_{\ell}\right) \subset \mathcal{L}_{\ell}(\mathcal{X}), \theta\left(\mathcal{A}_{r}\right) \subset \mathcal{L}_{r}(\mathcal{X})$, and $\mathbb{E}_{\mathcal{L}(\mathcal{X})}(\theta(Z))=\mathbb{E}_{\mathcal{A}}(Z)$.

On the other hand, since $\mathbb{F}_{\mathcal{A}}\left(Z L_{b}-Z R_{b}\right)=0$ for all $Z \in \mathcal{A}$ and $b \in \mathcal{B}$, there exists a unique linear map $\widetilde{\mathfrak{q}}: \mathcal{Y} \rightarrow \mathcal{D}$ such that $\left.\mathbb{F}_{\mathcal{A}}\right|_{\operatorname{ker}\left(\mathbb{E}_{\mathcal{A}}\right)}=\widetilde{\mathfrak{q}} \circ \pi$. Let $\mathfrak{q}: \mathcal{X} \rightarrow \mathcal{D}$ be the linear map defined by

$$
\mathfrak{q}(b \oplus \pi(Y))=b+\widetilde{\mathfrak{q}} \circ \pi(Y), \quad b \in \mathcal{B}, \quad \pi(Y) \in \mathcal{Y} .
$$


Then $\mathfrak{q}(1 \oplus 0)=1$ and

$$
\begin{aligned}
\mathfrak{q}\left(b_{1} \cdot(b \oplus \pi(Y)) \cdot b_{2}\right) & =\mathfrak{q}\left(\theta\left(L_{b_{1}}\right) \theta\left(R_{b_{2}}\right)(b \oplus \pi(Y))\right) \\
& =\mathfrak{q}\left(\theta\left(L_{b_{1}}\right)\left(b b_{2} \oplus \pi\left(R_{b_{2}} Y\right)\right)\right) \\
& =\mathfrak{q}\left(b_{1} b b_{2} \oplus \pi\left(L_{b_{2}} R_{b_{2}} Y\right)\right) \\
& =b_{1} b b_{2}+\mathbb{F}_{\mathcal{A}}\left(L_{b_{1}} R_{b_{2}} Y\right) \\
& =b_{1}\left(b+\mathbb{F}_{\mathcal{A}}(Y)\right) b_{2} \\
& =b_{1} \mathfrak{q}(b \oplus \pi(Y)) b_{2}
\end{aligned}
$$

for all $b_{1}, b_{2}, b \in \mathcal{B}$ and $\pi(Y) \in \mathcal{Y}$. Therefore, the quadruple $(\mathcal{X}, \mathcal{Y}, \mathfrak{p}, \mathfrak{q})$ is a $\mathcal{B}$ - $\mathcal{B}$-bimodule with a pair of specified $(\mathcal{B}, \mathcal{D})$-valued states. Finally, we have

$$
\mathbb{F}_{\mathcal{L}(\mathcal{X})}(\theta(Z))=\mathfrak{q}(\theta(Z)(1 \oplus 0))=\mathfrak{q}\left(\mathbb{E}_{\mathcal{A}}(Z) \oplus \pi\left(Z-L_{\mathbb{E}_{\mathcal{A}}(Z)}\right)\right)=\mathbb{E}_{\mathcal{A}}(Z)+\mathbb{F}_{\mathcal{A}}\left(Z-L_{\mathbb{E}_{\mathcal{A}}(Z)}\right)=\mathbb{F}_{\mathcal{A}}(Z)
$$

for all $Z \in \mathcal{A}$.

The next step is to extend the construction of the free product with amalgamation over $\mathcal{B}$ of a family $\left\{\left(\mathcal{X}_{k}, \mathcal{X}_{k}^{\circ}, \mathfrak{p}_{k}\right)\right\}_{k \in K}$ of $\mathcal{B}$ - $\mathcal{B}$-bimodules with specified $\mathcal{B}$-valued states (see [4, Construction 3.1.7]) to the current framework.

Construction 3.6. Let $\left\{\left(\mathcal{X}_{k}, \mathcal{X}_{k}^{\circ}, \mathfrak{p}_{k}, \mathfrak{q}_{k}\right)\right\}_{k \in K}$ be a family of $\mathcal{B}$ - $\mathcal{B}$-bimodules with pairs of specified $(\mathcal{B}, \mathcal{D})$ valued states. The $c$-free product of $\left\{\left(\mathcal{X}_{k}, \mathcal{X}_{k}^{\circ}, \mathfrak{p}_{k}, \mathfrak{q}_{k}\right)\right\}_{k \in K}$ with amalgamation over $(\mathcal{B}, \mathcal{D})$ is defined to be the $\mathcal{B}$ - $\mathcal{B}$-bimodule with a pair of specified $(\mathcal{B}, \mathcal{D})$-valued states $\left(\mathcal{X}, \mathcal{X}^{\circ}, \mathfrak{p}, \mathfrak{q}\right)$, where $\mathcal{X}=\mathcal{B} \oplus \mathcal{X}^{\circ}, \mathcal{X}^{\circ}$ is the $\mathcal{B}$ - $\mathcal{B}$-bimodule

$$
\mathcal{X}^{\circ}=\bigoplus_{n \geq 1}\left(\bigoplus_{k_{1} \neq \cdots \neq k_{n}} \mathcal{X}_{k_{1}}^{\circ} \otimes_{\mathcal{B}} \cdots \otimes_{\mathcal{B}} \mathcal{X}_{k_{n}}^{\circ}\right)
$$

with left and right actions of $\mathcal{B}$ on $\mathcal{X}^{\circ}$ defined by

$$
b \cdot\left(x_{1} \otimes \cdots \otimes x_{n}\right)=\left(b \cdot x_{1}\right) \otimes x_{2} \otimes \cdots \otimes x_{n} \quad \text { and } \quad\left(x_{1} \otimes \cdots \otimes x_{n}\right) \cdot b=x_{1} \otimes \cdots \otimes x_{n-1} \otimes\left(x_{n} \cdot b\right),
$$

respectively, $\mathfrak{p}: \mathcal{X} \rightarrow \mathcal{B}$ is the linear map $\mathfrak{p}(b \oplus \eta)=b$, and $\mathfrak{q}: \mathcal{X} \rightarrow \mathcal{D}$ is the linear $\mathcal{B}$ - $\mathcal{B}$-bimodule map such that $\mathfrak{q}(1 \oplus 0)=1$ and

$$
\mathfrak{q}\left(x_{1} \otimes \cdots \otimes x_{n}\right)=\mathfrak{q}_{k_{1}}\left(x_{1}\right) \cdots \mathfrak{q}_{k_{n}}\left(x_{n}\right)
$$

for $x_{1} \otimes \cdots \otimes x_{n} \in \mathcal{X}_{k_{1}}^{\circ} \otimes_{\mathcal{B}} \cdots \otimes_{\mathcal{B}} \mathcal{X}_{k_{n}}^{\circ}$ (note $\mathfrak{q}$ is well-defined as each $\mathfrak{q}_{k}$ is a linear $\mathcal{B}$ - $\mathcal{B}$-bimodule map).

For every $k \in K$, let

$$
V_{k}: \mathcal{X} \rightarrow \mathcal{X}_{k} \otimes_{\mathcal{B}}\left(\mathcal{B} \oplus \bigoplus_{n \geq 1}\left(\bigoplus_{\substack{k_{1} \neq \cdots \neq k_{n} \\ k_{1} \neq k}} \mathcal{X}_{k_{1}}^{\circ} \otimes_{\mathcal{B}} \cdots \otimes_{\mathcal{B}} \mathcal{X}_{k_{n}}^{\circ}\right)\right)
$$

be the standard $\mathcal{B}$ - $\mathcal{B}$-bimodule isomorphism, and define the left representation $\lambda_{k}: \mathcal{L}\left(\mathcal{X}_{k}\right) \rightarrow \mathcal{L}(\mathcal{X})$ by

$$
\lambda_{k}(Z)=V_{k}^{-1}(Z \otimes I) V_{k}
$$

for $Z \in \mathcal{L}\left(\mathcal{X}_{k}\right)$. Similarly, let

$$
W_{k}: \mathcal{X} \rightarrow\left(\mathcal{B} \oplus \bigoplus_{n \geq 1}\left(\bigoplus_{\begin{array}{c}
n \neq \cdots k_{n} \\
k_{1} \neq \cdots \neq k
\end{array}} \mathcal{X}_{k_{1}}^{\circ} \otimes_{\mathcal{B}} \cdots \otimes_{\mathcal{B}} \mathcal{X}_{k_{n}}^{\circ}\right)\right) \otimes_{\mathcal{B}} \mathcal{X}_{k}
$$

be the standard $\mathcal{B}$ - $\mathcal{B}$-bimodule isomorphism, and define the right representation $\rho_{k}: \mathcal{L}\left(\mathcal{X}_{k}\right) \rightarrow \mathcal{L}(\mathcal{X})$ by

$$
\rho_{k}(Z)=W_{k}^{-1}(I \otimes Z) W_{k}
$$

for $Z \in \mathcal{L}\left(\mathcal{X}_{k}\right)$. For the exact formulae of how $\lambda_{k}(Z)$ and $\rho_{k}(Z)$ act on $\mathcal{X}$, we refer to [4, Construction 3.1.7]. Note also that

$$
\mathbb{E}_{\mathcal{L}(\mathcal{X})}\left(\lambda_{k}(Z)\right)=\mathbb{E}_{\mathcal{L}(\mathcal{X})}\left(\rho_{k}(Z)\right)=\mathbb{E}_{\mathcal{L}\left(\mathcal{X}_{k}\right)}(Z) \quad \text { and } \quad \mathbb{F}_{\mathcal{L}(\mathcal{X})}\left(\lambda_{k}(Z)\right)=\mathbb{F}_{\mathcal{L}(\mathcal{X})}\left(\rho_{k}(Z)\right)=\mathbb{F}_{\mathcal{L}\left(\mathcal{X}_{k}\right)}(Z)
$$

for all $Z \in \mathcal{L}\left(\mathcal{X}_{k}\right)$. 
Remark 3.7. It is clear that that all of the above discussions hold if $\mathcal{B}=\mathcal{D}$. However, the more general setting that $\mathcal{B} \subset \mathcal{D}$ is desired due to a result of Boca [2. Indeed, suppose $\left\{\mathcal{A}_{k}\right\}_{k \in K}$ is a family of unital $C^{*}$ algebras containing $\mathcal{B}$ as a common $C^{*}$-subalgebra with $1_{\mathcal{A}_{k}} \in \mathcal{B}, \mathcal{D}$ is a unital $C^{*}$-algebra with $1_{\mathcal{D}} \in \mathcal{B} \subset \mathcal{D}$, and each $\mathcal{A}_{k}$ is endowed with two positive conditional expectations $\Psi_{k}: \mathcal{A}_{k} \rightarrow \mathcal{B}$ and $\Phi_{k}: \mathcal{A}_{k} \rightarrow \mathcal{D}$ such that $\mathcal{A}_{k}=\mathcal{B} \oplus \mathcal{A}_{k}^{\circ}$, where $\mathcal{A}_{k}^{\circ}=\operatorname{ker}\left(\Psi_{k}\right)$, as a direct sum of $\mathcal{B}$ - $\mathcal{B}$-bimodules.

Let $\mathcal{A}=\left(*_{\mathcal{B}}\right)_{k \in K} \mathcal{A}_{k}$ be the free product of $\left\{\mathcal{A}_{k}\right\}_{k \in K}$ with amalgamation over $\mathcal{B}$ (which can be identified as

$$
\mathcal{A}=\mathcal{B} \oplus \bigoplus_{n \geq 1}\left(\bigoplus_{k_{1} \neq \cdots \neq k_{n}} \mathcal{A}_{k_{1}}^{\circ} \otimes_{\mathcal{B}} \cdots \otimes_{\mathcal{B}} \mathcal{A}_{k_{n}}^{\circ}\right)
$$

as $\mathcal{B}$ - $\mathcal{B}$-bimodules), let $\Psi=\left(*_{\mathcal{B}}\right)_{k \in K} \Psi_{k}$ be the amalgamated free product of $\left\{\Psi_{k}\right\}_{k \in K}$, and let $\Phi: \mathcal{A} \rightarrow \mathcal{D}$ be the unital linear $\mathcal{B}$ - $\mathcal{B}$-bimodule map defined by

$$
\Phi\left(a_{1} \otimes \cdots \otimes a_{n}\right)=\Phi_{k_{1}}\left(a_{1}\right) \cdots \Phi_{k_{n}}\left(a_{n}\right)
$$

for $a_{1} \otimes \cdots \otimes a_{n} \in \mathcal{A}_{k_{1}}^{\circ} \otimes_{\mathcal{B}} \cdots \otimes_{\mathcal{B}} \mathcal{A}_{k_{n}}^{\circ}$. It is well-known that $\Psi$ is positive (see, e.g., [12, Theorem 3.5.6]). On the other hand, it follows from [2, Theorem 3.1] that $\Phi$ is also positive, which is the main motivation for our setting.

With Definition 3.4 and Construction 3.6 complete, we can define the notion of conditionally bi-free independence with amalgamation as follows.

Definition 3.8. Let $(\mathcal{A}, \mathbb{E}, \mathbb{F}, \varepsilon)$ be a $\mathcal{B}$ - $\mathcal{B}$-non-commutative probability space with a pair of $(\mathcal{B}, \mathcal{D})$-valued expectations. A pair of $\mathcal{B}$-algebras in $(\mathcal{A}, \mathbb{E}, \mathbb{F}, \varepsilon)$ is a pair $\left(\mathcal{C}_{\ell}, \mathcal{C}_{r}\right)$ of unital subalgebras of $\mathcal{A}$ such that

$$
\varepsilon(\mathcal{B} \otimes 1) \subset \mathcal{C}_{\ell} \subset \mathcal{A}_{\ell} \quad \text { and } \quad \varepsilon\left(1 \otimes \mathcal{B}^{\mathrm{op}}\right) \subset \mathcal{C}_{r} \subset \mathcal{A}_{r} .
$$

A family $\left\{\left(\mathcal{A}_{k, \ell}, \mathcal{A}_{k, r}\right)\right\}_{k \in K}$ of pairs of $\mathcal{B}$-algebras in $(\mathcal{A}, \mathbb{E}, \mathbb{F}, \varepsilon)$ is said to be conditionally bi-free with amalgamation over $(\mathcal{B}, \mathcal{D})$ (or $c$-bi-free over $(\mathcal{B}, \mathcal{D})$ for short) if there is a family of $\mathcal{B}$ - $\mathcal{B}$-bimodules with pairs of specified $(\mathcal{B}, \mathcal{D})$-valued states $\left\{\left(\mathcal{X}_{k}, \mathcal{X}_{k}^{\circ}, \mathfrak{p}_{k}, \mathfrak{q}_{k}\right)\right\}_{k \in K}$ and unital homomorphisms

$$
\ell_{k}: \mathcal{A}_{k, \ell} \rightarrow \mathcal{L}_{\ell}\left(\mathcal{X}_{k}\right) \text { and } r_{k}: \mathcal{A}_{k, r} \rightarrow \mathcal{L}_{r}\left(\mathcal{X}_{k}\right)
$$

such that the joint distribution of $\left\{\left(\mathcal{A}_{k, \ell}, \mathcal{A}_{k, r}\right)\right\}_{k \in K}$ with respect to $(\mathbb{E}, \mathbb{F})$ is equal to the joint distribution of the family

$$
\left\{\left(\lambda_{k} \circ \ell_{k}\left(\mathcal{A}_{k, \ell}\right), \rho_{k} \circ r_{k}\left(\mathcal{A}_{k, r}\right)\right)\right\}_{k \in K}
$$

in $\mathcal{L}(\mathcal{X})$ with respect to $\left(\mathbb{E}_{\mathcal{L}(\mathcal{X})}, \mathbb{F}_{\mathcal{L}(\mathcal{X})}\right)$, where $\left(\mathcal{X}, \mathcal{X}^{\circ}, \mathfrak{p}, \mathfrak{q}\right)=\left(*_{\mathcal{B}}\right)_{k \in K}\left(\mathcal{X}_{k}, \mathcal{X}_{k}^{\circ}, \mathfrak{p}_{k}, \mathfrak{q}_{k}\right)$.

It will be an immediate consequence of Theorem 5.5 below that the above definition does not depend on a specific choice of representations. Moreover, it follows immediately from the definition that if $\left\{\left(\mathcal{A}_{k, \ell}, \mathcal{A}_{k, r}\right)\right\}_{k \in K}$ is c-bi-free over $(\mathcal{B}, \mathcal{D})$, then the family $\left\{\mathcal{A}_{k, \ell}\right\}_{k \in K}$ (respectively $\left.\left\{\mathcal{A}_{k, r}\right\}_{k \in K}\right)$ of left $\mathcal{B}$-algebras (respectively right $\mathcal{B}$-algebras) is c-free over $(\mathcal{B}, \mathcal{D})$.

\section{Operator-VAlued CONDITIONALly BI-FREE PAIRS OF FUNCTIONS}

In order to study operator-valued conditional bi-free independence we must extend the notion of operatorvalued bi-multiplicative functions to pairs of functions.

4.1. Operator-valued conditionally bi-multiplicative pairs of functions. We begin with an observation, which will be useful later.

Remark 4.1. If $\pi \in \mathcal{B N C}(\chi)$ is a bi-non-crossing partition for some $\chi:\{1, \ldots, n\} \rightarrow\{\ell, r\}$, then there exists a unique partition $V_{1}, \ldots, V_{m}$ of $\{1, \ldots, n\}$ into $\chi$-intervals such that each $V_{k}$ a union of blocks of $\pi$ and such that $\min _{\prec_{\chi}}\left(V_{k}\right)$ and $\max _{\prec_{\chi}}\left(V_{k}\right)$ are in the same block of $\pi$ for each $k \in\{1, \ldots, m\}$. Furthermore, by reordering if necessary, we may assume $\max _{\prec_{\chi}}\left(V_{k}\right) \prec_{\chi} \min _{\prec_{\chi}}\left(V_{k+1}\right)$ for all $k$. For example, if $\pi$ has the 
following bi-non-crossing diagram

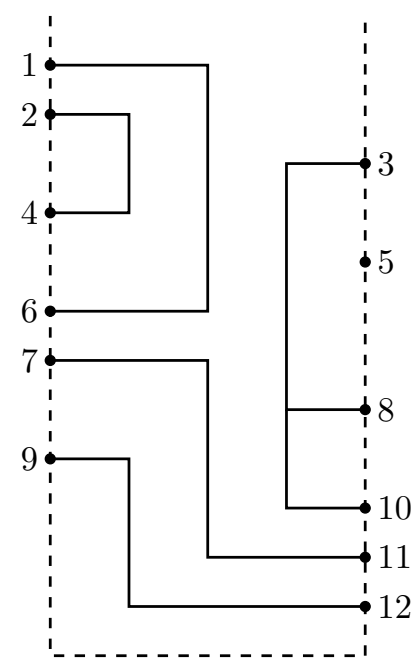

then $V_{1}=\{\{1,6\},\{2,4\}\}, V_{2}=\{\{7,11\},\{9,12\}\}$, and $V_{3}=\{\{3,8,10\},\{5\}\}$ where $\min \prec_{\chi}\left(V_{1}\right)=1$, $\max _{\prec \chi}\left(V_{1}\right)=6, \min _{\prec \chi}\left(V_{2}\right)=7, \max _{\prec_{\chi}}\left(V_{2}\right)=11, \min _{\prec \chi}\left(V_{3}\right)=10$, and $\max _{\prec \chi}\left(V_{3}\right)=3$. Note that the blocks $V_{k}^{\prime} \subset V_{k}$ containing $\min _{\prec \chi}\left(V_{k}\right)$ and $\max _{\prec \chi}\left(V_{k}\right)$ are the exterior blocks of $\pi$.

Definition 4.2. Let $(\mathcal{A}, \mathbb{E}, \mathbb{F}, \varepsilon)$ be a $\mathcal{B}$ - $\mathcal{B}$-non-commutative probability space with a pair of $(\mathcal{B}, \mathcal{D})$-valued expectations, and let

$$
\Psi: \bigcup_{n \geq 1} \bigcup_{\chi:\{1, \ldots, n\} \rightarrow\{\ell, r\}} \mathcal{B N C}(\chi) \times \mathcal{A}_{\chi(1)} \times \cdots \times \mathcal{A}_{\chi(n)} \rightarrow \mathcal{B}
$$

and

$$
\Phi: \bigcup_{n \geq 1} \bigcup_{\chi:\{1, \ldots, n\} \rightarrow\{\ell, r\}} \mathcal{B N C}(\chi) \times \mathcal{A}_{\chi(1)} \times \cdots \times \mathcal{A}_{\chi(n)} \rightarrow \mathcal{D}
$$

be a pair of functions that are linear in each $\mathcal{A}_{\chi(k)}$. It is said that $(\Psi, \Phi)$ is an operator-valued conditionally bi-multiplicative pair if for every $\chi:\{1, \ldots, n\} \rightarrow\{\ell, r\}, Z_{k} \in \mathcal{A}_{\chi(k)}, b \in \mathcal{B}$, and $\pi \in \mathcal{B N C}(\chi), \Psi$ satisfies conditions (1) to (4) of Definition 2.5 (i.e., $\Psi$ is operator-valued bi-multiplicative), and $\Phi$ satisfies conditions (1) to (3) of Definition 2.5 and the following modification of condition (4): Under the same notation with the additional assumption that $\min _{\prec_{\chi}}(\{1, \ldots, n\})$ and $\max _{\prec_{\chi}}(\{1, \ldots, n\})$ are in the same block of $\pi$, we have

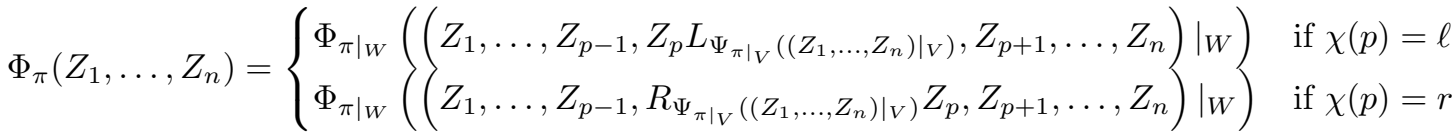

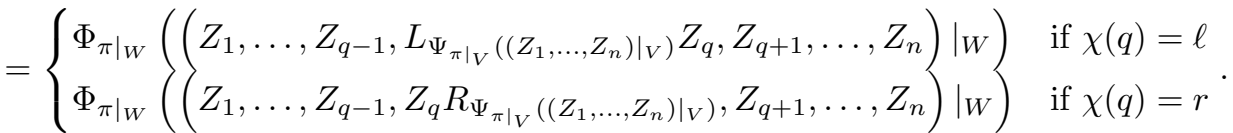

Note the additional assumption that $\min _{\prec_{\chi}}(\{1, \ldots, n\})$ and $\max _{\prec_{\chi}}(\{1, \ldots, n\})$ are in the same block of $\pi$ guarantees that $W$ contains an exterior block of $\pi$ and $V$ is a union of interior blocks of $\pi$.

Example 4.3. As with operator-valued bi-multiplicative functions, one may reduce $\Phi_{\pi}\left(Z_{1}, \ldots, Z_{n}\right)$ to an expression involving $\Psi_{1_{\chi}}$ and $\Phi_{1_{\chi}}$ for various $\chi:\{1, \ldots, m\} \rightarrow\{\ell, r\}$. For example, if $\pi$ is the bi-non-crossing partition from Remark 4.1 and $Z_{k} \in \mathcal{A}_{\chi(k)}$, then

$$
\Phi_{\pi}\left(Z_{1}, \ldots, Z_{12}\right)=\Phi_{\left.\pi\right|_{V_{1}}}\left(Z_{1}, Z_{2}, Z_{4}, Z_{6}\right) \Phi_{\pi_{V_{2}}}\left(Z_{7}, Z_{9}, Z_{11}, Z_{12}\right) \Phi_{\left.\pi\right|_{V_{3}}}\left(Z_{3}, Z_{5}, Z_{8}, Z_{10}\right)
$$

by condition (3) of Definition 2.5, which can be further reduced to

$$
\Phi_{1_{2,0}}\left(Z_{1} L_{\Psi_{1_{2,0}}\left(Z_{2}, Z_{4}\right)}, Z_{6}\right) \Phi_{1_{1,1}}\left(Z_{7} L_{\Psi_{1_{1,1}}\left(Z_{9}, Z_{12}\right)}, Z_{11}\right) \Phi_{1_{0,3}}\left(Z_{3}, R_{\Psi_{1_{0,1}}\left(Z_{5}\right)} Z_{8}, Z_{10}\right)
$$

by the modified condition (4) of Definition 4.2 . 
4.2. Operator-valued conditionally bi-free moment pairs. In this subsection, we define the operatorvalued conditionally bi-free moment pair $(\mathcal{E}, \mathcal{F})$ and show that it is operator-valued conditionally bi-multiplicative.

Definition 4.4. Let $(\mathcal{A}, \mathbb{E}, \mathbb{F}, \varepsilon)$ be a $\mathcal{B}$ - $\mathcal{B}$-non-commutative probability space with a pair of $(\mathcal{B}, \mathcal{D})$-valued expectations. The operator-valued conditionally bi-free moment pair on $\mathcal{A}$ is the pair of functions

$$
\mathcal{E}: \bigcup_{n \geq 1} \bigcup_{\chi:\{1, \ldots, n\} \rightarrow\{\ell, r\}} \mathcal{B N C}(\chi) \times \mathcal{A}_{\chi(1)} \times \cdots \times \mathcal{A}_{\chi(n)} \rightarrow \mathcal{B}
$$

and

$$
\mathcal{F}: \bigcup_{n \geq 1} \bigcup_{\chi:\{1, \ldots, n\} \rightarrow\{\ell, r\}} \mathcal{B N C}(\chi) \times \mathcal{A}_{\chi(1)} \times \cdots \times \mathcal{A}_{\chi(n)} \rightarrow \mathcal{D}
$$

where $\mathcal{E}$ is the operator-valued bi-free moment function on $\mathcal{A}$ and $\mathcal{F}_{\pi}\left(Z_{1}, \ldots, Z_{n}\right)$ for $\chi:\{1, \ldots, n\} \rightarrow\{\ell, r\}$, $\pi \in \mathcal{B N C}(\chi)$, and $Z_{k} \in \mathcal{A}_{\chi(k)}$ is defined as follows.

(1) If $\pi$ contains exactly one block (that is, $\left.\pi=1_{\chi}\right)$, define $\mathcal{F}_{1_{\chi}}\left(Z_{1}, \ldots, Z_{n}\right)=\mathbb{F}\left(Z_{1} \cdots Z_{n}\right)$.

(2) If $V_{1}, \ldots, V_{n}$ are the blocks of $\pi$, each $V_{k}$ is a $\chi$-intervals (thus all exterior), and $\max _{\prec \chi}\left(V_{k}\right) \prec_{\chi}$ $\min _{\prec \chi}\left(V_{k+1}\right)$ for all $k$, define

$$
\mathcal{F}_{\pi}\left(Z_{1}, \ldots, Z_{n}\right)=\mathcal{F}_{\left.\pi\right|_{V_{1}}}\left(\left.\left(Z_{1}, \ldots, Z_{n}\right)\right|_{V_{1}}\right) \cdots \mathcal{F}_{\left.\pi\right|_{V_{m}}}\left(\left.\left(Z_{1}, \ldots, Z_{n}\right)\right|_{V_{m}}\right)
$$

and apply step (3) to each piece.

(3) Apply a similar recursive process as in Definition 2.6 to the interior blocks of $\pi$ as follows: Let $V$ be the interior block of $\pi$ that terminates closest to the bottom. Then

- If $V=\{k+1, \ldots, n\}$ for some $k \in\{1, \ldots, n-1\}$, then $\min (V)$ is not adjacent to any spine of $\pi$ and define

$$
\mathcal{F}_{\pi}\left(Z_{1}, \ldots, Z_{n}\right)=\left\{\begin{array}{ll}
\mathcal{F}_{\pi \mid}\left(Z_{1}, \ldots, Z_{k} L_{\mathcal{E}_{\left.\pi\right|_{V}}\left(Z_{k+1}, \ldots, Z_{n}\right)}\right) & \text { if } \chi(\min (V))=\ell \\
\mathcal{F}_{\left.\pi\right|_{V} \mathrm{C}}\left(Z_{1}, \ldots, Z_{k} R_{\mathcal{E}_{\left.\pi\right|_{V}}\left(Z_{k+1}, \ldots, Z_{n}\right)}\right) & \text { if } \chi(\min (V))=r
\end{array} .\right.
$$

- Otherwise, $\min (V)$ is adjacent to a spine. Let $W$ denote the block of $\pi$ corresponding to the spine adjacent to $\min (V)$ and let $k$ be the smallest element of $W$ that is larger than $\min (V)$. Define

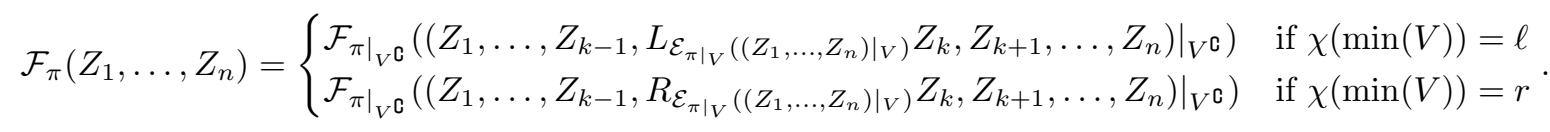

Example 4.5. Again, let $\pi$ be the bi-non-crossing partition from Remark 4.1 and $Z_{k} \in \mathcal{A}_{\chi(k)}$. Then

$$
\mathcal{F}_{\pi}\left(Z_{1}, \ldots, Z_{12}\right)=\mathbb{F}\left(Z_{1} L_{\mathbb{E}\left(Z_{2} Z_{4}\right)} Z_{6}\right) \mathbb{F}\left(Z_{7} L_{\mathbb{E}\left(Z_{9} Z_{12}\right)} Z_{11}\right) \mathbb{F}\left(Z_{3} R_{\mathbb{E}\left(Z_{5}\right)} Z_{8} Z_{10}\right) .
$$

In general, the rule is 'one uses $\mathcal{E}$ to reduce the interior blocks and then factors $\mathcal{F}_{\pi}$ according to the remaining exterior blocks.'

Theorem 4.6. Let $(\mathcal{A}, \mathbb{E}, \mathbb{F}, \varepsilon)$ be a $\mathcal{B}$ - $\mathcal{B}$-non-commutative probability space with a pair of $(\mathcal{B}, \mathcal{D})$-valued expectations. The operator-valued conditionally bi-free moment pair $(\mathcal{E}, \mathcal{F})$ on $\mathcal{A}$ is operator-valued conditionally bi-multiplicative.

Proof. The fact that the operator-valued bi-free moment function $\mathcal{E}$ on $\mathcal{A}$ is operator-valued bi-multiplicative is the main result of [4, Section 5]. It is also clear that the function $\mathcal{F}$ satisfies conditions (1), (2), and (3) of Definition 2.5.

To demonstrate the modified condition (4) in Definition 4.2, the proof relies on the techniques observed in [4. Subsection 5.3] which show that the function $\mathcal{E}$ satisfies condition (4) of Definition 2.5. In particular, we refer the reader to the proofs of [4, Lemmata 5.3.1 to 5.3.4] for additional details in that which follows. Under the same assumptions and notation, first note that the special case of the assertion holds under the additional assumption of [4, Lemma 5.3.1]; that is, there exists a block $W_{0} \subset W$ of $\pi$ such that

$$
p, q, \min _{\prec \chi}(\{1, \ldots, n\}), \max _{\prec_{\chi}}(\{1, \ldots, n\}) \in W_{0} .
$$

Indeed, suppose $\chi(p)=\ell$ (the other case is similar), and note that $W_{0}$ is the only exterior block of $\pi$. By the same arguments as in the proof of [4. Lemma 5.3.1], we have

$$
\mathcal{F}_{\pi}\left(Z_{1}, \ldots, Z_{n}\right)=\mathcal{F}_{\left.\pi\right|_{W}}\left(\left.\left(Z_{1}, \ldots, Z_{p-1}, Z_{p} L_{\mathcal{E}_{\left.\pi\right|_{V}}}\left(\left.\left(Z_{1}, \ldots, Z_{n}\right)\right|_{V}\right), Z_{p+1}, \ldots, Z_{n}\right)\right|_{W}\right)
$$


for all three possible cases, i.e., $\chi(q)=\ell ; \chi(q)=r$ and $p<q ; \chi(q)=r$ and $p>q$.

To verify the modified condition (4) in full generality, we examine the proof of [4, Lemma 5.3.4]. Suppose $\chi(p)=\ell$ (the other case is similar), and note that under the additional assumption of the modified condition (4) that there exists a block $W_{0} \subset W$ of $\pi$ such that $\min _{\prec \chi}(\{1, \ldots, n\}), \max _{\prec \chi}(\{1, \ldots, n\}) \in W_{0}$, the block $W_{0}$ is always the only exterior block of $\pi$. Let

$$
\alpha=\max _{\prec_{\chi}}\left(\left\{k \in W_{0} \mid k \preceq_{\chi} p\right\}\right), \quad \beta=\min _{\prec_{\chi}}\left(\left\{k \in W_{0} \mid q \preceq_{\chi} k\right\}\right),
$$

and let $U=\left\{k \mid \alpha \prec_{\chi} k \prec_{\chi} \beta\right\}$. Thus $U$ is a union of blocks of $\pi$. Let $\overline{W_{0}}=U^{\complement}$. Then, by the special case above (with $U$ being the $\chi$-interval), we have

$$
\mathcal{F}_{\pi}\left(Z_{1}, \ldots, Z_{n}\right)=\mathcal{F}_{\pi \mid \overline{W_{0}}}\left(\left.\left(Z_{1}, \ldots, Z_{\alpha-1}, Z_{\alpha} L_{\mathcal{E}_{\pi \mid U}\left(\left.\left(Z_{1}, \ldots, Z_{n}\right)\right|_{U}\right)}, Z_{\alpha+1}, \ldots, Z_{n}\right)\right|_{\overline{W_{0}}}\right) .
$$

Since $\mathcal{E}$ is operator-valued bi-multiplicative, we have

$$
Z_{\alpha} L_{\mathcal{E}_{\left.\pi\right|_{U}}}\left(\left.\left(Z_{1}, \ldots, Z_{n}\right)\right|_{U}\right)=Z_{p} L_{\mathcal{E}_{\left.\pi\right|_{V}}\left(\left.\left(Z_{1}, \ldots, Z_{n}\right)\right|_{V}\right)} L_{\mathcal{E}_{\left.\pi\right|_{U} \backslash V}}\left(\left.\left(Z_{1}, \ldots, Z_{n}\right)\right|_{U \backslash V}\right)
$$

if $\alpha=p$, and

$$
\mathcal{E}_{\left.\pi\right|_{U}}\left(\left.\left(Z_{1}, \ldots, Z_{n}\right)\right|_{U}\right)=\mathcal{E}_{\left.\pi\right|_{U \backslash V}}\left(\left.\left(Z_{1}, \ldots, Z_{p-1}, Z_{p} L_{\mathcal{E}_{\left.\pi\right|_{V}}\left(\left.\left(Z_{1}, \ldots, Z_{n}\right)\right|_{V}\right)}, Z_{p+1}, \ldots, Z_{n}\right)\right|_{U \backslash V}\right)
$$

otherwise. Since $W=\overline{W_{0}} \cup(U \backslash V)$, the assertion follows from applying the special case above in the opposite direction.

4.3. Operator-valued conditionally bi-free cumulant pairs. In this subsection, we recursively define the operator-valued conditionally bi-free cumulant pair $(\kappa, \mathcal{K})$ using the pair $(\mathcal{E}, \mathcal{F})$ from the previous subsection and show that it is also operator-valued conditionally bi-multiplicative.

Definition 4.7. Let $(\mathcal{A}, \mathbb{E}, \mathbb{F}, \varepsilon)$ be a $\mathcal{B}$ - $\mathcal{B}$-non-commutative probability space with a pair of $(\mathcal{B}, \mathcal{D})$-valued expectations and let $(\mathcal{E}, \mathcal{F})$ be the operator-valued conditionally bi-free moment pair on $\mathcal{A}$. The operatorvalued conditionally bi-free cumulant pair on $\mathcal{A}$ is the pair of functions

$$
\kappa: \bigcup_{n \geq 1} \bigcup_{\chi:\{1, \ldots, n\} \rightarrow\{\ell, r\}} \mathcal{B N C}(\chi) \times \mathcal{A}_{\chi(1)} \times \cdots \times \mathcal{A}_{\chi(n)} \rightarrow \mathcal{B}
$$

and

$$
\mathcal{K}: \bigcup_{n \geq 1} \bigcup_{\chi:\{1, \ldots, n\} \rightarrow\{\ell, r\}} \mathcal{B N C}(\chi) \times \mathcal{A}_{\chi(1)} \times \cdots \times \mathcal{A}_{\chi(n)} \rightarrow \mathcal{D}
$$

where $\kappa$ is the operator-valued bi-free cumulant function on $\mathcal{A}$ and $\mathcal{K}$ is recursively defined as follows.

(1) If $n=1$, then $\mathcal{K}_{1_{1,0}}\left(Z_{\ell}\right)=\mathcal{F}_{1_{1,0}}\left(Z_{\ell}\right)$ for $Z_{\ell} \in \mathcal{A}_{\ell}$ and $\mathcal{K}_{1_{0,1}}\left(Z_{r}\right)=\mathcal{F}_{1_{0,1}}\left(Z_{r}\right)$ for $Z_{r} \in \mathcal{A}_{r}$.

(2) Fix $n \geq 2, \chi:\{1, \ldots, n\} \rightarrow\{\ell, r\}, \pi \in \mathcal{B N C}(\chi)$, and $Z_{k} \in \mathcal{A}_{\chi(k)}$. If $\pi \neq 1_{\chi}$, then let $V_{1}, \ldots, V_{m}$ be the partition of $\pi$ as described in Remark 4.1. We define

$$
\mathcal{K}_{\pi}\left(Z_{1}, \ldots, Z_{n}\right)=\mathcal{K}_{\left.\pi\right|_{V_{1}}}\left(\left.\left(Z_{1}, \ldots, Z_{n}\right)\right|_{V_{1}}\right) \cdots \mathcal{K}_{\left.\pi\right|_{V_{m}}}\left(\left.\left(Z_{1}, \ldots, Z_{n}\right)\right|_{V_{m}}\right),
$$

where each $\mathcal{K}_{\left.\pi\right|_{V_{k}}}\left(\left.\left(Z_{1}, \ldots, Z_{n}\right)\right|_{V_{k}}\right)$ is defined as follows. Let $V_{k}^{\prime} \subset V_{k}$ be the block containing $\min _{\prec_{\chi}}\left(V_{k}\right)$ and $\max _{\prec_{\chi}}\left(V_{k}\right)$, let $V \subset V_{k} \backslash V_{k}^{\prime}$ be the block which terminates closest to the bottom (compared to other blocks of $V_{k}$ ). If $p=\max _{\prec_{\chi}}\left(\left\{j \in V_{k} \mid j \prec_{\chi} \min _{\prec_{\chi}}(V)\right\}\right)$ define

$$
\begin{aligned}
& \mathcal{K}_{\left.\pi\right|_{V_{k}}}\left(\left.\left(Z_{1}, \ldots, Z_{n}\right)\right|_{V_{k}}\right)
\end{aligned}
$$

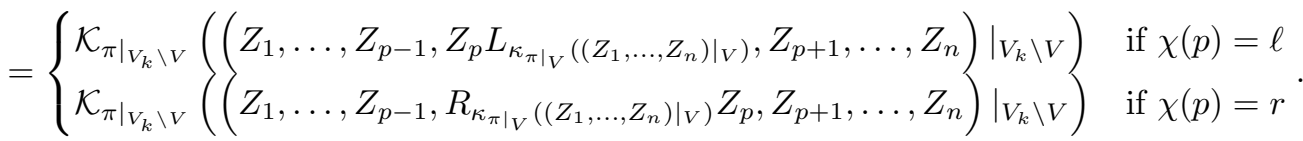

Repeat this process until the only remaining block of $V_{k}$ is $V_{k}^{\prime}$.

(3) Otherwise $\pi=1_{\chi}$ and define

$$
\mathcal{K}_{1_{\chi}}\left(Z_{1}, \ldots, Z_{n}\right)=\mathcal{F}_{1_{\chi}}\left(Z_{1}, \ldots, Z_{n}\right)-\sum_{\substack{\pi \in \mathcal{B N C} \mathcal{C}(\chi) \\ \pi \neq 1_{\chi}}} \mathcal{K}_{\pi}\left(Z_{1}, \ldots, Z_{n}\right) .
$$


Theorem 4.8. Let $(\mathcal{A}, \mathbb{E}, \mathbb{F}, \varepsilon)$ be a $\mathcal{B}$ - $\mathcal{B}$-non-commutative probability space with a pair of $(\mathcal{B}, \mathcal{D})$-valued expectations. The operator-valued conditionally bi-free cumulant pair $(\kappa, \mathcal{K})$ on $\mathcal{A}$ is operator-valued conditionally bi-multiplicative.

Proof. The fact that the operator-valued bi-free cumulant function $\kappa$ on $\mathcal{A}$ is operator-valued bi-multiplicative was proved in [4, Section 6]. Moreover, it is easy to see that the function $\mathcal{K}$ satisfies condition (3) of Definition 2.5 .

For condition (1) of Definition 2.5 we will proceed by induction on $n$ to show that condition (1) holds in greater generality. To be specific, we will demonstrate that condition (1) holds whenever $1_{\chi}$ is replaced with $\pi$. To proceed, note the base case where $n=1$ is trivial. For the inductive step, suppose the assertion holds for all $1 \leq n_{0} \leq n-1, \chi_{0}:\left\{1, \ldots, n_{0}\right\} \rightarrow\{\ell, r\}$, and $\pi_{0} \in \mathcal{B N C C}\left(\chi_{0}\right)$. Suppose $\chi:\{1, \ldots, n\} \rightarrow\{\ell, r\}$ and that $\chi(n)=\ell$ (as the other case is similar). If $q=-\infty$, then $\chi:\{1, \ldots, n\} \rightarrow\{\ell\}$ is the constant map, and thus for each $\pi \in \mathcal{B N C}(\chi), n$ necessarily belongs to an exterior block of $\pi$. Since $\mathcal{K}_{\pi}\left(Z_{1}, \ldots, Z_{n-1}, Z_{n} L_{b}\right)$ factors according to the exterior blocks of $\pi$, we have $\mathcal{K}_{\pi}\left(Z_{1}, \ldots, Z_{n-1}, Z_{n} L_{b}\right)=\mathcal{K}_{\pi}\left(Z_{1}, \ldots, Z_{n}\right) b$ if $\pi \neq 1_{\chi}$ by the induction hypothesis. Thus

$$
\begin{aligned}
\mathcal{K}_{1_{\chi}}\left(Z_{1}, \ldots, Z_{n-1}, Z_{n} L_{b}\right) & =\mathcal{F}_{1_{\chi}}\left(Z_{1}, \ldots, Z_{n-1}, Z_{n} L_{b}\right)-\sum_{\substack{\pi \in \mathcal{B N \mathcal { C }}(\chi) \\
\pi \neq 1 \chi}} \mathcal{K}_{\pi}\left(Z_{1}, \ldots, Z_{n-1}, Z_{n} L_{b}\right) \\
& =\mathcal{F}_{1_{\chi}}\left(Z_{1}, \ldots, Z_{n}\right) b-\sum_{\substack{\pi \in \mathcal{B N \mathcal { C }}(\chi) \\
\pi \neq 1_{\chi}}} \mathcal{K}_{\pi}\left(Z_{1}, \ldots, Z_{n}\right) b \\
& =\mathcal{K}_{1_{\chi}}\left(Z_{1}, \ldots, Z_{n}\right) b .
\end{aligned}
$$

If $q \neq-\infty$ and $\pi \in \mathcal{B N C}(\chi)$ such that $\pi \neq 1_{\chi}$, then as $n$ and $q$ are adjacent with respect to $\prec_{\chi}$, we have the following possible cases:

(i) $n, q \in V$ such that $V$ is an interior block of $\pi$;

(ii) $n, q \in V$ such that $V$ is an exterior block of $\pi$;

(iii) $n \in V_{1}$ and $q \in V_{2}$ such that $n=\max _{\prec_{\chi}}\left(V_{1}\right) \prec_{\chi} \min _{\prec_{\chi}}\left(V_{2}\right)=q$, and both of $V_{1}$ and $V_{2}$ are interior blocks of $\pi$;

(iv) $n \in V_{1}$ and $q \in V_{2}$ such that $n=\max _{\prec_{\chi}}\left(V_{1}\right) \prec_{\chi} \min _{\prec_{\chi}}\left(V_{2}\right)=q$, and both of $V_{1}$ and $V_{2}$ are exterior blocks of $\pi$;

(v) $n \in V_{1}$ and $q \in V_{2}$ such that $\min _{\prec_{\chi}}\left(V_{2}\right) \prec_{\chi} \min _{\prec_{\chi}}\left(V_{1}\right)$ (thus $V_{1}$ is interior with respect to $V_{2}$ ), and $V_{2}$ is an interior block of $\pi$;

(vi) $n \in V_{1}$ and $q \in V_{2}$ such that $\min _{\prec_{\chi}}\left(V_{2}\right) \prec_{\chi} \min _{\prec_{\chi}}\left(V_{1}\right)$ (thus $V_{1}$ is interior with respect to $V_{2}$ ), and $V_{2}$ is an exterior block of $\pi$;

(vii) $n \in V_{1}$ and $q \in V_{2}$ such that $\max _{\prec \chi}\left(V_{2}\right) \prec_{\chi} \max _{\prec \chi}\left(V_{1}\right)$ (thus $V_{2}$ is interior with respect to $V_{1}$ ), and $V_{1}$ is an interior block of $\pi$;

(viii) $n \in V_{1}$ and $q \in V_{2}$ such that $\max _{\prec_{\chi}}\left(V_{2}\right) \prec_{\chi} \max _{\prec_{\chi}}\left(V_{1}\right)$ (thus $V_{2}$ is interior with respect to $V_{1}$ ), and $V_{1}$ is an exterior block of $\pi$.

Since $\pi \neq 1_{\chi}$, cases $(i),(i i),(i i i),(v),(v i),(v i i)$, and (viii) follow from the induction hypothesis and from the fact that $\kappa$ is operator-valued bi-multiplicative. For case $(i v), V_{1} \subset \chi^{-1}(\{\ell\})$ and $V_{2} \subset \chi^{-1}(\{r\})$, so the result follows from the $q=-\infty$ situation (and the proof where $\chi(n)=r$ which must be run simultaneously with induction). Therefore, we have

$$
\mathcal{K}_{\pi}\left(Z_{1}, \ldots, Z_{n-1}, Z_{n} L_{b}\right)=\mathcal{K}_{\pi}\left(Z_{1}, \ldots, Z_{q-1}, Z_{q} R_{b}, Z_{q+1}, \ldots, Z_{n}\right)
$$

for all $\pi \neq 1_{\chi}$, and hence

$$
\mathcal{K}_{1_{\chi}}\left(Z_{1}, \ldots, Z_{n-1}, Z_{n} L_{b}\right)=\mathcal{K}_{1_{\chi}}\left(Z_{1}, \ldots, Z_{q-1}, Z_{q} R_{b}, Z_{q+1}, \ldots, Z_{n}\right)
$$

by the same calculation as the $q=-\infty$ situation.

The verification for condition (2) of Definition 2.5 follows from essentially the same induction arguments and casework as above with $p$ replacing $n$. The only difference is that if $q=-\infty$, then $p$ is the smallest element with respect to $\prec_{\chi}$, and hence necessarily belongs to an exterior block of $\pi$. Note this shows that the function $\mathcal{K}$ actually satisfies the additional properties that conditions (1) and (2) of Definition [2.5 hold for all $\pi \in \mathcal{B N C}(\chi)$. 
Finally, for the modified condition (4) as given in Definition 4.2, the result follows from the extended conditions (1) and (2) of Definition 2.5 as stated above along with the recursive definition in Definition 4.4 and the fact that $\kappa$ is operator-valued bi-multiplicative.

\section{UNIVERSAL MOMENT EXPRESSIONS FOR C-BI-FREE INDEPENDENCE WITH AMALGAMATION}

In this section, we will demonstrate that a family of pairs of $\mathcal{B}$-algebras is c-bi-free over $(\mathcal{B}, \mathcal{D})$ if and only if certain operator-valued moment expressions hold. To do so, we note that the shaded diagrams from Definition 2.3 and [4. Lemma 7.1.3] will be useful.

Definition 5.1. Let $\left\{\left(\mathcal{X}_{k}, \mathcal{X}_{k}^{\circ}, \mathfrak{p}_{k}, \mathfrak{q}_{k}\right)\right\}_{k \in K}$ be a family of $\mathcal{B}$ - $\mathcal{B}$-bimodules with pairs of specified $(\mathcal{B}, \mathcal{D})$-valued states, let $\lambda_{k}$ and $\rho_{k}$ be the left and right representations of $\mathcal{L}\left(\mathcal{X}_{k}\right)$ on $\mathcal{L}(\mathcal{X})$, and let $\mathcal{X}=\left(*_{\mathcal{B}}\right)_{k \in K} \mathcal{X}_{k}$. Fix $\chi:\{1, \ldots, n\} \rightarrow\{\ell, r\}, \omega:\{1, \ldots, n\} \rightarrow K, Z_{k} \in \mathcal{L}_{\chi(k)}\left(\mathcal{X}_{\omega(k)}\right)$, and let $\mu_{k}\left(Z_{k}\right)=\lambda_{\omega(k)}\left(Z_{k}\right)$ if $\chi(k)=\ell$ and $\mu_{k}\left(Z_{k}\right)=\rho_{\omega(k)}\left(Z_{k}\right)$ if $\chi(k)=r$.

For $D \in \mathcal{L R}^{\text {lat }}(\chi, \omega)$, recursively define $\mathbb{E}_{D}\left(\mu_{1}\left(Z_{1}\right), \ldots, \mu_{n}\left(Z_{n}\right)\right)$ as follows: If $D \in \mathcal{L R}_{0}^{\text {lat }}(\chi, \omega)$, then

$$
\mathbb{E}_{D}\left(\mu_{1}\left(Z_{1}\right), \ldots, \mu_{n}\left(Z_{n}\right)\right)=\left(\mathcal{E}_{\mathcal{L}(\mathcal{X})}\right)_{\pi}\left(\mu_{1}\left(Z_{1}\right), \ldots, \mu_{n}\left(Z_{n}\right)\right) \in \mathcal{B}
$$

where $\pi \in \mathcal{B N C}(\chi)$ is the bi-non-crossing partition corresponding to $D$. If every block of $D$ has a spine reaching the top, then enumerate the blocks from left to right according to their spines as $V_{1}, \ldots, V_{m}$ with $V_{j}=\left\{k_{j, 1}<\cdots<k_{j, q_{j}}\right\}$, and set

$\mathbb{E}_{D}\left(\mu_{1}\left(Z_{1}\right), \ldots, \mu_{n}\left(Z_{n}\right)\right)=\left[\left(1-\mathfrak{p}_{\omega\left(k_{1,1}\right)}\right) Z_{k_{1,1}} \cdots Z_{k_{1, q_{1}}}(1 \oplus 0)\right] \otimes \cdots \otimes\left[\left(1-\mathfrak{p}_{\omega\left(k_{m, 1}\right)}\right) Z_{k_{m, 1}} \cdots Z_{k_{m, q_{m}}}(1 \oplus 0)\right]$, which is an element of $\mathcal{X}^{\circ}$. Otherwise, apply the recursive process using $\mathbb{E}_{\mathcal{L}(\mathcal{X})}$ as in Definition [2.6 until every block of $D$ has a spine reaching the top.

Under the above assumptions and notation, it was demonstrated in [4, Lemma 7.1.3] that

$$
\mu_{1}\left(Z_{1}\right) \cdots \mu_{n}\left(Z_{n}\right)(1 \oplus 0)=\sum_{k=0}^{n} \sum_{D \in \mathcal{L} \mathcal{R}_{k}^{\text {lat }}(\chi, \omega)}\left[\sum_{\substack{D^{\prime} \in \mathcal{L} \mathcal{R}_{k}(\chi, \omega) \\ D^{\prime} \geq \text { lat }}}(-1)^{|D|-\left|D^{\prime}\right|}\right] \mathbb{E}_{D}\left(\mu_{1}\left(Z_{1}\right), \ldots, \mu_{n}\left(Z_{n}\right)\right)
$$

and, consequently,

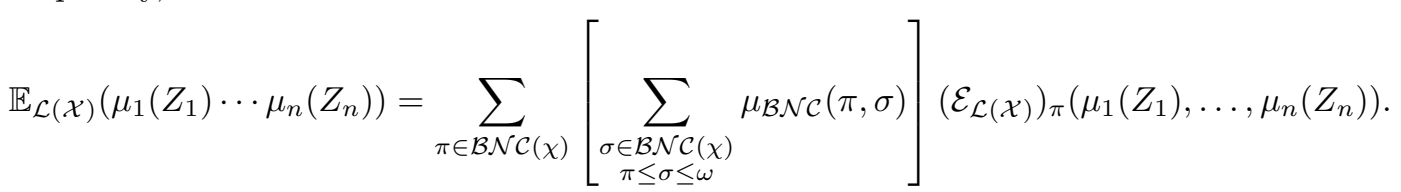

For $D \in \mathcal{L R}^{\text {latcap }}(\chi, \omega)$, we define $\mathbb{E}_{D}\left(\mu_{1}\left(Z_{1}\right), \ldots, \mu_{n}\left(Z_{n}\right)\right)$ by exactly the same recursive process that used to define $\mathbb{E}_{D^{\prime}}\left(\mu_{1}\left(Z_{1}\right), \ldots, \mu_{n}\left(Z_{n}\right)\right)$ for $D^{\prime} \in \mathcal{L} \mathcal{R}^{\text {lat }}(\chi, \omega)$. Note that, unlike $\mathbb{E}_{D^{\prime}}\left(\mu_{1}\left(Z_{1}\right), \ldots, \mu_{n}\left(Z_{n}\right)\right)$, it is not necessarily true that $\mathbb{E}_{D}\left(\mu_{1}\left(Z_{1}\right), \ldots, \mu_{n}\left(Z_{n}\right)\right) \in \mathcal{X}$ for all $D \in \mathcal{L R}^{\text {latcap }}(\chi, \omega)$ as such diagrams may have spines reaching the top which do not alternate in colour.

If $\mathbb{E}_{D}\left(\mu_{1}\left(Z_{1}\right), \ldots, \mu_{n}\left(Z_{n}\right)\right)=X_{1} \otimes \cdots \otimes X_{m}$, let

$$
\mathfrak{q}\left(\mathbb{E}_{D}\left(\mu_{1}\left(Z_{1}\right), \ldots, \mu_{n}\left(Z_{n}\right)\right)\right)=\mathfrak{q}\left(X_{1}\right) \cdots \mathfrak{q}\left(X_{m}\right) \in \mathcal{D} .
$$

Observe that although it is possible $X_{1} \otimes \cdots \otimes X_{m} \notin \mathcal{X}^{\circ}$, it is still true that every $X_{j}$ belongs to some $\mathcal{X}_{k_{j}}^{\circ}$, and thus the above expression makes sense.

Finally for $D \in \mathcal{L R}^{\text {latcap }}(\chi, \omega)$, recursively define $\mathbb{F}_{D}\left(\mu_{1}\left(Z_{1}\right), \ldots, \mu_{n}\left(Z_{n}\right)\right)$ as follows: If $D \in \mathcal{L R}_{0}^{\text {latcap }}(\chi, \omega)$, then

$$
\mathbb{F}_{D}\left(\mu_{1}\left(Z_{1}\right), \ldots, \mu_{n}\left(Z_{n}\right)\right)=\mathbb{E}_{D}\left(\mu_{1}\left(Z_{1}\right), \ldots, \mu_{n}\left(Z_{n}\right)\right) \in \mathcal{B} \subset \mathcal{D} .
$$

If every block of $D$ has a spine reaching the top, then enumerate the blocks from left to right according to their spines as $V_{1}, \ldots, V_{m}$ with $V_{j}=\left\{k_{j, 1}<\cdots<k_{j, q_{j}}\right\}$, and set

$$
\mathbb{F}_{D}\left(\mu_{1}\left(Z_{1}\right), \ldots, \mu_{n}\left(Z_{n}\right)\right)=\mathbb{F}_{\mathcal{L}(\mathcal{X})}\left(Z_{k_{1,1}} \cdots Z_{k_{1, q_{1}}}\right) \cdots \mathbb{F}_{\mathcal{L}(\mathcal{X})}\left(Z_{k_{m, 1}} \cdots Z_{k_{m, q_{m}}}\right) \in \mathcal{D} .
$$

Otherwise, apply the recursive process using $\mathbb{E}_{\mathcal{L}(\mathcal{X})}$ as in Definition 2.6 until every block of $D$ has a spine reaching the top.

Note the values of $\mathbb{F}_{D}\left(\mu_{1}\left(Z_{1}\right), \ldots, \mu_{n}\left(Z_{n}\right)\right)$ depend only on the values of $\mathbb{F}_{\mathcal{L}\left(\mathcal{X}_{k_{j}}\right)}\left(Z_{k_{j, 1}} \cdots Z_{k_{j, q_{j}}}\right)$ and the values of $\mathbb{E}_{\mathcal{L}\left(\mathcal{X}_{k_{j}}\right)}\left(Z_{k_{j, 1}} \cdots Z_{k_{j, q_{j}}}\right)$ for some $k_{j} \in K$. Hence $\mathbb{F}_{D}$ makes sense in any $\mathcal{B}$ - $\mathcal{B}$-non-commutative 
probability space with a pair of $(\mathcal{B}, \mathcal{D})$-vector expectations and will not depend on the representation of the pairs of $\mathcal{B}$-algebras.

Lemma 5.2. Under the above assumptions and notation, for all $D \in \mathcal{L R}^{\text {lat }}(\chi, \omega)$

$$
\sum_{\substack{D^{\prime} \in \mathcal{L} \mathcal{R}^{\text {latcap }} \\ D^{\prime} \leq \text { cap } D}} \mathfrak{q}\left(\mathbb{E}_{D^{\prime}}\left(\mu_{1}\left(Z_{1}\right), \ldots, \mu_{n}\left(Z_{n}\right)\right)\right)=\mathbb{F}_{D}\left(\mu_{1}\left(Z_{1}\right), \ldots, \mu_{n}\left(Z_{n}\right)\right) .
$$

Proof. If $D \in \mathcal{L R}_{0}^{\text {lat }}(\chi, \omega)$, then the only diagram $D^{\prime} \in \mathcal{L R}^{\text {latcap }}(\chi, \omega)$ such that $D^{\prime} \leq$ cap $D$ is $D$ itself. Thus the equation is trivially true by definition in this case.

For $D \in \mathcal{L R}_{m}^{\text {lat }}(\chi, \omega)$ with $0<m \leq n$, it suffices to prove the following claim: Let $V_{1}, \ldots, V_{m}$ be the blocks of $D$ with spines reaching the top, ordered from left to right according to their spines, let $V_{1}=\left\{k_{1,1}<\cdots<\right.$ $\left.k_{1, q_{1}}\right\}$, and let $V_{1,1}, \ldots, V_{1, m_{1}}$ be the blocks of $D$ which reduce to appropriate $L_{b}$ or $R_{b}$ multiplied on the left and/or right of some $Z_{k_{1, j}}$ in the recursive process. Suppose $D^{\prime}, D^{\prime \prime} \in \mathcal{L R}^{\text {latcap }}(\chi, \omega)$ are such that $D^{\prime} \leq_{\text {cap }} D, D^{\prime \prime} \leq_{\text {cap }} D$, the spine of the block $V_{1}$ reaches the top in $D^{\prime}$ but not in $D^{\prime \prime}$, and the spines of all other blocks in $D^{\prime}$ and $D^{\prime \prime}$ agree. We claim that

$$
\begin{aligned}
& \mathfrak{q}\left(\mathbb{E}_{D^{\prime}}\left(\mu_{1}\left(Z_{1}\right), \ldots, \mu_{n}\left(Z_{n}\right)\right)\right)+\mathfrak{q}\left(\mathbb{E}_{D^{\prime \prime}}\left(\mu_{1}\left(Z_{1}\right), \ldots, \mu_{n}\left(Z_{n}\right)\right)\right) \\
& \quad=\mathbb{F}_{\mathcal{L}(\mathcal{X})}\left(Z_{k_{1,1}}^{\prime} \ldots Z_{k_{1, q_{1}}}^{\prime}\right) \mathfrak{q}\left(\mathbb{E}_{D^{\prime} \backslash\left(V_{1} \cup V_{1,1} \cup \ldots \cup V_{1, m_{1}}\right)}\left(\left.\left(\mu_{1}\left(Z_{1}\right), \ldots, \mu_{n}\left(Z_{n}\right)\right)\right|_{D^{\prime} \backslash\left(V_{1} \cup V_{1,1} \cup \ldots \cup V_{1, m_{1}}\right)}\right)\right),
\end{aligned}
$$

where $Z_{k_{1, j}}^{\prime}$ is $Z_{k_{1, j}}$, potentially multiplied on the left and/or right by appropriate $L_{b}$ and $R_{b}$ such that the multiplications correspond to the blocks $V_{1,1}, \ldots, V_{1, m_{1}}$.

Indeed, if the claim is true, then for a given $D$ as above, the spine of $V_{1}$ reaches the top in exactly half of the cappings of $D$ and each such capping $D^{\prime}$ can be paired with another capping $D^{\prime \prime}$ such that the only difference between $D^{\prime}$ and $D^{\prime \prime}$ is that the spine of $V_{1}$ does not reach the top in $D^{\prime \prime}$. Adding up $\mathfrak{q}\left(\mathbb{E}_{D^{\prime}}\left(\mu_{1}\left(Z_{1}\right), \ldots, \mu_{n}\left(Z_{n}\right)\right)\right)$ and $\mathfrak{q}\left(\mathbb{E}_{D^{\prime \prime}}\left(\mu_{1}\left(Z_{1}\right), \ldots, \mu_{n}\left(Z_{n}\right)\right)\right)$ for all pairs yield the result by induction.

To prove the claim, note if $m=1$ (that is, the only spine that reaches the top is the spine of $V_{1}$ ), then $V_{1} \cup V_{1,1} \cup \cdots \cup V_{1, m_{1}}=D^{\prime}$ and we have

$$
\begin{aligned}
\mathfrak{q}\left(\mathbb{E}_{D^{\prime}}\left(\mu_{1}\left(Z_{1}\right), \ldots, \mu_{n}\left(Z_{n}\right)\right)\right) & =\mathbb{F}_{\mathcal{L}(\mathcal{X})}\left(Z_{k_{1,1}}^{\prime} \cdots Z_{k_{1, q_{1}}}^{\prime}\right)-\mathbb{E}_{\mathcal{L}(\mathcal{X})}\left(Z_{k_{1,1}}^{\prime} \cdots Z_{k_{1, q_{1}}}^{\prime}\right) \\
& =\mathbb{F}_{\mathcal{L}(\mathcal{X})}\left(Z_{k_{1,1}}^{\prime} \cdots Z_{k_{1, q_{1}}}^{\prime}\right)-\mathfrak{q}\left(\mathbb{E}_{D^{\prime \prime}}\left(\mu_{1}\left(Z_{1}\right), \ldots, \mu_{n}\left(Z_{n}\right)\right)\right)
\end{aligned}
$$

as $D^{\prime \prime}$ has no spine reaching the top and $\mathfrak{q}(b)=b$. Thus the result follows when $m=1$.

Otherwise, $m>1$. Let $V=V_{1} \cup V_{1,1} \cup \cdots \cup V_{1, m_{1}}$. Since left $\mathcal{B}$-operators commute with elements of $\mathcal{L}_{r}(\mathcal{X})$, right $\mathcal{B}$-operators commute with elements of $\mathcal{L}_{\ell}(\mathcal{X})$, and by the properties of $\mathbb{E}_{\mathcal{L}(\mathcal{X})}$ and $\mathbb{F}_{\mathcal{L}(\mathcal{X})}($ i.e., there are bi-multiplicative-like properties implied by the recursive definition), it can be checked via casework that

$$
\begin{aligned}
& \mathfrak{q}\left(\mathbb{E}_{D^{\prime}}\left(\mu_{1}\left(Z_{1}\right), \ldots, \mu_{n}\left(Z_{n}\right)\right)\right) \\
& \quad=\left(\mathbb{F}_{\mathcal{L}(\mathcal{X})}\left(Z_{k_{1,1}}^{\prime} \cdots Z_{k_{1, q_{1}}}^{\prime}\right)-\mathbb{E}_{\mathcal{L}(\mathcal{X})}\left(Z_{k_{1,1}}^{\prime} \cdots Z_{k_{1, q_{1}}}^{\prime}\right)\right) \mathfrak{q}\left(\mathbb{E}_{D^{\prime} \backslash V}\left(\left.\left(\mu_{1}\left(Z_{1}\right), \ldots, \mu_{n}\left(Z_{n}\right)\right)\right|_{D^{\prime} \backslash V}\right)\right)
\end{aligned}
$$

and

$$
\mathbb{E}_{\mathcal{L}(\mathcal{X})}\left(Z_{k_{1,1}}^{\prime} \cdots Z_{k_{1, q_{1}}}^{\prime}\right) \mathfrak{q}\left(\mathbb{E}_{D^{\prime} \backslash V}\left(\left.\left(\mu_{1}\left(Z_{1}\right), \ldots, \mu_{n}\left(Z_{n}\right)\right)\right|_{D^{\prime} \backslash V}\right)\right)=\mathfrak{q}\left(\mathbb{E}_{D^{\prime \prime}}\left(\mu_{1}\left(Z_{1}\right), \ldots, \mu_{n}\left(Z_{n}\right)\right)\right)
$$

for all $D^{\prime}$ and $D^{\prime \prime}$. Thus the claim and proof follows.

To keep track of some coefficients that occur, we make the following definition.

Definition 5.3. For $D \in \mathcal{L R}_{k}^{\text {latcap }}(\chi, \omega)$, define $C_{D}^{\prime}$ as follows: First define

$$
C_{D}=\left\{\begin{array}{ll}
\sum_{\substack{D^{\prime} \in \mathcal{L} \mathcal{R}_{k}(\chi, \omega) \\
D^{\prime} \geq \operatorname{lat} D}}(-1)^{|D|-\left|D^{\prime}\right|} & \text { if } D \in \mathcal{L} \mathcal{R}_{k}^{\text {lat }}(\chi, \omega) \\
0 & \text { otherwise }
\end{array} .\right.
$$


Recursively, starting with $k=n$, define

$$
C_{D}^{\prime}=C_{D}-\sum_{m=k+1}^{n} \sum_{\substack{D^{\prime} \in \mathcal{L} \mathcal{R}_{m}^{\text {latcap }}(\chi, \omega) \\ D^{\prime} \geq \text { cap } D}} C_{D^{\prime}}^{\prime}
$$

With Lemma 5.2 complete, we obtain the following operator-valued analogue of [6, Lemma 4.6].

Lemma 5.4. Under the above assumptions and notation,

$$
\mathbb{F}_{\mathcal{L}(\mathcal{X})}\left(\mu_{1}\left(Z_{1}\right) \cdots \mu_{n}\left(Z_{n}\right)\right)=\sum_{k=0}^{n} \sum_{D \in \mathcal{L} \mathcal{R}_{k}^{\text {latcap }}(\chi, \omega)} C_{D}^{\prime} \mathbb{F}_{D}\left(\mu_{1}\left(Z_{1}\right), \ldots, \mu_{n}\left(Z_{n}\right)\right),
$$

and

$$
C_{D}^{\prime}=\sum_{\substack{D^{\prime} \in \mathcal{L} \mathcal{R}(\chi, \omega) \\ D^{\prime} \geq \text { latcap } D}}(-1)^{|D|-\left|D^{\prime}\right|}=\sum_{m=k}^{n} \sum_{\substack{D^{\prime} \in \mathcal{L} \mathcal{R}_{m}(\chi, \omega) \\ D^{\prime} \geq \text { latcap } D}}(-1)^{|D|-\left|D^{\prime}\right|}
$$

for $D \in \mathcal{L R}_{k}^{\text {latcap }}(\chi, \omega)$.

Proof. For $Z_{1}, \ldots, Z_{n}$ as above, the expression $\mathbb{F}_{\mathcal{L}(\mathcal{X})}\left(\mu_{1}\left(Z_{1}\right) \cdots \mu_{n}\left(Z_{n}\right)\right)$ is obtained by applying $\mathfrak{q}$ to the left-hand side of equation (3). Using Definition [5.3, we have

$$
\begin{aligned}
& \mathbb{F}_{\mathcal{L}(\mathcal{X})}\left(\mu_{1}\left(Z_{1}\right) \cdots \mu_{n}\left(Z_{n}\right)\right) \\
& =\mathfrak{q}\left(\mu_{1}\left(Z_{1}\right) \cdots \mu_{n}\left(Z_{n}\right)(1 \oplus 0)\right) \\
& =\sum_{k=0}^{n} \sum_{D \in \mathcal{L} \mathcal{R}_{k}^{\text {lat }}(\chi, \omega)} C_{D} \mathfrak{q}\left(\mathbb{E}_{D}\left(\mu_{1}\left(Z_{1}\right), \ldots, \mu_{n}\left(Z_{n}\right)\right)\right) \\
& =\sum_{k=0}^{n} \sum_{D \in \mathcal{L} \mathcal{R}_{k}^{\text {lat }}(\chi, \omega)} C_{D}\left(\mathbb{F}_{D}\left(\mu_{1}\left(Z_{1}\right), \ldots, \mu_{n}\left(Z_{n}\right)\right)-\sum_{\substack{D^{\prime} \in \mathcal{L} \mathcal{R}^{\text {latcap }} \\
D^{\prime} \leq \text { cap } D \\
D^{\prime} \neq D}} \mathfrak{q}\left(\mathbb{E}_{D^{\prime}}\left(\mu_{1}\left(Z_{1}\right), \ldots, \mu_{n}\left(Z_{n}\right)\right)\right)\right) \\
& =\sum_{k=0}^{n} \sum_{D \in \mathcal{L} \mathcal{R}_{k}^{\text {latcap }}(\chi, \omega)} C_{D}^{\prime} \mathbb{F}_{D}\left(\mu_{1}\left(Z_{1}\right), \ldots, \mu_{n}\left(Z_{n}\right)\right),
\end{aligned}
$$

where the third equality follows from Lemma 5.2 and the fourth equality follows from Definition 5.3 as the coefficient $C_{D}^{\prime}$ for $D \in \mathcal{L R}_{k}^{\text {latcap }}(\chi, \omega)$ was specifically defined this way. The second result regarding $C_{D}^{\prime}$ is exactly the content of [6, Lemma 4.7].

Combining these results, we have the following moment type characterization of c-bi-free independence with amalgamation.

Theorem 5.5. A family $\left\{\left(\mathcal{A}_{k, \ell}, \mathcal{A}_{k, r}\right)\right\}_{k \in K}$ of pairs of $\mathcal{B}$-algebras in a $\mathcal{B}$ - $\mathcal{B}$-non-commutative probability space with a pair of $(\mathcal{B}, \mathcal{D})$-valued expectations $(\mathcal{A}, \mathbb{E}, \mathbb{F}, \varepsilon)$ is c-bi-free over $(\mathcal{B}, \mathcal{D})$ if and only if

$$
\mathbb{E}\left(Z_{1} \cdots Z_{n}\right)=\sum_{\pi \in \mathcal{B N \mathcal { C }}(\chi)}\left[\sum_{\substack{\sigma \in \mathcal{B N \mathcal { C }}(\chi) \\ \pi \leq \sigma \leq \omega}} \mu_{\mathcal{B N C}(\pi, \sigma)}\right] \mathcal{E}_{\pi}\left(Z_{1}, \ldots, Z_{n}\right)
$$

and

$$
\mathbb{F}\left(Z_{1} \cdots Z_{n}\right)=\sum_{D \in \mathcal{L} \mathcal{R}^{\text {latcap }}(\chi, \omega)}\left[\sum_{\substack{D^{\prime} \in \mathcal{L} \mathcal{R}(\chi, \omega) \\ D^{\prime} \geq \text { latcap } D}}(-1)^{|D|-\left|D^{\prime}\right|}\right] \mathbb{F}_{D}\left(Z_{1}, \ldots, Z_{n}\right)
$$

for all $n \geq 1, \chi:\{1, \ldots, n\} \rightarrow\{\ell, r\}, \omega:\{1, \ldots, n\} \rightarrow K$, and $Z_{1}, \ldots, Z_{n} \in \mathcal{A}$ with $Z_{k} \in \mathcal{A}_{\omega(k), \chi(k)}$. 
Proof. Under the above notation, if the family $\left\{\left(\mathcal{A}_{k, \ell}, \mathcal{A}_{k, r}\right)\right\}_{k \in K}$ is c-bi-free over $(\mathcal{B}, \mathcal{D})$, then there exists a family $\left\{\left(\mathcal{X}_{k}, \mathcal{X}_{k}^{\circ}, \mathfrak{p}_{k}, \mathfrak{q}_{k}\right)\right\}_{k \in K}$ such that

$$
\mathbb{E}\left(Z_{1} \cdots Z_{n}\right)=\mathbb{E}_{\mathcal{L}(\mathcal{X})}\left(\mu_{1}\left(Z_{1}\right) \cdots \mu_{n}\left(Z_{n}\right)\right) \quad \text { and } \quad \mathbb{F}\left(Z_{1} \cdots Z_{n}\right)=\mathbb{F}_{\mathcal{L}(\mathcal{X})}\left(\mu_{1}\left(Z_{1}\right) \cdots \mu_{n}\left(Z_{n}\right)\right),
$$

where each $Z_{k}$ on the right-hand side of the above equations is identified as $\ell_{k}\left(Z_{k}\right)$ if $\chi(k)=\ell$ and $r_{k}\left(Z_{k}\right)$ if $\chi(k)=r$ acting on $\mathcal{X}_{\omega(k)}$. The fact that equation (5) holds is part of [4, Theorem 7.1.4], and the fact that equation (6) holds follows from Lemma 5.4

Conversely, suppose equations (5) and (6) hold. By Theorem 3.5 there exist $\left(\mathcal{X}, \mathcal{X}^{\circ}, \mathfrak{p}, \mathfrak{q}\right)$ and a unital homomorphism $\theta: \mathcal{A} \rightarrow \mathcal{L}(\mathcal{X})$ such that

$$
\begin{gathered}
\theta\left(L_{b_{1}} R_{b_{2}}\right)=L_{b_{1}} R_{b_{2}}, \quad \theta\left(\mathcal{A}_{\ell}\right) \subset \mathcal{L}_{\ell}(\mathcal{X}), \quad \theta\left(\mathcal{A}_{r}\right) \subset \mathcal{L}_{r}(\mathcal{X}), \\
\mathbb{E}_{\mathcal{L}(\mathcal{X})}(\theta(Z))=\mathbb{E}(Z), \quad \text { and } \quad \mathbb{F}_{\mathcal{L}(\mathcal{X})}(\theta(Z))=\mathbb{F}(Z)
\end{gathered}
$$

for all $b_{1}, b_{2} \in \mathcal{B}$ and $Z \in \mathcal{A}$. For each $k \in K$, let $\left(\mathcal{X}_{k}, \mathcal{X}_{k}^{\circ}, \mathfrak{p}_{k}, \mathfrak{q}_{k}\right)$ be a copy of $\left(\mathcal{X}, \mathcal{X}^{\circ}, \mathfrak{p}, \mathfrak{q}\right)$, and let $\ell_{k}$ and $r_{k}$ be copies of $\theta: \mathcal{A} \rightarrow \mathcal{L}\left(\mathcal{X}_{k}\right)$. By [4, Lemma 7.1.3] and Lemma [5.4] we have

$$
\mathbb{E}\left(Z_{1} \cdots Z_{n}\right)=\mathbb{E}_{\mathcal{L}\left((* \mathcal{B})_{k \in K} \mathcal{X}_{k}\right)}\left(\mu_{1}\left(Z_{1}\right) \cdots \mu_{n}\left(Z_{n}\right)\right) \quad \text { and } \quad \mathbb{F}\left(Z_{1} \cdots Z_{n}\right)=\mathbb{F}_{\mathcal{L}\left((* \mathcal{B})_{k \in K} \mathcal{X}_{k}\right)}\left(\mu_{1}\left(Z_{1}\right) \cdots \mu_{n}\left(Z_{n}\right)\right),
$$

where each $Z_{k}$ on the right-hand side of the above equations is identified as $\theta\left(Z_{k}\right)$ acting on $\mathcal{X}_{\omega(k)}$. Hence, the family $\left\{\left(\mathcal{A}_{k, \ell}, \mathcal{A}_{k, r}\right)\right\}_{k \in K}$ is c-bi-free over $(\mathcal{B}, \mathcal{D})$ by definition.

As $\mathbb{F}_{D}\left(Z_{1}, \ldots, Z_{n}\right)$ and $\mathcal{E}_{\pi}\left(Z_{1}, \ldots, Z_{n}\right)$ depend only on the distributions of individual pairs $\left(\mathcal{A}_{k, \ell}, \mathcal{A}_{k, r}\right)$ inside our $\mathcal{B}$ - $\mathcal{B}$-non-commutative probability space with a pair of $(\mathcal{B}, \mathcal{D})$-valued expectations, we obtain that Definition 3.8 is well-defined in that the joint distributions do not depend on the representations.

\section{Additivity of OPERATOR-VAlued CONDitionally Bi-Free CUMUlant Pairs}

The goal of this section is to prove the operator-valued analogue of [6. Theorem 4.1]; namely that conditionally bi-free independence with amalgamation is equivalent to the vanishing of mixed operator-valued bi-free and conditionally bi-free cumulants. To establish the result, we will need a method, analogous to [11, Lemma 3.8] for constructing a pair of $\mathcal{B}$-algebras with any given operator-valued bi-free and conditionally bi-free cumulants. To this end, we discuss moment and cumulant series first.

Let $(\mathcal{A}, \mathbb{E}, \mathbb{F}, \varepsilon)$ be a $\mathcal{B}$ - $\mathcal{B}$-non-commutative probability space with a pair of $(\mathcal{B}, \mathcal{D})$-valued expectations, and let $\left(\mathcal{C}_{\ell}, \mathcal{C}_{r}\right)$ be a pair of $\mathcal{B}$-algebras such that

$$
\mathcal{C}_{\ell}=\operatorname{alg}\left(\left\{Z_{i}\right\}_{i \in I}, \varepsilon(\mathcal{B} \otimes 1)\right) \quad \text { and } \quad \mathcal{C}_{r}=\operatorname{alg}\left(\left\{Z_{j}\right\}_{j \in J}, \varepsilon\left(1 \otimes \mathcal{B}^{\text {op }}\right)\right)
$$

for some $\left\{Z_{i}\right\}_{i \in I} \subset \mathcal{A}_{\ell}$ and $\left\{Z_{j}\right\}_{j \in J} \subset \mathcal{A}_{r}$. By discussions in [11, Section 2] and by using the operatorvalued conditionally bi-multiplicative properties, only certain operator-valued bi-free and conditionally bifree moments/cumulants are required to study the joint distributions of elements in $\operatorname{alg}\left(\mathcal{C}_{\ell}, \mathcal{C}_{r}\right)$ with respect to $(\mathbb{E}, \mathbb{F})$. We make the following notation (in addition to [11, Notation 2.18] with some slight notational changes) and definition to describe the necessary moments and cumulants.

Notation 6.1. Let $\mathcal{Z}=\left\{Z_{i}\right\}_{i \in I} \sqcup\left\{Z_{j}\right\}_{j \in J}$ be as above, $n \geq 1, \omega:\{1, \ldots, n\} \rightarrow I \sqcup J$, and $b_{1}, \ldots, b_{n-1} \in \mathcal{B}$.

- If $\omega(k) \in I$ for all $k$, define

$$
\begin{aligned}
\nu_{\omega}^{\mathcal{Z}}\left(b_{1}, \ldots, b_{n-1}\right) & =\mathbb{E}\left(Z_{\omega(1)} L_{b_{1}} Z_{\omega(2)} \cdots L_{b_{n-1}} Z_{\omega(n)}\right) \in \mathcal{B} \\
\mu_{\omega}^{\mathcal{Z}}\left(b_{1}, \ldots, b_{n-1}\right) & =\mathbb{F}\left(Z_{\omega(1)} L_{b_{1}} Z_{\omega(2)} \cdots L_{b_{n-1}} Z_{\omega(n)}\right) \in \mathcal{D} \\
\rho_{\omega}^{\mathcal{Z}}\left(b_{1}, \ldots, b_{n-1}\right) & =\kappa_{1_{\chi_{\omega}}}\left(Z_{\omega(1)}, L_{b_{1}} Z_{\omega(2)}, \ldots, L_{b_{n-1}} Z_{\omega(n)}\right) \in \mathcal{B}, \text { and } \\
\eta_{\omega}^{\mathcal{Z}}\left(b_{1}, \ldots, b_{n-1}\right) & =\mathcal{K}_{1_{\chi_{\omega}}}\left(Z_{\omega(1)}, L_{b_{1}} Z_{\omega(2)}, \ldots, L_{b_{n-1}} Z_{\omega(n)}\right) \in \mathcal{D} .
\end{aligned}
$$

- If $\omega(k) \in J$ for all $k$, define

$$
\begin{aligned}
\nu_{\omega}^{\mathcal{Z}}\left(b_{1}, \ldots, b_{n-1}\right) & =\mathbb{E}\left(Z_{\omega(1)} R_{b_{1}} Z_{\omega(2)} \cdots R_{b_{n-1}} Z_{\omega(n)}\right) \in \mathcal{B}, \\
\mu_{\omega}^{\mathcal{Z}}\left(b_{1}, \ldots, b_{n-1}\right) & =\mathbb{F}\left(Z_{\omega(1)} R_{b_{1}} Z_{\omega(2)} \cdots R_{b_{n-1}} Z_{\omega(n)}\right) \in \mathcal{D} \\
\rho_{\omega}^{\mathcal{Z}}\left(b_{1}, \ldots, b_{n-1}\right) & =\kappa_{1_{\chi \omega}}\left(Z_{\omega(1)}, R_{b_{1}} Z_{\omega(2)}, \ldots, R_{b_{n-1}} Z_{\omega(n)}\right) \in \mathcal{B}, \text { and } \\
\eta_{\omega}^{\mathcal{Z}}\left(b_{1}, \ldots, b_{n-1}\right) & =\mathcal{K}_{1_{\chi_{\omega}}}\left(Z_{\omega(1)}, R_{b_{1}} Z_{\omega(2)}, \ldots, R_{b_{n-1}} Z_{\omega(n)}\right) \in \mathcal{D} .
\end{aligned}
$$


- Otherwise, let $k_{\ell}=\min \{k \mid \omega(k) \in I\}$ and $k_{r}=\min \{k \mid \omega(k) \in J\}$. Then $\left\{k_{\ell}, k_{r}\right\}=\left\{1, k_{0}\right\}$ for some $k_{0}$. Define $\nu_{\omega}^{\mathcal{Z}}\left(b_{1}, \ldots, b_{n-1}\right)$ and $\mu_{\omega}^{\mathcal{Z}}\left(b_{1}, \ldots, b_{n-1}\right)$ to be

$$
\mathbb{E}\left(Z_{\omega(1)} C_{b_{1}}^{\omega(2)} Z_{\omega(2)} \cdots C_{b_{k_{0}-2}}^{\omega\left(k_{0}-1\right)} Z_{\omega\left(k_{0}-1\right)} Z_{\omega\left(k_{0}\right)} C_{b_{k_{0}-1}}^{\omega\left(k_{0}+1\right)} Z_{\omega\left(k_{0}+1\right)} \cdots C_{b_{n-3}}^{\omega(n-1)} Z_{\omega(n-1)} C_{b_{n-2}}^{\omega(n)} Z_{\omega(n)} C_{b_{n-1}}^{\omega(n)}\right) \in \mathcal{B}
$$

and

$$
\mathbb{F}\left(Z_{\omega(1)} C_{b_{1}}^{\omega(2)} Z_{\omega(2)} \cdots C_{b_{k_{0}-2}}^{\omega\left(k_{0}-1\right)} Z_{\omega\left(k_{0}-1\right)} Z_{\omega\left(k_{0}\right)} C_{b_{k_{0}-1}}^{\omega\left(k_{0}+1\right)} Z_{\omega\left(k_{0}+1\right)} \cdots C_{b_{n-3}}^{\omega(n-1)} Z_{\omega(n-1)} C_{b_{n-2}}^{\omega(n)} Z_{\omega(n)} C_{b_{n-1}}^{\omega(n)}\right) \in \mathcal{D}
$$

respectively, and define $\rho_{\omega}^{\mathcal{Z}}\left(b_{1}, \ldots, b_{n-1}\right)$ and $\eta_{\omega}^{\mathcal{Z}}\left(b_{1}, \ldots, b_{n-1}\right)$ to be

$\kappa_{1_{\chi_{\omega}}}\left(Z_{\omega(1)}, C_{b_{1}}^{\omega(2)} Z_{\omega(2)}, \ldots, C_{b_{k_{0}-2}}^{\omega\left(k_{0}-1\right)} Z_{\omega\left(k_{0}-1\right)}, Z_{\omega\left(k_{0}\right)}, C_{b_{k_{0}-1}}^{\omega\left(k_{0}+1\right)} Z_{\omega\left(k_{0}+1\right)}, \ldots, C_{b_{n-3}}^{\omega(n-1)} Z_{\omega(n-1)}, C_{b_{n-2}}^{\omega(n)} Z_{\omega(n)} C_{b_{n-1}}^{\omega(n)}\right) \in \mathcal{B}$

and

$\mathcal{K}_{1_{\chi \omega}}\left(Z_{\omega(1)}, C_{b_{1}}^{\omega(2)} Z_{\omega(2)}, \ldots, C_{b_{k_{0}-2}}^{\omega\left(k_{0}-1\right)} Z_{\omega\left(k_{0}-1\right)}, Z_{\omega\left(k_{0}\right)}, C_{b_{k_{0}-1}}^{\omega\left(k_{0}+1\right)} Z_{\omega\left(k_{0}+1\right)}, \ldots, C_{b_{n-3}}^{\omega(n-1)} Z_{\omega(n-1)}, C_{b_{n-2}}^{\omega(n)} Z_{\omega(n)} C_{b_{n-1}}^{\omega(n)}\right) \in \mathcal{D}$

respectively, where

$$
C_{b}^{\omega(k)}=\left\{\begin{array}{ll}
L_{b} & \text { if } \omega(k) \in I \\
R_{b} & \text { if } \omega(k) \in J
\end{array} .\right.
$$

Definition 6.2. Let $\mathcal{Z}=\left\{Z_{i}\right\}_{i \in I} \sqcup\left\{Z_{j}\right\}_{j \in J}$ be as above. The moment and cumulant series of $\mathcal{Z}$ with respect to $(\mathbb{E}, \mathbb{F})$ are the collections of maps

$$
\begin{aligned}
\nu^{\mathcal{Z}} & =\left\{\nu_{\omega}^{\mathcal{Z}}: \mathcal{B}^{n-1} \rightarrow \mathcal{B} \mid n \geq 1, \omega:\{1, \ldots, n\} \rightarrow I \sqcup J\right\}, \\
\mu^{\mathcal{Z}} & =\left\{\mu_{\omega}^{\mathcal{Z}}: \mathcal{B}^{n-1} \rightarrow \mathcal{D} \mid n \geq 1, \omega:\{1, \ldots, n\} \rightarrow I \sqcup J\right\},
\end{aligned}
$$

and

$$
\begin{aligned}
\rho^{\mathcal{Z}} & =\left\{\rho_{\omega}^{\mathcal{Z}}: \mathcal{B}^{n-1} \rightarrow \mathcal{B} \mid n \geq 1, \omega:\{1, \ldots, n\} \rightarrow I \sqcup J\right\}, \\
\eta^{\mathcal{Z}} & =\left\{\eta_{\omega}^{\mathcal{Z}}: \mathcal{B}^{n-1} \rightarrow \mathcal{D} \mid n \geq 1, \omega:\{1, \ldots, n\} \rightarrow I \sqcup J\right\},
\end{aligned}
$$

respectively. Note that if $n=1$, then $\nu_{\omega}^{\mathcal{Z}}=\rho_{\omega}^{\mathcal{Z}}=\mathbb{E}\left(Z_{\omega(1)}\right)$ and $\mu_{\omega}^{\mathcal{Z}}=\eta_{\omega}^{\mathcal{Z}}=\mathbb{F}\left(Z_{\omega(1)}\right)$.

Lemma 6.3. Let $I$ and $J$ be non-empty disjoint index sets, and let $\mathcal{B}$ and $\mathcal{D}$ be unital algebras such that $1:=1_{\mathcal{D}} \in \mathcal{B} \subset \mathcal{D}$. For every $n \geq 1$ and $\omega:\{1, \ldots, n\} \rightarrow I \sqcup J$, let $\Theta_{\omega}: \mathcal{B}^{n-1} \rightarrow \mathcal{B}$ and $\Upsilon_{\omega}: \mathcal{B}^{n-1} \rightarrow \mathcal{D}$ be linear in each coordinate. There exist a $\mathcal{B}-\mathcal{B}$-non-commutative probability space with a pair of $(\mathcal{B}, \mathcal{D})$-valued expectations $(\mathcal{A}, \mathbb{E}, \mathbb{F}, \varepsilon)$ and elements $\left\{Z_{i}\right\}_{i \in I} \subset \mathcal{A}_{\ell}$ and $\left\{Z_{j}\right\}_{j \in J} \subset \mathcal{A}_{r}$ such that if $\mathcal{Z}=\left\{Z_{i}\right\}_{i \in I} \sqcup\left\{Z_{j}\right\}_{j \in J}$, then

$$
\rho_{\omega}^{\mathcal{Z}}\left(b_{1}, \ldots, b_{n-1}\right)=\Theta_{\omega}\left(b_{1}, \ldots, b_{n-1}\right) \quad \text { and } \quad \eta_{\omega}^{\mathcal{Z}}\left(b_{1}, \ldots, b_{n-1}\right)=\Upsilon_{\omega}\left(b_{1}, \ldots, b_{n-1}\right)
$$

for all $n \geq 1, \omega:\{1, \ldots, n\} \rightarrow I \sqcup J$, and $b_{1}, \ldots, b_{n-1} \in \mathcal{B}$.

Proof. By the same construction presented in the proof of [11, Lemma 3.8], there exist a $\mathcal{B}$ - $\mathcal{B}$-non-commutative probability space $(\mathcal{A}, \mathbb{E}, \varepsilon)$ and $\mathcal{Z}=\left\{Z_{i}\right\}_{i \in I} \sqcup\left\{Z_{j}\right\}_{j \in J}$ with $\left\{Z_{i}\right\}_{i \in I} \subset \mathcal{A}_{\ell}$ and $\left\{Z_{j}\right\}_{j \in J} \subset \mathcal{A}_{r}$ such that

$$
\rho_{\omega}^{\mathcal{Z}}\left(b_{1}, \ldots, b_{n-1}\right)=\Theta_{\omega}\left(b_{1}, \ldots, b_{n-1}\right)
$$

for all $n \geq 1, \omega:\{1, \ldots, n\} \rightarrow I \sqcup J$, and $b_{1}, \ldots, b_{n-1} \in \mathcal{B}$. Thus we need only define an expectation $\mathbb{F}$ to produce the correct operator-valued conditionally bi-free cumulants.

For $n \geq 1, \omega:\{1, \ldots, n\} \rightarrow I \sqcup J$, and $b_{1}, \ldots, b_{n+1} \in \mathcal{B}$, let

$$
C_{b}^{\omega(k)}=\left\{\begin{array}{ll}
L_{b} & \text { if } \omega(k) \in I \\
R_{b} & \text { if } \omega(k) \in J
\end{array},\right.
$$

and define

$$
\widehat{\Upsilon}_{1_{\chi \omega}}\left(C_{b_{1}}^{\omega(1)} Z_{\omega(1)}, \ldots, C_{b_{n-1}}^{\omega(n-1)} Z_{\omega(n-1)}, C_{b_{n}}^{\omega(n)} Z_{\omega(n)} C_{b_{n+1}}^{\omega(n)}\right) \in \mathcal{D}
$$

like how $\widehat{\Theta}_{1_{\chi_{\omega}}}$ is defined in the proof of [11, Lemma 3.8] using $\Upsilon_{\omega}$ instead of $\Theta_{\omega}$. Subsequently, for $\omega$ : $\{1, \ldots, n\} \rightarrow I \sqcup J$ and $\pi \in \mathcal{B N C}\left(\chi_{\omega}\right)$, define

$$
\widehat{\Upsilon}_{\pi}\left(C_{b_{1}}^{\omega(1)} Z_{\omega(1)}, \ldots, C_{b_{n-1}}^{\omega(n-1)} Z_{\omega(n-1)}, C_{b_{n}}^{\omega(n)} Z_{\omega(n)} C_{b_{n+1}}^{\omega(n)}\right) \in \mathcal{D}
$$


by selecting one of the many possible ways to reduce an operator-valued conditionally bi-multiplicative function where $\widehat{\Theta}_{1_{\chi}}$ is used for interior blocks and $\widehat{\Upsilon}_{1_{\chi}}$ is used for exterior blocks.

As seen in the proof of [11, Lemma 3.8], every element in $\mathcal{A}$ is a linear combination of the form

$$
C_{b_{1}}^{\omega(1)} Z_{\omega(1)} \cdots C_{b_{n}}^{\omega(n)} Z_{\omega(n)} L_{b} R_{b^{\prime}}+\mathcal{I}
$$

where $n \geq 0, \omega:\{1, \ldots, n\} \rightarrow I \sqcup J$ when $n \geq 1, b_{1}, \ldots, b_{n}, b, b^{\prime} \in \mathcal{B}$, and $\mathcal{I}$ is some two-sided ideal. Define $\mathbb{F}: \mathcal{A} \rightarrow \mathcal{D}$ by

$$
\mathbb{F}\left(L_{b} R_{b^{\prime}}+\mathcal{I}\right)=b b^{\prime}
$$

for all $b, b^{\prime} \in \mathcal{B}$, and

$$
\mathbb{F}\left(C_{b_{1}}^{\omega(1)} Z_{\omega(1)} \cdots C_{b_{n}}^{\omega(n)} Z_{\omega(n)} L_{b} R_{b^{\prime}}+\mathcal{I}\right)=\sum_{\pi \in \mathcal{B N \mathcal { N }}\left(\chi_{\omega}\right)} \widehat{\Upsilon}_{\pi}\left(C_{b_{1}}^{\omega(1)} Z_{\omega(1)}, \ldots, C_{b_{n-1}}^{\omega(n-1)} Z_{\omega(n-1)}, C_{b_{n}}^{\omega(n)} Z_{\omega(n)} C_{b b^{\prime}}\right)
$$

for all $n \geq 1$ and $\omega:\{1, \ldots, n\} \rightarrow I \sqcup J$, where $C_{b b^{\prime}}=L_{b b^{\prime}}$ if $\omega(n) \in I$ and $C_{b b^{\prime}}=R_{b b^{\prime}}$ if $\omega(n) \in J$, and extend $\mathbb{F}$ by linearity. By construction and commutation in $\mathcal{A}$, one can verify that $\mathbb{F}$ is well-defined and

$$
\mathbb{F}\left(L_{b} R_{b^{\prime}} Z+\mathcal{I}\right)=b \mathbb{F}(Z+\mathcal{I}) b^{\prime} \quad \text { and } \quad \mathbb{F}\left(Z L_{b}+\mathcal{I}\right)=\mathbb{F}\left(Z R_{b}+\mathcal{I}\right)
$$

for all $b, b^{\prime} \in \mathcal{B}$ and $Z+\mathcal{I} \in \mathcal{A}$. Finally, since Definition 4.7 completely determines the operator-valued conditionally bi-free cumulants and by our definition of $\hat{\Upsilon}$ via a choice of operator-valued conditionally bimultiplicative reduction, [11, Lemma 3.8] with an induction argument together imply that if $\mathcal{Z}=\left\{Z_{i}\right\}_{i \in I} \sqcup$ $\left\{Z_{j}\right\}_{j \in J}$, then

$$
\eta_{\omega}^{\mathcal{Z}}\left(b_{1}, \ldots, b_{n-1}\right)=\Upsilon_{\omega}\left(b_{1}, \ldots, b_{n-1}\right)
$$

for all $n \geq 1, \omega:\{1, \ldots, n\} \rightarrow I \sqcup J$, and $b_{1}, \ldots, b_{n-1} \in \mathcal{B}$.

We are now ready to prove the main result of this section.

Theorem 6.4. A family $\left\{\left(\mathcal{A}_{k, \ell}, \mathcal{A}_{k, r}\right)\right\}_{k \in K}$ of pairs of $\mathcal{B}$-algebras in a $\mathcal{B}$ - $\mathcal{B}$-non-commutative probability space with a pair of $(\mathcal{B}, \mathcal{D})$-valued expectations $(\mathcal{A}, \mathbb{E}, \mathbb{F}, \varepsilon)$ is c-bi-free over $(\mathcal{B}, \mathcal{D})$ if and only if for all $n \geq 2$, $\chi:\{1, \ldots, n\} \rightarrow\{\ell, r\}, \omega:\{1, \ldots, n\} \rightarrow K$, and $Z_{k} \in \mathcal{A}_{\omega(k), \chi(k)}$, we have

$$
\kappa_{1_{\chi}}\left(Z_{1}, \ldots, Z_{n}\right)=\mathcal{K}_{1_{\chi}}\left(Z_{1}, \ldots, Z_{n}\right)=0
$$

whenever $\omega$ is not constant.

Proof. If all mixed cumulants vanish, then $\left\{\left(\mathcal{A}_{k, \ell}, \mathcal{A}_{k, r}\right)\right\}_{k \in K}$ is bi-free over $\mathcal{B}$ so equation (5) holds. To see that equation (6) also holds, recall from [6, Subsection 4.2] that $\mathcal{B N C}(\chi, i e)$ denotes the set of all pairs $(\pi, \iota)$ where $\pi \in \mathcal{B N C}(\chi)$ is a bi-non-crossing partition and $\iota: \pi \rightarrow\{i, e\}$ is a function on the blocks of $\pi$. By Definitions 4.4 and 4.7, and the assumption that all mixed cumulants vanish, we have

$$
\mathbb{F}\left(Z_{1} \cdots Z_{n}\right)=\mathcal{F}_{1_{\chi}}\left(Z_{1}, \ldots, Z_{n}\right)=\sum_{\substack{\pi \in \mathcal{B N \mathcal { C }}(\chi) \\ \pi \leq \omega}} \mathcal{K}_{\pi}\left(Z_{1}, \ldots, Z_{n}\right)
$$

By applying Definition 4.7 recursively, we obtain that

$$
\mathbb{F}\left(Z_{1} \cdots Z_{n}\right)=\sum_{\substack{(\pi, \iota) \in \mathcal{B N \mathcal { C }}(\chi, i e) \\ \pi \leq \omega}} c(\chi, \omega ; \pi, \iota) \Theta_{(\pi, \iota)}\left(Z_{1}, \ldots, Z_{n}\right)
$$

where $c(\chi, \omega ; \pi, \iota) \Theta_{(\pi, \iota)}\left(Z_{1}, \ldots, Z_{n}\right)$ for $(\pi, \iota) \in \mathcal{B N C}(\chi, i e)$ is defined as follows: If there is an interior block $V$ of $\pi$ such that $\iota(V)=e$, then $c(\chi, \omega ; \pi, \iota)=0$. Otherwise, apply the recursive process using $\mathbb{E}$ as in Definition 2.6 to the interior blocks of $\pi$, order the remaining $\chi$-intervals by $\prec_{\chi}$ as $V_{1}, \ldots, V_{m}$, and define

$$
\Theta_{(\pi, \iota)}\left(Z_{1}, \ldots, Z_{n}\right)=\Theta_{\left.\pi\right|_{V_{1}}}\left(\left.\left(Z_{1}, \ldots, Z_{n}\right)\right|_{V_{1}}\right) \cdots \Theta_{\left.\pi\right|_{V_{m}}}\left(\left.\left(Z_{1}, \ldots, Z_{n}\right)\right|_{V_{m}}\right)
$$

where $\Theta_{\left.\pi\right|_{V_{j}}}=\mathbb{E}_{\left.\pi\right|_{V_{j}}}$ if $\iota\left(V_{j}\right)=i$ and $\Theta_{\left.\pi\right|_{V_{j}}}=\mathbb{F}_{\left.\pi\right|_{V_{j}}}$ if $\iota\left(V_{j}\right)=e$.

Notice that, as with the scalar-valued case (see [6, Remark 4.9]), $\Theta_{(\pi, \iota)}\left(Z_{1}, \ldots, Z_{n}\right)$ and $\mathbb{F}_{D}\left(Z_{1}, \ldots, Z_{n}\right)$ agree for certain $(\pi, \iota) \in \mathcal{B N C}(\chi, i e)$ and $D \in \mathcal{L R}^{\text {latcap }}(\chi, \omega)$. Indeed, given $D \in \mathcal{L R}^{\text {latcap }}(\chi, \omega)$, defining $\pi$ via the blocks of $D$ and $\iota$ via $\iota(V)=e$ if the spine of $V$ reaches the top and $\iota(V)=i$ otherwise will produce such an equality. 
If each $(\pi, \iota) \in \mathcal{B N C}(\chi, i e)$ with $\pi \leq \omega$ and $c(\chi, \omega ; \pi, \iota) \neq 0$ corresponds to some $D \in \mathcal{L R}^{\text {latcap }}(\chi, \omega)$ in the sense as described above and

$$
c(\chi, \omega ; \pi, \iota)=\sum_{\substack{D^{\prime} \in \mathcal{L} \mathcal{R}(\chi, \omega) \\ D^{\prime} \geq \operatorname{latcap} D}}(-1)^{|D|-\left|D^{\prime}\right|}
$$

for such $(\pi, \iota)$, then equations (6) and (7) coincide implying that $\left\{\left(\mathcal{A}_{k, \ell}, \mathcal{A}_{k, r}\right)\right\}_{k \in K}$ is c-bi-free over $(\mathcal{B}, \mathcal{D})$ by Theorem 5.5. Since the property that $(\pi, \iota)$ corresponds to a $D \in \mathcal{L R}^{\text {latcap }}(\chi, \omega)$ and the value of $c(\chi, \omega ; \pi, \iota)$ do not depend on the algebras $\mathcal{B}$ and $\mathcal{D}$, the result follows from the $\mathcal{B}=\mathcal{D}=\mathbb{C}$ case by [6, Lemma 4.13].

Conversely, if $\left\{\left(\mathcal{A}_{k, \ell}, \mathcal{A}_{k, r}\right)\right\}_{k \in K}$ is c-bi-free over $(\mathcal{B}, \mathcal{D})$, then equations (5) and (6) hold by Theorem 5.5. As shown in [4, Theorem 8.1.1], equation (5) is equivalent to the vanishing of mixed operator-valued bi-free cumulants. Thus we need only show that mixed operator-valued conditionally bi-free cumulants vanish. For fixed $n \geq 2, \chi:\{1, \ldots, n\} \rightarrow\{\ell, r\}, \omega:\{1, \ldots, n\} \rightarrow K$, and $Z_{k} \in \mathcal{A}_{\omega(k), \chi(k)}$, construct a $\mathcal{B}$ - $\mathcal{B}$-non-commutative probability space with a pair of $(\mathcal{B}, \mathcal{D})$-valued expectations $\left(\mathcal{A}^{\prime}, \mathbb{E}_{\mathcal{A}^{\prime}}, \mathbb{F}_{\mathcal{A}^{\prime}}, \varepsilon^{\prime}\right)$, pairs of $\mathcal{B}$-algebras $\left\{\left(\mathcal{A}_{k, \ell}^{\prime}, \mathcal{A}_{k, r}^{\prime}\right)\right\}_{k \in K}$, and elements $Z_{k}^{\prime} \in \mathcal{A}_{\omega(k), \chi(k)}^{\prime}$ such that

- for each $k \in\{1, \ldots, n\},\left\{Z_{j}^{\prime} \mid \omega(j)=\omega(k), \chi(j)=\chi(k)\right\}$ generated $\mathcal{A}_{\omega(k), \chi(k)}^{\prime}$,

- any joint operator-valued conditionally bi-free cumulant involving $Z_{1}^{\prime}, \ldots, Z_{n}^{\prime}$ containing a pair $Z_{k_{1}}^{\prime}, Z_{k_{2}}^{\prime}$ with $\omega\left(k_{1}\right) \neq \omega\left(k_{2}\right)$ is zero, and

- for each $k \in\{1, \ldots, n\}$, the joint distribution of $\left\{Z_{j}^{\prime} \mid \omega(j)=\omega(k)\right\}$ with respect to $\left(\mathbb{E}_{\mathcal{A}^{\prime}}, \mathbb{F}_{\mathcal{A}^{\prime}}\right)$ equals the joint distribution of $\left\{Z_{j} \mid \omega(j)=\omega(k)\right\}$ with respect to $(\mathbb{E}, \mathbb{F})$.

The above is possible via Lemma 6.3 by defining the operator-valued bi-free and conditionally bi-free cumulants appropriately.

By construction, $Z_{1}^{\prime}, \ldots, Z_{n}^{\prime}$ have vanishing mixed cumulants and hence satisfy equations (5) and (6) by the first part of the proof. However, since for each $k \in\{1, \ldots, n\}$, the joint distribution of $\left\{Z_{j}^{\prime} \mid \omega(j)=\omega(k)\right\}$ with respect to $\left(\mathbb{E}_{\mathcal{A}^{\prime}}, \mathbb{F}_{\mathcal{A}^{\prime}}\right)$ equals the joint distribution of $\left\{Z_{j} \mid \omega(j)=\omega(k)\right\}$ with respect to $(\mathbb{E}, \mathbb{F})$, equations (5) and (6) imply that the joint distribution of $Z_{1}, \ldots, Z_{n}$ with respect to $(\mathbb{E}, \mathbb{F})$ equals the joint distribution of $Z_{1}^{\prime}, \ldots, Z_{n}^{\prime}$ with respect to $\left(\mathbb{E}_{\mathcal{A}^{\prime}}, \mathbb{F}_{\mathcal{A}^{\prime}}\right)$. Since the operator-valued bi-free and conditionally bi-free moments completely determine the operator-valued bi-free and conditionally bi-free cumulants, and since $Z_{1}^{\prime}, \ldots, Z_{n}^{\prime}$ have vanishing mixed cumulants, the result follows.

\section{Additional properties of C-BI-Free indePEndence With amalgamation}

In this section, we collect a list of additional properties of c-bi-free independence with amalgamation and operator-valued conditionally bi-free cumulants. All of the results below are analogues of known results in the current framework with essentially the same proofs. We begin by recalling the following notation from [4, Notation 6.3.1].

Notation 7.1. Let $\chi:\{1, \ldots, n\} \rightarrow\{\ell, r\}, \pi \in \mathcal{B N C}(\chi)$, and $q \in\{1, \ldots, n\}$. We denote by $\left.\chi\right|_{\backslash q}$ the restriction of $\chi$ to the set $\{1, \ldots, n\} \backslash q$. If $q \neq n$ and $\chi(q)=\chi(q+1)$, define $\left.\pi\right|_{q=q+1} \in \mathcal{B N C}\left(\left.\chi\right|_{\backslash q}\right)$ to be the bi-non-crossing partition which results from identifying $q$ and $q+1$ in $\pi$ (i.e., if $q$ and $q+1$ are in the same block, then $\left.\pi\right|_{q=q+1}$ is obtained from $\pi$ by just removing $q$ from the block in which $q$ occurs, while if $q$ and $q+1$ are in different blocks, then $\left.\pi\right|_{q=q+1}$ is obtained from $\pi$ by merging the two blocks and then removing $q)$.

7.1. Vanishing of operator-valued cumulants. The following demonstrates that, like with many other kinds of cumulants, the operator-valued conditionally bi-free cumulants of order at least two vanish if at least one input is a $\mathcal{B}$-operator.

Proposition 7.2. Let $(\mathcal{A}, \mathbb{E}, \mathbb{F}, \varepsilon)$ be a $\mathcal{B}$ - $\mathcal{B}$-non-commutative probability space with a pair of $(\mathcal{B}, \mathcal{D})$-valued expectations, $\chi:\{1, \ldots, n\} \rightarrow\{\ell, r\}$ with $n \geq 2$, and $Z_{k} \in \mathcal{A}_{\chi(k)}$. If there exist $q \in\{1, \ldots, n\}$ and $b \in \mathcal{B}$ such that $Z_{q}=L_{b}$ if $\chi(q)=\ell$ or $Z_{q}=R_{b}$ if $\chi(q)=r$, then

$$
\kappa_{1_{\chi}}\left(Z_{1}, \ldots, Z_{n}\right)=\mathcal{K}_{1_{\chi}}\left(Z_{1}, \ldots, Z_{n}\right)=0 .
$$

Proof. The assertion that $\kappa_{1_{\chi}}\left(Z_{1}, \ldots, Z_{n}\right)=0$ was proved in [4. Proposition 6.4.1], and the other assertion will be proved by induction with the base case easily verified by direct computations. 
For the inductive step, suppose the assertion is true for all $\chi:\{1, \ldots, m\} \rightarrow\{\ell, r\}$ with $2 \leq m \leq n-1$. Fix $\chi:\{1, \ldots, n\} \rightarrow\{\ell, r\}$ and $Z_{k} \in \mathcal{A}_{\chi(k)}$. Suppose there exist $q \in\{1, \ldots, n\}$ and $b \in \mathcal{B}$ such that $\chi(q)=\ell$ and $Z_{q}=L_{b}$ (the case $\chi(q)=r$ and $Z_{q}=R_{b}$ is similar). Let

$$
p=\max \{k \in\{1, \ldots, n\} \mid \chi(k)=\ell, k<q\} .
$$

There are two cases. If $p \neq-\infty$, then by the first assertion and the induction hypothesis,

$$
\begin{aligned}
\mathcal{K}_{1_{\chi}}\left(Z_{1}, \ldots, Z_{n}\right) & =\mathcal{F}_{1_{\chi}}\left(Z_{1}, \ldots, Z_{n}\right)-\sum_{\substack{\pi \in \mathcal{B N C}(\chi) \\
\pi \neq 1_{\chi}}} \mathcal{K}_{\pi}\left(Z_{1}, \ldots, Z_{n}\right) \\
& =\mathcal{F}_{1_{\chi}}\left(Z_{1}, \ldots, Z_{n}\right)-\sum_{\substack{\pi \in \mathcal{B N C} \mathcal{C}(\chi) \\
\{q\} \in \pi}} \mathcal{K}_{\pi}\left(Z_{1}, \ldots, Z_{q-1}, L_{b}, Z_{q+1}, \ldots, Z_{n}\right) \\
& =\mathcal{F}_{1_{\chi}}\left(Z_{1}, \ldots, Z_{n}\right)-\sum_{\sigma \in \mathcal{B N \mathcal { N }}\left(\left.\chi\right|_{\backslash q}\right)} \mathcal{K}_{\sigma}\left(Z_{1}, \ldots, Z_{p} L_{b}, Z_{p+1}, \ldots, Z_{q-1}, Z_{q+1}, \ldots, Z_{n}\right)
\end{aligned}
$$

by properties of $(\kappa, \mathcal{K})$. On the other hand, we have

$$
\begin{aligned}
\mathcal{F}_{1_{\chi}}\left(Z_{1}, \ldots, Z_{n}\right) & =\mathbb{F}\left(Z_{1} \cdots Z_{q-1} L_{b} Z_{q+1} \cdots Z_{n}\right) \\
& =\mathbb{F}\left(Z_{1} \cdots Z_{p} L_{b} Z_{p+1} \cdots Z_{q-1} Z_{q+1} \cdots Z_{n}\right) \\
& =\mathcal{F}_{1_{\chi \backslash \backslash q}}\left(Z_{1}, \ldots, Z_{p} L_{b}, Z_{p+1}, \ldots, Z_{q-1}, Z_{q+1}, \ldots, Z_{n}\right) \\
& =\sum_{\sigma \in \mathcal{B} \mathcal{N C}\left(\left.\chi\right|_{\backslash q}\right)} \mathcal{K}_{\sigma}\left(Z_{1}, \ldots, Z_{p} L_{b}, Z_{p+1}, \ldots, Z_{q-1}, Z_{q+1}, \ldots, Z_{n}\right),
\end{aligned}
$$

thus the assertion is true in this case. If $p=-\infty$, then by the first assertion and the induction hypothesis,

$$
\begin{aligned}
\mathcal{K}_{1_{\chi}}\left(Z_{1}, \ldots, Z_{n}\right) & =\mathcal{F}_{1_{\chi}}\left(Z_{1}, \ldots, Z_{n}\right)-\sum_{\substack{\pi \in \mathcal{B N C}(\chi) \\
\pi \neq 1_{\chi}}} \mathcal{K}_{\pi}\left(Z_{1}, \ldots, Z_{n}\right) \\
& =\mathcal{F}_{1_{\chi}}\left(Z_{1}, \ldots, Z_{n}\right)-\sum_{\substack{\pi \in \mathcal{B N \mathcal { C }}(\chi) \\
\{q\} \in \pi}} \mathcal{K}_{\pi}\left(Z_{1}, \ldots, Z_{q-1}, L_{b}, Z_{q+1}, \ldots, Z_{n}\right) \\
& =\mathcal{F}_{1_{\chi}}\left(Z_{1}, \ldots, Z_{n}\right)-\sum_{\sigma \in \mathcal{B N \mathcal { N }}(\chi \backslash \backslash q)} b \mathcal{K}_{\sigma}\left(Z_{1}, \ldots, Z_{q-1}, Z_{q+1}, \ldots, Z_{n}\right)
\end{aligned}
$$

by properties of $(\kappa, \mathcal{K})$ as $q=\min _{\prec \chi}(\{1, \ldots, n\})$ in this case. On the other hand, we have

$$
\begin{aligned}
\mathcal{F}_{1_{\chi}}\left(Z_{1}, \ldots, Z_{n}\right) & =\mathbb{F}\left(Z_{1} \cdots Z_{q-1} L_{b} Z_{q+1} \cdots Z_{n}\right) \\
& =\mathbb{F}\left(L_{b} Z_{1} \cdots Z_{q-1} Z_{q+1} \cdots Z_{n}\right) \\
& =b \mathcal{F}_{1_{\chi \backslash \backslash q}}\left(Z_{1}, \ldots, Z_{q-1}, Z_{q+1}, \ldots, Z_{n}\right) \\
& =\sum_{\sigma \in \mathcal{B \mathcal { N C }}\left(\chi \backslash_{\backslash q}\right)} b \mathcal{K}_{\sigma}\left(Z_{1}, \ldots, Z_{q-1}, Z_{q+1}, \ldots, Z_{n}\right),
\end{aligned}
$$

thus the assertion is true in this case as well.

7.2. Operator-valued cumulants of products. Next, we analyze operator-valued conditionally bi-free cumulants involving products of operators.

Lemma 7.3. Let $(\mathcal{A}, \mathbb{E}, \mathbb{F}, \varepsilon)$ be a $\mathcal{B}$-B-non-commutative probability space with a pair of $(\mathcal{B}, \mathcal{D})$-valued expectations. If $\chi:\{1, \ldots, n\} \rightarrow\{\ell, r\}, Z_{k} \in \mathcal{A}_{\chi(k)}$, and $q \in\{1, \ldots, n-1\}$ with $\chi(q)=\chi(q+1)$, then

$$
\mathcal{K}_{\pi}\left(Z_{1}, \ldots, Z_{q-1}, Z_{q} Z_{q+1}, Z_{q+2}, \ldots, Z_{n}\right)=\sum_{\substack{\left.\sigma \in \mathcal{B N C}(\chi) \\ \sigma\right|_{q=q+1}=\pi}} \mathcal{K}_{\sigma}\left(Z_{1}, \ldots, Z_{n}\right)
$$

for all $\pi \in \mathcal{B N C}(\chi \mid \backslash q)$. 
Proof. We proceed by induction on $n$. If $n=1$, there is nothing to check. If $n=2$, then

$$
\mathcal{K}_{\left.0_{\chi}\right|_{1=2}}\left(Z_{1} Z_{2}\right)=\mathcal{K}_{\left.1_{\chi}\right|_{1=2}}\left(Z_{1} Z_{2}\right)=\mathcal{F}_{\left.1_{\chi}\right|_{1=2}}\left(Z_{1} Z_{2}\right)=\mathcal{F}_{1_{\chi}}\left(Z_{1}, Z_{2}\right)=\mathcal{K}_{0_{\chi}}\left(Z_{1}, Z_{2}\right)+\mathcal{K}_{1_{\chi}}\left(Z_{1}, Z_{2}\right)
$$

as required. Suppose the assertion holds for $n-1$, and note from [4, Theorem 6.3.5] that the analogous result also holds for the operator-valued bi-free cumulant function $\kappa$. Using the induction hypothesis and the operator-valued conditionally bi-multiplicativity of $(\kappa, \mathcal{K})$, we see for all $\pi \in \mathcal{B N C}\left(\left.\chi\right|_{\backslash q}\right) \backslash\left\{1_{\chi \mid \backslash q_{q}}\right\}$ that

$$
\mathcal{K}_{\pi}\left(Z_{1}, \ldots, Z_{q-1}, Z_{q} Z_{q+1}, Z_{q+2}, \ldots, Z_{n}\right)=\sum_{\substack{\left.\sigma \in \mathcal{B N C}(\chi) \\ \sigma\right|_{q=q+1}=\pi}} \mathcal{K}_{\sigma}\left(Z_{1}, \ldots, Z_{n}\right) .
$$

Hence,

$$
\begin{aligned}
& \mathcal{K}_{1_{\chi \backslash \backslash q}}\left(Z_{1}, \ldots, Z_{q-1}, Z_{q} Z_{q+1}, Z_{q+2}, \ldots, Z_{n}\right) \\
& =\mathcal{F}_{1_{\chi \backslash \backslash q}}\left(Z_{1}, \ldots, Z_{q-1}, Z_{q} Z_{q+1}, Z_{q+2}, \ldots, Z_{n}\right)-\sum_{\substack{\pi \in \mathcal{B N C C}(\chi \backslash \backslash q) \\
\pi \neq 1}} \mathcal{K}_{\pi \mid \backslash q}\left(Z_{1}, \ldots, Z_{q-1}, Z_{q} Z_{q+1}, Z_{q+2}, \ldots, Z_{n}\right)
\end{aligned}
$$

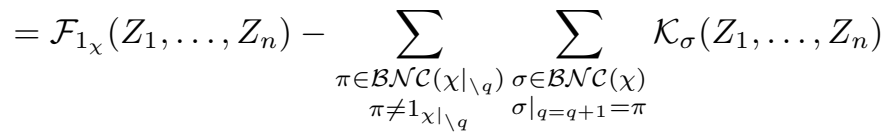

$$
\begin{aligned}
& =\sum_{\sigma \in \mathcal{B N C}(\chi)} \mathcal{K}_{\sigma}\left(Z_{1}, \ldots, Z_{n}\right)-\sum_{\substack{\sigma \in \mathcal{B N C}(\chi) \\
\sigma \mid q=q+1 \neq 1} \mid \backslash q} \mathcal{K}_{\sigma}\left(Z_{1}, \ldots, Z_{n}\right) \\
& =\sum_{\substack{\left.\sigma \in \mathcal{B N C}(\chi) \\
\sigma\right|_{q=q+1}=1_{\chi \mid} \mid q q}} \mathcal{K}_{\sigma}\left(Z_{1}, \ldots, Z_{n}\right),
\end{aligned}
$$

completing the inductive step.

Given two partitions $\pi, \sigma \in \mathcal{B N C}(\chi)$, let $\pi \vee \sigma$ denote the smallest partition in $\mathcal{B N C}(\chi)$ greater than $\pi$ and $\sigma$. Furthermore, suppose $m, n \geq 1$ with $m<n$ are fixed, and consider a sequence of integers

$$
0=k(0)<k(1)<\cdots<k(m)=n .
$$

For $\chi:\{1, \ldots, m\} \rightarrow\{\ell, r\}$, define $\widehat{\chi}:\{1, \ldots, n\} \rightarrow\{\ell, r\}$ by

$$
\widehat{\chi}(q)=\chi\left(p_{q}\right),
$$

where $p_{q}$ is the unique number in $\{1, \ldots, m\}$ such that $k\left(p_{q}-1\right)<q \leq k\left(p_{q}\right)$. Let $\widehat{0_{\chi}}$ be the partition of $\{1, \ldots, n\}$ with blocks $\{\{k(p-1)+1, \ldots, k(p)\}\}_{p=1}^{m}$. Recursively applying the previous lemma along with [4. Theorem 9.1.5] yields the following operator-valued analogue of [6, Theorem 4.22].

Theorem 7.4. Let $(\mathcal{A}, \mathbb{E}, \mathbb{F}, \varepsilon)$ be a $\mathcal{B}$-B-B-non-commutative probability space with a pair of $(\mathcal{B}, \mathcal{D})$-valued expectations. Under the above notation, we have

$$
\mathcal{K}_{1_{\chi}}\left(Z_{1} \cdots Z_{k(1)}, Z_{k(1)+1} \cdots Z_{k(2)}, \ldots, Z_{k(m-1)+1} \cdots Z_{k(m)}\right)=\sum_{\substack{\sigma \in \mathcal{B N C}(\widehat{\mathcal{\chi}}) \\ \sigma \vee \widehat{0_{\chi}}=1_{\widehat{\chi}}}} \mathcal{K}_{\sigma}\left(Z_{1}, \ldots, Z_{n}\right)
$$

for all $\chi:\{1, \ldots, m\} \rightarrow\{\ell, r\}$ and $Z_{k} \in \mathcal{A}_{\widehat{\chi}(k)}$.

7.3. Operator-valued conditionally bi-moment and bi-cumulant pairs. In [12, Subsection 3.2], the classes of operator-valued moment and cumulant functions were introduced as a tool to calculate moment expressions of elements in amalgamated free products. The c-free extension (in the special case $\mathcal{B}=\mathbb{C}$ ) was achieved in [8, Section 3] and the bi-free analogue was obtained in [4, Subsection 6.3]. In this subsection, we extend the notions of operator-valued bi-moment and bi-cumulant functions from [4, Definition 6.3.2] to pairs of functions. 
Definition 7.5. Let $(\mathcal{A}, \mathbb{E}, \mathbb{F}, \varepsilon)$ be a $\mathcal{B}$ - $\mathcal{B}$-non-commutative probability space with a pair of $(\mathcal{B}, \mathcal{D})$-valued expectations, and let

$$
\phi: \bigcup_{n \geq 1} \bigcup_{\chi:\{1, \ldots, n\} \rightarrow\{\ell, r\}} \mathcal{B N C}(\chi) \times \mathcal{A}_{\chi(1)} \times \cdots \times \mathcal{A}_{\chi(n)} \rightarrow \mathcal{B}
$$

and

$$
\Phi: \bigcup_{n \geq 1} \bigcup_{\chi:\{1, \ldots, n\} \rightarrow\{\ell, r\}} \mathcal{B N C}(\chi) \times \mathcal{A}_{\chi(1)} \times \cdots \times \mathcal{A}_{\chi(n)} \rightarrow \mathcal{D}
$$

be an operator-valued conditionally bi-multiplicative pair.

(1) We say that $(\phi, \Phi)$ is an operator-valued conditionally bi-moment pair if whenever $\chi:\{1, \ldots, n\} \rightarrow$ $\{\ell, r\}$ is such that there exists a $q \in\{1, \ldots, n-1\}$ with $\chi(q)=\chi(q+1)$, then

$$
\phi_{1_{\chi \backslash \backslash q}}\left(Z_{1}, \ldots, Z_{q-1}, Z_{q} Z_{q+1}, Z_{q+2}, \ldots, Z_{n}\right)=\phi_{1_{\chi}}\left(Z_{1}, \ldots, Z_{n}\right)
$$

and

$$
\Phi_{1_{\chi \backslash \backslash q}}\left(Z_{1}, \ldots, Z_{q-1}, Z_{q} Z_{q+1}, Z_{q+2}, \ldots, Z_{n}\right)=\Phi_{1_{\chi}}\left(Z_{1}, \ldots, Z_{n}\right)
$$

for all $Z_{k} \in \mathcal{A}_{\chi(k)}$.

(2) We say that $(\phi, \Phi)$ is an operator-valued conditionally bi-cumulant pair if whenever $\chi:\{1, \ldots, n\} \rightarrow$ $\{\ell, r\}$ is such that there exists a $q \in\{1, \ldots, n-1\}$ with $\chi(q)=\chi(q+1)$, then

$$
\phi_{1_{\chi \mid \backslash q}}\left(Z_{1}, \ldots, Z_{q-1}, Z_{q} Z_{q+1}, Z_{q+2}, \ldots, Z_{n}\right)=\phi_{1_{\chi}}\left(Z_{1}, \ldots, Z_{n}\right)+\sum_{\substack{\pi \in \mathcal{B N C \mathcal { C }}(\chi) \\|\pi|=2, q \chi_{\pi} q+1}} \phi_{\pi}\left(Z_{1}, \ldots, Z_{n}\right)
$$

and

$$
\Phi_{1_{\chi \backslash \backslash q}}\left(Z_{1}, \ldots, Z_{q-1}, Z_{q} Z_{q+1}, Z_{q+2}, \ldots, Z_{n}\right)=\Phi_{1_{\chi}}\left(Z_{1}, \ldots, Z_{n}\right)+\sum_{\substack{\pi \in \mathcal{B N \mathcal { N }}(\chi) \\|\pi|=2, q \chi_{\pi} q+1}} \Phi_{\pi}\left(Z_{1}, \ldots, Z_{n}\right)
$$

for all $Z_{k} \in \mathcal{A}_{\chi(k)}$, where $\Phi_{\pi}\left(Z_{1}, \ldots, Z_{n}\right)$ is defined by the operator-valued conditionally bi-multiplicativity of $(\phi, \Phi)$ using $\phi$ for an interior block and $\Phi$ for an exterior block.

The following demonstrates that the two notions of pairs of functions are naturally related by summing over bi-non-crossing partitions.

Theorem 7.6. Let $(\mathcal{A}, \mathbb{E}, \mathbb{F}, \varepsilon)$ be a $\mathcal{B}$-B-B-non-commutative probability space with a pair of $(\mathcal{B}, \mathcal{D})$-valued expectations. If

$$
\phi, \psi: \bigcup_{n \geq 1} \bigcup_{\chi:\{1, \ldots, n\} \rightarrow\{\ell, r\}} \mathcal{B N C}(\chi) \times \mathcal{A}_{\chi(1)} \times \cdots \times \mathcal{A}_{\chi(n)} \rightarrow \mathcal{B}
$$

and

$$
\Phi, \Psi: \bigcup_{n \geq 1} \bigcup_{\chi:\{1, \ldots, n\} \rightarrow\{\ell, r\}} \mathcal{B N C}(\chi) \times \mathcal{A}_{\chi(1)} \times \cdots \times \mathcal{A}_{\chi(n)} \rightarrow \mathcal{D}
$$

are such that $(\phi, \Phi)$ and $(\psi, \Psi)$ are operator-valued conditionally bi-multiplicative related by the formulae

$$
\phi_{\pi}\left(Z_{1}, \ldots, Z_{n}\right)=\sum_{\substack{\sigma \in \mathcal{B} \mathcal{N C}(\chi) \\ \sigma \leq \pi}} \psi_{\sigma}\left(Z_{1}, \ldots, Z_{n}\right)
$$

and

$$
\Phi_{\pi}\left(Z_{1}, \ldots, Z_{n}\right)=\sum_{\substack{\sigma \in \mathcal{B N C}(\chi) \\ \sigma \leq \pi}} \Psi_{\sigma}\left(Z_{1}, \ldots, Z_{n}\right)
$$

for all $\chi:\{1, \ldots, n\} \rightarrow\{\ell, r\}, \pi \in \mathcal{B N C}(\chi)$, and $Z_{k} \in \mathcal{A}_{\chi(k)}$, then $(\phi, \Phi)$ is an operator-valued conditionally bi-moment pair if and only if $(\psi, \Psi)$ is an operator-valued conditionally bi-cumulant pair. 
Proof. Let $\chi:\{1, \ldots, n\} \rightarrow\{\ell, r\}$ be such that there exists a $q \in\{1, \ldots, n-1\}$ with $\chi(q)=\chi(q+1)$. If $(\psi, \Psi)$ is an operator-valued conditionally bi-cumulant pair, then

$$
\phi_{1_{\chi \backslash \backslash q}}\left(Z_{1}, \ldots, Z_{q-1}, Z_{q} Z_{q+1}, Z_{q+2}, \ldots, Z_{n}\right)=\phi_{1_{\chi}}\left(Z_{1}, \ldots, Z_{n}\right)
$$

for all $Z_{k} \in \mathcal{A}_{\chi(k)}$ by [4, Theorem 6.3.5]. On the other hand, using the operator-valued conditionally bi-multiplicativity of $(\psi, \Psi)$ and part (2) of Definition 7.5. we have

$$
\Psi_{\pi}\left(Z_{1}, \ldots, Z_{q-1}, Z_{q} Z_{q+1}, \ldots, Z_{n}\right)=\sum_{\substack{\left.\sigma \in \mathcal{B N C}(\chi) \\ \sigma\right|_{q=q+1}=\pi}} \Psi_{\sigma}\left(Z_{1}, \ldots, Z_{n}\right)
$$

for all $\pi \in \mathcal{B N C}\left(\left.\chi\right|_{\backslash q}\right)$, and it follows from the same calculations as in the first part of the proof of 4 , Theorem 6.3.5] that

$$
\Phi_{1_{\chi \backslash \backslash q}}\left(Z_{1}, \ldots, Z_{q-1}, Z_{q} Z_{q+1}, Z_{q+2}, \ldots, Z_{n}\right)=\Phi_{1_{\chi}}\left(Z_{1}, \ldots, Z_{n}\right)
$$

for all $Z_{k} \in \mathcal{A}_{\chi(k)}$.

Conversely, if $(\phi, \Phi)$ is an operator-valued conditionally bi-moment pair, then

$$
\psi_{1_{\chi \backslash \backslash q}}\left(Z_{1}, \ldots, Z_{q-1}, Z_{q} Z_{q+1}, Z_{q+2}, \ldots, Z_{n}\right)=\psi_{1_{\chi}}\left(Z_{1}, \ldots, Z_{n}\right)+\sum_{\substack{\pi \in \mathcal{B N \mathcal { N }}(\chi) \\|\pi|=2, q \chi_{\pi} q+1}} \psi_{\pi}\left(Z_{1}, \ldots, Z_{n}\right)
$$

for all $Z_{k} \in \mathcal{A}_{\chi(k)}$ by [4, Theorem 6.3.5], and it follows from the same induction arguments as in the second part of the proof of [4, Theorem 6.3.5] that

$$
\Psi_{1_{\chi \backslash \backslash} q}\left(Z_{1}, \ldots, Z_{q-1}, Z_{q} Z_{q+1}, Z_{q+2}, \ldots, Z_{n}\right)=\Psi_{1_{\chi}}\left(Z_{1}, \ldots, Z_{n}\right)+\sum_{\substack{\pi \in \mathcal{B N \mathcal { C }}(\chi) \\|\pi|=2, q \chi_{\pi} q+1}} \Psi_{\pi}\left(Z_{1}, \ldots, Z_{n}\right)
$$

for all $Z_{k} \in \mathcal{A}_{\chi(k)}$.

As an immediate corollary, we have the following expected result.

Corollary 7.7. Let $(\mathcal{A}, \mathbb{E}, \mathbb{F}, \varepsilon)$ be a $\mathcal{B}-\mathcal{B}$-non-commutative probability space with a pair of $(\mathcal{B}, \mathcal{D})$-valued expectations. The operator-valued conditionally bi-free moment pair $(\mathcal{E}, \mathcal{F})$ is an operator-valued conditionally bi-moment pair and the operator-valued conditionally bi-free cumulant pair $(\kappa, \mathcal{K})$ is an operator-valued conditionally bi-cumulant pair.

7.4. Operations on operator-valued cumulants. The following two results demonstrate how certain operations affect operator-valued conditionally bi-free cumulants under certain conditions. The same effects in the scalar-valued setting were observed in [6, Lemmata 4.17 and 4.18].

Lemma 7.8. Let $(\mathcal{A}, \mathbb{E}, \mathbb{F}, \varepsilon)$ be a $\mathcal{B}$ - $\mathcal{B}$-non-commutative probability space with a pair of $(\mathcal{B}, \mathcal{D})$-valued expectations. Let $\chi:\{1, \ldots, n\} \rightarrow\{\ell, r\}$ be such that $\chi\left(k_{0}\right)=\ell$ and $\chi\left(k_{0}+1\right)=r$ for some $k_{0} \in\{1, \ldots, n-1\}$, and let $X \in \mathcal{A}_{\ell}$ and $Y \in \mathcal{A}_{r}$ be such that $\mathbb{E}\left(Z X Y Z^{\prime}\right)=\mathbb{E}\left(Z Y X Z^{\prime}\right)$ and $\mathbb{F}\left(Z X Y Z^{\prime}\right)=\mathbb{F}\left(Z Y X Z^{\prime}\right)$ for all $Z, Z^{\prime} \in \mathcal{A}$. Define $\chi^{\prime}:\{1, \ldots, n\} \rightarrow\{\ell, r\}$ by

$$
\chi^{\prime}(k)= \begin{cases}r & \text { if } k=k_{0} \\ \ell & \text { if } k=k_{0}+1 \\ \chi(k) & \text { otherwise }\end{cases}
$$

Then

$$
\mathcal{K}_{1_{\chi}}\left(Z_{1}, \ldots, Z_{k_{0}-1}, X, Y, Z_{k_{0}+2}, \ldots, Z_{n}\right)=\mathcal{K}_{1_{\chi^{\prime}}}\left(Z_{1}, \ldots, Z_{k_{0}-1}, Y, X, Z_{k_{0}+2}, \ldots, Z_{n}\right)
$$

for all $Z_{1}, \ldots, Z_{k_{0}-1}, Z_{k_{0}+2}, \ldots, Z_{n} \in \mathcal{A}$ with $Z_{k} \in \mathcal{A}_{\chi(k)}$.

Proof. By repeatedly applying Definition 4.7 and using Definition 2.7 for interior blocks, we have

$$
\mathcal{K}_{1_{\chi}}\left(Z_{1}, \ldots, Z_{k_{0}-1}, X, Y, Z_{k_{0}+2}, \ldots, Z_{n}\right)=\sum_{(\pi, \iota) \in \mathcal{B N C}(\chi, i e)} d(\chi ; \pi, \iota) \Theta_{(\pi, \iota)}\left(Z_{1}, \ldots, Z_{k_{0}-1}, X, Y, Z_{k_{0}+2}, \ldots, Z_{n}\right)
$$


for some integer coefficients such that $d(\chi ; \pi, \iota)=0$ if there is an interior block $V$ of $\pi$ with $i(V)=e$, and $\Theta_{(\pi, \iota)}\left(Z_{1}, \ldots, Z_{k_{0}-1}, X, Y, Z_{k_{0}+2}, \ldots, Z_{n}\right)$ for non-zero $d(\chi ; \pi, \iota)$ is defined as in the proof of Theorem 6.4 . Similarly, we have

$$
\begin{aligned}
\mathcal{K}_{1_{\chi^{\prime}}}\left(Z_{1}, \ldots, Z_{k_{0}-1}, Y, X, Z_{k_{0}+2}, \ldots, Z_{n}\right) & \\
= & \sum_{\left(\pi^{\prime}, \iota^{\prime}\right) \in \mathcal{B N C}\left(\chi^{\prime}, i e\right)} d\left(\chi^{\prime} ; \pi^{\prime}, \iota^{\prime}\right) \Theta_{\left(\pi^{\prime}, \iota^{\prime}\right)}\left(Z_{1}, \ldots, Z_{k_{0}-1}, Y, X, Z_{k_{0}+2}, \ldots, Z_{n}\right) .
\end{aligned}
$$

Note that there is a bijection from $\mathcal{B N C}(\chi, i e)$ to $\mathcal{B N C}\left(\chi^{\prime}, i e\right)$ which sends a pair $(\pi, \iota)$ to the pair $\left(\pi^{\prime}, \iota^{\prime}\right)$ obtained by swapping $k_{0}$ and $k_{0}+1$. Furthermore, as only the lattice structure affects the expansions of the above formulae (alternatively, by appealing to the scalar-valued case in [6, Subsection 4.2$]), d(\chi ; \pi, \iota)=$ $d\left(\chi^{\prime} ; \pi^{\prime}, \iota^{\prime}\right)$ under this bijection.

To complete the proof, it suffices to show that

$$
\Theta_{(\pi, \iota)}\left(Z_{1}, \ldots, Z_{k_{0}-1}, X, Y, Z_{k_{0}+2}, \ldots, Z_{n}\right)=\Theta_{\left(\pi^{\prime}, \iota^{\prime}\right)}\left(Z_{1}, \ldots, Z_{k_{0}-1}, Y, X, Z_{k_{0}+2}, \ldots, Z_{n}\right)
$$

for all $(\pi, \iota) \in \mathcal{B N C}(\chi, i e)$. If $k_{0}$ and $k_{0}+1$ are in the same block of $\pi$, then one may reduce

$$
\Theta_{(\pi, \iota)}\left(Z_{1}, \ldots, Z_{k_{0}-1}, X, Y, Z_{k_{0}+2}, \ldots, Z_{n}\right)
$$

to an expression involving $\mathbb{E}\left(Z X Y Z^{\prime}\right)$ or $\mathbb{F}\left(Z X Y Z^{\prime}\right)$ for some $Z, Z^{\prime} \in \mathcal{A}$, commute $X$ and $Y$ to get $\mathbb{E}\left(Z Y X Z^{\prime}\right)$ or $\mathbb{F}\left(Z Y X Z^{\prime}\right)$, and undo the reduction to obtain

$$
\Theta_{\left(\pi^{\prime}, \iota^{\prime}\right)}\left(Z_{1}, \ldots, Z_{k_{0}-1}, Y, X, Z_{k_{0}+2}, \ldots, Z_{n}\right) .
$$

On the other hand, if $k_{0}$ and $k_{0}+1$ are in different blocks of $\pi$, then the reductions of

$$
\Theta_{(\pi, \iota)}\left(Z_{1}, \ldots, Z_{k_{0}-1}, X, Y, Z_{k_{0}+2}, \ldots, Z_{n}\right) \quad \text { and } \quad \Theta_{\left(\pi^{\prime}, \iota^{\prime}\right)}\left(Z_{1}, \ldots, Z_{k_{0}-1}, Y, X, Z_{k_{0}+2}, \ldots, Z_{n}\right)
$$

agree. Consequently, the proof is complete.

Lemma 7.9. Let $(\mathcal{A}, \mathbb{E}, \mathbb{F}, \varepsilon)$ be a $\mathcal{B}-\mathcal{B}$-non-commutative probability space with a pair of $(\mathcal{B}, \mathcal{D})$-valued expectations. Let $\chi:\{1, \ldots, n\} \rightarrow\{\ell, r\}$ be such that $\chi(n)=\ell$, and let $X \in \mathcal{A}_{\ell}$ and $Y \in \mathcal{A}_{r}$ be such that $\mathbb{E}(Z X)=\mathbb{E}(Z Y)$ and $\mathbb{F}(Z X)=\mathbb{F}(Z Y)$ for all $Z \in \mathcal{A}$. Define $\chi^{\prime}:\{1, \ldots, n\} \rightarrow\{\ell, r\}$ by

$$
\chi^{\prime}(k)=\left\{\begin{array}{ll}
r & \text { if } k=n \\
\chi(k) & \text { otherwise }
\end{array} .\right.
$$

Then

$$
\mathcal{K}_{1_{\chi}}\left(Z_{1}, \ldots, Z_{n-1}, X\right)=\mathcal{K}_{1_{\chi^{\prime}}}\left(Z_{1}, \ldots, Z_{n-1}, Y\right)
$$

for all $Z_{1}, \ldots, Z_{n-1} \in \mathcal{A}$ with $Z_{k} \in \mathcal{A}_{\chi(k)}$.

Proof. By the same arguments as the previous lemma, we have

$$
d(\chi ; \pi, \iota) \Theta_{(\pi, \iota)}\left(Z_{1}, \ldots, Z_{n-1}, X\right)=d\left(\chi^{\prime} ; \pi^{\prime}, \iota^{\prime}\right) \Theta_{\left(\pi^{\prime}, \iota^{\prime}\right)}\left(Z_{1}, \ldots, Z_{n-1}, Y\right)
$$

for all $(\pi, \iota) \in \mathcal{B N C}(\chi, i e)$, where $\left(\pi^{\prime}, \iota^{\prime}\right)$ is obtained from $(\pi, \iota)$ by changing the last node from a left node to a right node. Consequently, the proof is complete.

In [4, Theorem 10.2.1], it was demonstrated that for a family of $\mathcal{B}$-algebras with certain conditions, bi-free independence over $\mathcal{B}$ can be deduced from free independence over $\mathcal{B}$ of either the left $\mathcal{B}$-algebras or the right $\mathcal{B}$-algebras. The conditionally bi-free analogue in the scalar-valued setting was proved in [6, Theorem 4.20].

Theorem 7.10. Let $(\mathcal{A}, \mathbb{E}, \mathbb{F}, \varepsilon)$ be a $\mathcal{B}$-B-non-commutative probability space with a pair of $(\mathcal{B}, \mathcal{D})$-valued expectations. If $\left\{\left(\mathcal{A}_{k, \ell}, \mathcal{A}_{k, r}\right)\right\}_{k \in K}$ is a family of pairs of $\mathcal{B}$-algebras in $(\mathcal{A}, \mathbb{E}, \mathbb{F}, \varepsilon)$ such that

(1) $\mathcal{A}_{m, \ell}$ and $\mathcal{A}_{n, r}$ commute for all $m, n \in K$,

(2) for every $Y \in \mathcal{A}_{k, r}$, there exists an $X \in \mathcal{A}_{k, \ell}$ such that $\mathbb{E}(Z Y)=\mathbb{E}(Z X)$ and $\mathbb{F}(Z Y)=\mathbb{F}(Z X)$ for all $Z \in \mathcal{A}$,

then $\left\{\left(\mathcal{A}_{k, \ell}, \mathcal{A}_{k, r}\right)\right\}_{k \in K}$ is c-bi-free over $(\mathcal{B}, \mathcal{D})$ if and only if $\left\{\mathcal{A}_{k, \ell}\right\}_{k \in K}$ is c-free over $(\mathcal{B}, \mathcal{D})$. Consequently, if $\left\{\mathcal{A}_{k, \ell}\right\}_{k \in K}$ is c-free over $(\mathcal{B}, \mathcal{D})$, then $\left\{\mathcal{A}_{k, r}\right\}_{k \in K}$ is c-free over $(\mathcal{B}, \mathcal{D})$. 
Proof. If $\left\{\left(\mathcal{A}_{k, \ell}, \mathcal{A}_{k, r}\right)\right\}_{k \in K}$ is c-bi-free over $(\mathcal{B}, \mathcal{D})$, then it is clear that $\left\{\mathcal{A}_{k, \ell}\right\}_{k \in K}$ is c-free over $(\mathcal{B}, \mathcal{D})$ and $\left\{\mathcal{A}_{k, r}\right\}_{k \in K}$ is c-free over $(\mathcal{B}, \mathcal{D})$.

Suppose $\left\{\mathcal{A}_{k, \ell}\right\}_{k \in K}$ is c-free over $(\mathcal{B}, \mathcal{D})$. Given a mixed operator-valued bi-free or conditionally bifree cumulant from $\left\{\left(\mathcal{A}_{k, \ell}, \mathcal{A}_{k, r}\right)\right\}_{k \in K}$, assumptions (1) and (2) imply that we may apply the previous two lemmata (or [11, Lemmata 2.16 and 2.17]) and reduce it to a mixed operator-valued free or conditionally free cumulant from $\left\{\mathcal{A}_{k, \ell}\right\}_{k \in K}$, which vanishes by c-free independence over $(\mathcal{B}, \mathcal{D})$. Thus the result follows from Theorem 6.4.

\section{The operator-VALUEd CONDITIONALly BI-FREE PARTial $\mathcal{R}$-TRANSFORM}

In this section, we construct an operator-valued conditionally bi-free partial $\mathcal{R}$-transform generalizing 6. Definition 5.3] and relate it to certain operator-valued moment transforms. As we will see in the proof, such transform is a function of three $\mathcal{B}$-variables instead of two by a similar reason as the operator-valued bi-free partial $\mathcal{R}$-transform developed in [11, Section 5]. As in [11, Section 5], our proof will follow the combinatorial techniques used in [10, Section 7]. In that which follows, all algebras are assumed to be Banach algebras.

Definition 8.1. A Banach $\mathcal{B}-\mathcal{B}$-non-commutative probability space with a pair of $(\mathcal{B}, \mathcal{D})$-valued expectations is a $\mathcal{B}$ - $\mathcal{B}$-non-commutative probability space with a pair of $(\mathcal{B}, \mathcal{D})$-valued expectations $(\mathcal{A}, \mathbb{E}, \mathbb{F}, \varepsilon)$ such that $\mathcal{A}, \mathcal{B}$, and $\mathcal{D}$ are Banach algebras, and $\left.\varepsilon\right|_{\mathcal{B} \otimes 1}, \varepsilon_{1 \otimes \mathcal{B}^{\text {op }}}, \mathbb{E}$, and $\mathbb{F}$ are bounded.

Let $(\mathcal{A}, \mathbb{E}, \mathbb{F}, \varepsilon)$ be a Banach $\mathcal{B}$ - $\mathcal{B}$-non-commutative probability space with a pair of $(\mathcal{B}, \mathcal{D})$-valued expectations, let $Z_{\ell} \in \mathcal{A}_{\ell}, Z_{r} \in \mathcal{A}_{r}$, and let $b, d \in \mathcal{B}$. Consider the following series:

$$
\begin{aligned}
& M_{Z_{\ell}}^{\ell}(b)=1+\sum_{m \geq 1} \mathbb{E}\left(\left(L_{b} Z_{\ell}\right)^{m}\right), \\
& \mathbb{M}_{Z_{\ell}}^{\ell}(b)=1+\sum_{m \geq 1} \mathbb{F}\left(\left(L_{b} Z_{\ell}\right)^{m}\right), \\
& \mathcal{C}_{Z_{\ell}}^{\ell}(b)=1+\sum_{m \geq 1} \mathcal{K}_{1_{\chi_{m}, 0}}(\underbrace{L_{b} Z_{\ell}, \ldots, L_{b} Z_{\ell}}_{m \text { entries }}),
\end{aligned}
$$

and

$$
\begin{aligned}
M_{Z_{r}}^{r}(d) & =1+\sum_{n \geq 1} \mathbb{E}\left(\left(R_{d} Z_{r}\right)^{n}\right), \\
\mathbb{M}_{Z_{r}}^{r}(d) & =1+\sum_{n \geq 1} \mathbb{F}\left(\left(R_{d} Z_{r}\right)^{n}\right), \\
\mathcal{C}_{Z_{r}}^{r}(d) & =1+\sum_{n \geq 1} \mathcal{K}_{1_{\chi_{0, n}}}(\underbrace{R_{d} Z_{r}, \ldots, R_{d} Z_{r}}_{n \text { entries }}) .
\end{aligned}
$$

By similar arguments as in [11, Remark 5.2], all of the series above converge absolutely for $b, d$ sufficiently small.

In the proof of Theorem 8.3 below, the following relations will be used. Since the statements are slightly different than the ones in the literature (see, e.g., [1, equation (15)]), we will provide a proof.

Lemma 8.2. Under the above assumptions and notation, we have

$$
\mathcal{C}_{Z_{\ell}}^{\ell}\left(M_{Z_{\ell}}^{\ell}(b) b\right)=1+M_{Z_{\ell}}^{\ell}(b)-M_{Z_{\ell}}^{\ell}(b) \mathbb{M}_{Z_{\ell}}^{\ell}(b)^{-1} \quad \text { and } \quad \mathcal{C}_{Z_{r}}^{r}\left(d M_{Z_{r}}^{r}(d)\right)=1+M_{Z_{r}}^{r}(d)-\mathbb{M}_{Z_{r}}^{r}(d)^{-1} M_{Z_{r}}^{r}(d)
$$

for $b, d$ sufficiently small.

Proof. For $m \geq 1$, we have

$$
\mathbb{F}\left(\left(L_{b} Z_{\ell}\right)^{m}\right)=\sum_{\pi \in \mathcal{B} \mathcal{N C}\left(\chi_{m, 0}\right)} \mathcal{K}_{\pi}(\underbrace{L_{b} Z_{\ell}, \ldots, L_{b} Z_{\ell}}_{m \text { entries }}) .
$$

For every partition $\pi \in \mathcal{B N C}\left(\chi_{m, 0}\right)$, let $W_{\pi}$ denote the block of $\pi$ containing 1 , which is necessarily an exterior block. Rearrange the above sum (which may be done as it converges absolutely) by first choosing 
$s \in\{1, \ldots, m\}, W=\left\{1=w_{1}<\cdots<w_{s}\right\} \subset\{1, \ldots, m\}$, and then summing over all $\pi \in \mathcal{B N C}\left(\chi_{m, 0}\right)$ such that $W_{\pi}=W$, i.e.,

$$
\mathbb{F}\left(\left(L_{b} Z_{\ell}\right)^{m}\right)=\sum_{s=1}^{m} \sum_{\begin{array}{c}
W=\left\{1=w_{1}<\cdots<w_{s}\right\} \\
W \subset\{1, \ldots, m\}
\end{array}} \sum_{\substack{\pi \in \mathcal{B N C}\left(\chi_{m, 0}\right) \\
W_{\pi}=W}} \mathcal{K}_{\pi}(\underbrace{L_{b} Z_{\ell}, \ldots, L_{b} Z_{\ell}}_{m \text { entries }}) .
$$

Furthermore, using operator-valued conditionally bi-multiplicative properties, the right-most sum in the above expression is

$$
b \mathcal{K}_{1_{\chi_{s}, 0}}\left(Z_{\ell}, L_{b_{2}} Z_{\ell}, \ldots, L_{b_{s}} Z_{\ell}\right) \mathbb{F}\left(\left(L_{b} Z_{\ell}\right)^{m-w_{s}}\right),
$$

where $b_{k}=\mathbb{E}\left(\left(L_{b} Z_{\ell}\right)^{w_{k}-w_{k-1}-1}\right) b$. Thus

$$
\mathbb{F}\left(\left(L_{b} Z_{\ell}\right)^{m}\right)=\sum_{\substack{1 \leq s \leq m \\ 0 \leq i_{1}, \ldots, i_{s} \leq m \\ i_{1}+\cdots+i_{s}=m-s}} b \mathcal{K}_{1_{\chi_{s, 0}}}\left(Z_{\ell}, L_{f\left(i_{1}\right)} Z_{\ell}, \ldots, L_{f\left(i_{s-1}\right)} Z_{\ell}\right) \mathbb{F}\left(\left(L_{b} Z_{\ell}\right)^{i_{s}}\right),
$$

where $f(k)=\mathbb{E}\left(\left(L_{b} Z_{\ell}\right)^{k}\right) b$. Note that

$$
\sum_{k \geq 0} f(k)=M_{Z_{\ell}}^{\ell}(b) b \quad \text { and } \quad \sum_{k \geq 0} \mathbb{F}\left(\left(L_{b} Z_{\ell}\right)^{k}\right)=\mathbb{M}_{Z_{\ell}}^{\ell}(b) .
$$

Consequently, we obtain

$$
\sum_{m \geq 1} \mathbb{F}\left(\left(L_{b} Z_{\ell}\right)^{m}\right)=\sum_{s \geq 1} b \mathcal{K}_{1_{\chi_{s}, 0}}\left(Z_{\ell}, L_{M_{Z_{\ell}}^{\ell}(b) b} Z_{\ell}, \ldots, L_{M_{Z_{\ell}}^{\ell}(b) b} Z_{\ell}\right) \mathbb{M}_{Z_{\ell}}^{\ell}(b),
$$

therefore

$$
M_{Z_{\ell}}^{\ell}(b) \mathbb{M}_{Z_{\ell}}^{\ell}(b)=M_{Z_{\ell}}^{\ell}(b)+\sum_{s \geq 1} \mathcal{K}_{1_{\chi_{s}, 0}}\left(L_{M_{Z_{\ell}}^{\ell}(b) b} Z_{\ell}, L_{M_{Z_{\ell}}^{\ell}(b) b} Z_{\ell}, \ldots, L_{M_{Z_{\ell}}^{\ell}(b) b} Z_{\ell}\right) \mathbb{M}_{Z_{\ell}}^{\ell}(b),
$$

and hence

$$
M_{Z_{\ell}}^{\ell}(b)-M_{Z_{\ell}}^{\ell}(b) \mathbb{M}_{Z_{\ell}}^{\ell}(b)^{-1}=\mathcal{C}_{Z_{\ell}}^{\ell}\left(M_{Z_{\ell}}^{\ell}(b) b\right)-1,
$$

which proves the first equation. The proof for the second equation is nearly identical once one uses the fact that $d \mapsto R_{d}$ is an anti-homomorphism.

For $b, c, d \in \mathcal{B}, Z_{\ell} \in \mathcal{A}_{\ell}$, and $Z_{r} \in \mathcal{A}_{r}$, consider the following series of the pair $\left(Z_{\ell}, Z_{r}\right)$ :

$$
\begin{aligned}
M_{\left(Z_{\ell}, Z_{r}\right)}(b, c, d)= & \sum_{m, n \geq 0} \mathbb{E}\left(\left(L_{b} Z_{\ell}\right)^{m}\left(R_{d} Z_{r}\right)^{n} R_{c}\right), \\
\mathbb{M}_{\left(Z_{\ell}, Z_{r}\right)}(b, c, d)= & \sum_{m, n \geq 0} \mathbb{F}\left(\left(L_{b} Z_{\ell}\right)^{m}\left(R_{d} Z_{r}\right)^{n} R_{c}\right), \\
\mathcal{C}_{\left(Z_{\ell}, Z_{r}\right)}(b, c, d)= & c+\sum_{m \geq 1} \mathcal{K}_{1_{\chi_{m}, 0}}(\underbrace{L_{b} Z_{\ell}, \ldots, L_{b} Z_{\ell}}_{m-1 \text { entries }}, L_{b} Z_{\ell} L_{c}) \\
& +\sum_{\substack{m \geq 0 \\
n \geq 1}} \mathcal{K}_{1_{\chi_{m}, n}}(\underbrace{L_{b} Z_{\ell}, \ldots, L_{b} Z_{\ell}}_{m \text { entries }}, \underbrace{R_{d} Z_{r}, \ldots, R_{d} Z_{r}}_{n-1 \text { entries }}, R_{d} Z_{r} R_{c}),
\end{aligned}
$$

which converge absolutely for $b, c, d$ sufficiently small by similar arguments as in [11, Remarks 5.2 and 5.5].

Notice if $\left(Z_{1, \ell}, Z_{1, r}\right)$ and $\left(Z_{2, \ell}, Z_{2, r}\right)$ are c-bi-free over $(\mathcal{B}, \mathcal{D})$, then

$$
\mathcal{C}_{\left(Z_{1, \ell}+Z_{2, \ell}, Z_{1, r}+Z_{2, r}\right)}(b, c, d)-c=\left(\mathcal{C}_{\left(Z_{1, \ell}, Z_{1, r}\right)}(b, c, d)-c\right)+\left(\mathcal{C}_{\left(Z_{2, \ell}, Z_{2, r}\right)}(b, c, d)-c\right)
$$

by Theorem 6.4 that is, $\mathcal{C}_{\left(Z_{\ell}, Z_{r}\right)}(b, c, d)-c$ is an operator-valued conditionally bi-free partial $\mathcal{R}$-transform.

Theorem 8.3. Let $(\mathcal{A}, \mathbb{E}, \mathbb{F}, \varepsilon)$ be a Banach $\mathcal{B}-\mathcal{B}$-non-commutative probability space with a pair of $(\mathcal{B}, \mathcal{D})$ valued expectations, let $Z_{\ell} \in \mathcal{A}_{\ell}$, and let $Z_{r} \in \mathcal{A}_{r}$. Then

$$
\begin{aligned}
& \mathcal{C}_{\left(Z_{\ell}, Z_{r}\right)}\left(M_{Z_{\ell}}^{\ell}(b) b, M_{\left(Z_{\ell}, Z_{r}\right)}(b, c, d), d M_{Z_{r}}^{r}(d)\right) \\
& =M_{Z_{\ell}}^{\ell}(b) \mathbb{M}_{Z_{\ell}}^{\ell}(b)^{-1} \mathbb{M}_{\left(Z_{\ell}, Z_{r}\right)}(b, c, d) \mathbb{M}_{Z_{r}}^{r}(d)^{-1} M_{Z_{r}}^{r}(d)+M_{\left(Z_{\ell}, Z_{r}\right)}(b, c, d) \\
& \quad+M_{Z_{\ell}}^{\ell}(b)\left(1-\mathbb{M}_{Z_{\ell}}^{\ell}(b)^{-1}\right) M_{\left(Z_{\ell}, Z_{r}\right)}(b, c, d)+M_{\left(Z_{\ell}, Z_{r}\right)}(b, c, d)\left(1-\mathbb{M}_{Z_{r}}^{r}(d)^{-1}\right) M_{Z_{r}}^{r}(d)-M_{Z_{\ell}}^{\ell}(b) c M_{Z_{r}}^{r}(d)
\end{aligned}
$$

for $b, c, d \in \mathcal{B}$ sufficiently small. 
Remark 8.4. Note that if $\mathcal{B}=\mathcal{D}=\mathbb{C}, b=z, d=w$, and $c=1$, then Theorem 8.3 produces exactly equation (9) in [6. Theorem 5.6] for the scalar-valued setting. On the other hand, if $\mathcal{B}=\mathcal{D}$ and $\mathbb{E}=\mathbb{F}$, then Theorem 8.3 produces exactly equation (10) in [11, Theorem 5.6] for the operator-valued bi-free setting.

Proof of Theorem 8.3. For $m, n \geq 1$, let $\mathcal{B N C}_{\mathrm{vs}}\left(\chi_{m, n}\right)$ denote the set of bi-non-crossing partitions where no block contains both left and right nodes. Using operator-valued conditionally bi-multiplicativity, we obtain

$$
\begin{aligned}
& \mathbb{F}\left(\left(L_{b} Z_{\ell}\right)^{m}\left(R_{d} Z_{r}\right)^{n} R_{c}\right) \\
& =\sum_{\pi \in \mathcal{B} \mathcal{N} \mathcal{C}_{\text {vs }}\left(\chi_{m}, n\right)} \mathcal{K}_{\pi}(\underbrace{L_{b} Z_{\ell}, \ldots, L_{b} Z_{\ell}}_{m \text { entries }}, \underbrace{R_{d} Z_{r}, \ldots, R_{d} Z_{r}}_{n-1 \text { entries }}, R_{d} Z_{r} R_{c}) \\
& +\sum_{\substack{\pi \in \mathcal{B} \mathcal{N} \mathcal{C}\left(\chi_{m, n}\right) \\
\pi \notin \mathcal{B} \mathcal{N} \mathcal{C}_{\text {vs }}\left(\chi_{m, n}\right)}} \mathcal{K}_{\pi}(\underbrace{L_{b} Z_{\ell}, \ldots, L_{b} Z_{\ell}}_{m \text { entries }}, \underbrace{R_{d} Z_{r}, \ldots, R_{d} Z_{r}}_{n-1 \text { entries }}, R_{d} Z_{r} R_{c}) \\
& =\mathbb{F}\left(\left(L_{b} Z_{\ell}\right)^{m}\right) c \mathbb{F}\left(\left(R_{d} Z_{r}\right)^{n}\right)+\Theta_{m, n}(b, c, d),
\end{aligned}
$$

where $\Theta_{m, n}(b, c, d)$ denotes the sum

$$
\sum_{\substack{\pi \in \mathcal{B N \mathcal { C }}\left(\chi_{m, n}\right) \\ \pi \notin \mathcal{B N \mathcal { N }} \mathcal{C}_{\mathrm{vs}}\left(\chi_{m, n}\right)}} \mathcal{K}_{\pi}(\underbrace{L_{b} Z_{\ell}, \ldots, L_{b} Z_{\ell}}_{m \text { entries }}, \underbrace{R_{d} Z_{r}, \ldots, R_{d} Z_{r}}_{n-1 \text { entries }}, R_{d} Z_{r} R_{c}) .
$$

For every partition $\pi \in \mathcal{B N C}\left(\chi_{m, n}\right) \backslash \mathcal{B N C}_{\text {vs }}\left(\chi_{m, n}\right)$, let $V_{\pi}$ denote the block of $\pi$ with both left and right indices such that $\min \left(V_{\pi}\right)$ is the smallest among all blocks of $\pi$ with this property. Note that $V_{\pi}$ is necessarily an exterior block. Rearrange the sum in $\Theta_{m, n}(b, c, d)$ (which may be done as it converges absolutely) by first choosing $s \in\{1, \ldots, m\}, t \in\{1, \ldots, n\}, V \subset\{1, \ldots, m+n\}$ such that

$$
V_{\ell}:=V \cap\{1, \ldots, m\}=\left\{u_{1}<\cdots<u_{s}\right\} \quad \text { and } \quad V_{r}:=V \cap\{m+1, \ldots, m+n\}=\left\{v_{1}<\cdots<v_{t}\right\},
$$

and then summing over all $\pi \in \mathcal{B N C}\left(\chi_{m, n}\right) \backslash \mathcal{B N C}_{\mathrm{vs}}\left(\chi_{m, n}\right)$ such that $V_{\pi}=V$. The result is

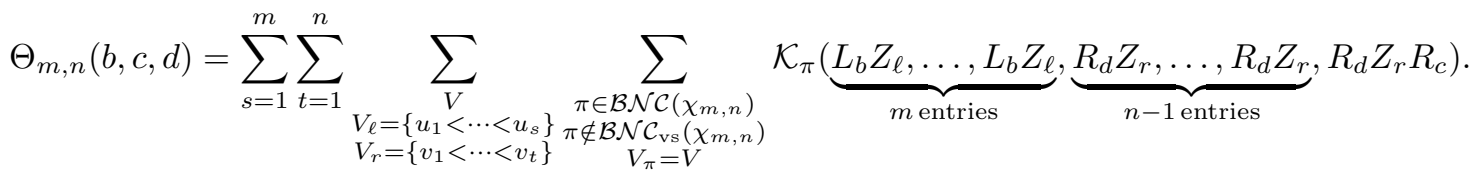

Using operator-valued conditionally bi-multiplicative properties, the right-most sum in the above expression is

$$
F_{b} \mathcal{K}_{1_{\chi_{s, t}}}\left(Z_{\ell}, L_{b_{2}} Z_{\ell}, \ldots, L_{b_{s}} Z_{\ell}, Z_{r}, R_{d_{2}} Z_{r}, \ldots, R_{d_{t-1}} Z_{r}, R_{d_{t}} Z_{r} R_{\mathbb{E}\left(\left(L_{b} Z_{\ell}\right)^{m-u_{s}}\left(R_{d} Z_{r}\right)^{n-v_{t}} R_{c}\right)}\right) G_{d}
$$

where

$$
\begin{gathered}
b_{k}=\mathbb{E}\left(\left(L_{b} Z_{\ell}\right)^{u_{k}-u_{k-1}-1}\right) b, \quad d_{k}=d \mathbb{E}\left(\left(R_{d} Z_{r}\right)^{v_{k}-v_{k-1}-1}\right), \\
F_{b}=\mathbb{F}\left(\left(L_{b} Z_{\ell}\right)^{u_{1}-1}\right) b, \quad \text { and } \quad G_{d}=d \mathbb{F}\left(\left(R_{d} Z_{r}\right)^{v_{1}-1}\right) .
\end{gathered}
$$

Consequently, we obtain that $\Theta_{m, n}(b, c, d)$ equals

$$
\sum_{\substack{1 \leq s \leq m \\ 0 \leq i_{0}, i_{1}, \ldots, i_{s} \leq m \\ i_{0}+i_{1}+\cdots+i_{s}=m-s}} \sum_{\substack{1 \leq t \leq n \\ 0 \leq j_{0}, j_{1}, \ldots, j_{t} \leq n \\ j_{1}+\cdots+j_{t}=n-t}} \sum_{\left.i_{1}\right) \mathcal{K}_{1_{\chi_{s, t}}}\left(Z_{\ell}, L_{f\left(i_{2}\right)} Z_{\ell}, \ldots, L_{f\left(i_{s}\right)} Z_{\ell}, Z_{r}, R_{g\left(j_{2}\right)} Z_{r}, \ldots, R_{g\left(j_{t}-1\right)} Z_{r}, R_{g\left(j_{t}\right)} Z_{r} R_{\mathbb{E}\left(\left(L_{b} Z_{\ell}\right)^{i_{0}}\left(R_{d} Z_{r}\right)^{j_{0}} R_{c}\right)}\right) G\left(j_{1}\right),}
$$

where

$$
f(k)=\mathbb{E}\left(\left(L_{b} Z_{\ell}\right)^{k}\right) b, \quad g(k)=d \mathbb{E}\left(\left(R_{d} Z_{r}\right)^{k}\right), \quad F(k)=\mathbb{F}\left(\left(L_{b} Z_{\ell}\right)^{k}\right) b, \quad \text { and } \quad G(k)=d \mathbb{F}\left(\left(R_{d} Z_{r}\right)^{k}\right) .
$$

Note that

$$
\sum_{k \geq 0} f(k)=M_{Z_{\ell}}^{\ell}(b) b, \quad \sum_{k \geq 0} g(k)=d M_{Z_{r}}^{r}(d), \quad \sum_{k \geq 0} F(k)=\mathbb{M}_{Z_{\ell}}^{\ell}(b) b, \quad \text { and } \quad \sum_{k \geq 0} G(k)=d \mathbb{M}_{Z_{r}}^{r}(d) .
$$


On the other hand, expanding $\mathbb{M}_{\left(Z_{\ell}, Z_{r}\right)}(b, c, d)$ using the fact everything converges absolutely produces

$$
\begin{aligned}
& \mathbb{M}_{\left(Z_{\ell}, Z_{r}\right)}(b, c, d) \\
& =c+\sum_{m \geq 1} \mathbb{F}\left(\left(L_{b} Z_{\ell}\right)^{m} R_{c}\right)+\sum_{n \geq 1} \mathbb{F}\left(\left(R_{d} Z_{r}\right)^{n} R_{c}\right)+\sum_{m, n \geq 1} \mathbb{F}\left(\left(L_{b} Z_{\ell}\right)^{m}\left(R_{d} Z_{r}\right)^{n} R_{c}\right) \\
& =c+\sum_{m \geq 1} \mathbb{F}\left(\left(L_{b} Z_{\ell}\right)^{m} R_{c}\right)+\sum_{n \geq 1} \mathbb{F}\left(\left(R_{d} Z_{r}\right)^{n} R_{c}\right)+\sum_{m, n \geq 1} \mathbb{F}\left(\left(L_{b} Z_{\ell}\right)^{m}\right) c \mathbb{F}\left(\left(R_{d} Z_{r}\right)^{n}\right)+\sum_{m, n \geq 1} \Theta_{m, n}(b, c, d) \\
& =\sum_{m, n \geq 0} \mathbb{F}\left(\left(L_{b} Z_{\ell}\right)^{m}\right) c \mathbb{F}\left(\left(R_{d} Z_{r}\right)^{n}\right)+\sum_{m, n \geq 1} \Theta_{m, n}(b, c, d) \\
& =\mathbb{M}_{Z_{\ell}}^{\ell}(b) c \mathbb{M}_{Z_{r}}^{r}(d)+\sum_{m, n \geq 1} \Theta_{m, n}(b, c, d) .
\end{aligned}
$$

By rearranging the remaining sum involving $\Theta_{m, n}(b, c, d)$ to sum over all fixed $s, t$ in equation (8), and by choosing $b, d$ sufficiently small so that $M_{Z_{\ell}}^{\ell}(b), M_{Z_{r}}^{r}(d), \mathbb{M}_{Z_{\ell}}^{\ell}(b)$, and $\mathbb{M}_{Z_{r}}^{r}(d)$ are invertible, we obtain

$$
\begin{aligned}
& \sum_{m, n \geq 1} \Theta_{m, n}(b, c, d) \\
& =\sum_{s, t \geq 1} \mathbb{M}_{Z_{\ell}}^{\ell}(b) b
\end{aligned}
$$

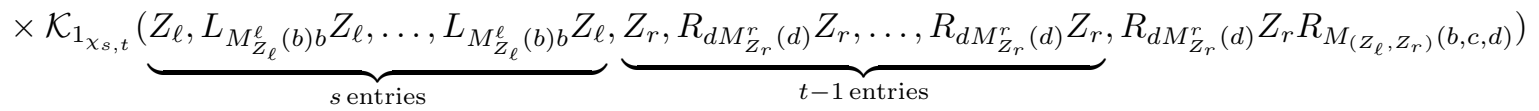

$$
\begin{aligned}
& \times d \mathbb{M}_{Z_{r}}^{r}(d) \\
& =\mathbb{M}_{Z_{\ell}}^{\ell}(b) M_{Z_{\ell}}^{\ell}(b)^{-1}
\end{aligned}
$$

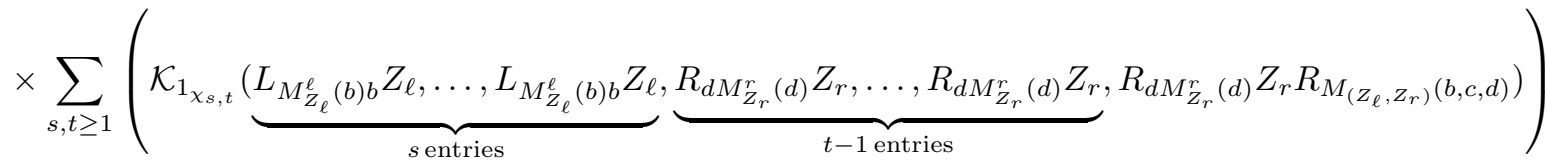

$$
\begin{aligned}
& \times M_{Z_{r}}^{r}(d)^{-1} \mathbb{M}_{Z_{r}}^{r}(d) \\
& =\mathbb{M}_{Z_{\ell}}^{\ell}(b) M_{Z_{\ell}}^{\ell}(b)^{-1}\left[\mathcal{C}_{\left(Z_{\ell}, Z_{r}\right)}\left(M_{Z_{\ell}}^{\ell}(b) b, M_{\left(Z_{\ell}, Z_{r}\right)}(b, c, d), d M_{Z_{r}}^{r}(d)\right)-\mathcal{C}_{Z_{\ell}}^{\ell}\left(M_{Z_{\ell}}^{\ell}(b) b\right) M_{\left(Z_{\ell}, Z_{r}\right)}(b, c, d)\right. \\
& \left.-M_{\left(Z_{\ell}, Z_{r}\right)}(b, c, d) \mathcal{C}_{Z_{r}}^{r}\left(d M_{Z_{r}}^{r}(d)\right)+M_{\left(Z_{\ell}, Z_{r}\right)}(b, c, d)\right] M_{Z_{r}}^{r}(d)^{-1} \mathbb{M}_{Z_{r}}^{r}(d) \\
& =\mathbb{M}_{Z_{\ell}}^{\ell}(b) M_{Z_{\ell}}^{\ell}(b)^{-1} \mathcal{C}_{\left(Z_{\ell}, Z_{r}\right)}\left(M_{Z_{\ell}}^{\ell}(b) b, M_{\left(Z_{\ell}, Z_{r}\right)}(b, c, d), d M_{Z_{r}}^{r}(d)\right) M_{Z_{r}}^{r}(d)^{-1} \mathbb{M}_{Z_{r}}^{r}(d) \\
& -\mathbb{M}_{Z_{\ell}}^{\ell}(b) M_{Z_{\ell}}^{\ell}(b)^{-1}\left(1+M_{Z_{\ell}}^{\ell}(b)-M_{Z_{\ell}}^{\ell}(b) \mathbb{M}_{Z_{\ell}}^{\ell}(b)^{-1}\right) M_{\left(Z_{\ell}, Z_{r}\right)}(b, c, d) M_{Z_{r}}^{r}(d)^{-1} \mathbb{M}_{Z_{r}}^{r}(d) \\
& -\mathbb{M}_{Z_{\ell}}^{\ell}(b) M_{Z_{\ell}}^{\ell}(b)^{-1} M_{\left(Z_{\ell}, Z_{r}\right)}(b, c, d)\left(1+M_{Z_{r}}^{r}(d)-\mathbb{M}_{Z_{r}}^{r}(d)^{-1} M_{Z_{r}}^{r}(d)\right) M_{Z_{r}}^{r}(d)^{-1} \mathbb{M}_{Z_{r}}^{r}(d) \\
& +\mathbb{M}_{Z_{\ell}}^{\ell}(b) M_{Z_{\ell}}^{\ell}(b)^{-1} M_{\left(Z_{\ell}, Z_{r}\right)}(b, c, d) M_{Z_{r}}^{r}(d)^{-1} \mathbb{M}_{Z_{r}}^{r}(d) \\
& =\mathbb{M}_{Z_{\ell}}^{\ell}(b) M_{Z_{\ell}}^{\ell}(b)^{-1}\left(\mathcal{C}_{\left(Z_{\ell}, Z_{r}\right)}\left(M_{Z_{\ell}}^{\ell}(b) b, M_{\left(Z_{\ell}, Z_{r}\right)}(b, c, d), d M_{Z_{r}}^{r}(d)\right)-M_{\left(Z_{\ell}, Z_{r}\right)}(b, c, d)\right) M_{Z_{r}}^{r}(d)^{-1} \mathbb{M}_{Z_{r}}^{r}(d) \\
& \text { - }\left(\mathbb{M}_{Z_{\ell}}^{\ell}(b)-1\right) M_{\left(Z_{\ell}, Z_{r}\right)}(b, c, d) M_{Z_{r}}^{r}(d)^{-1} \mathbb{M}_{Z_{r}}^{r}(d)-\mathbb{M}_{Z_{\ell}}^{\ell}(b) M_{Z_{\ell}}^{\ell}(b)^{-1} M_{\left(Z_{\ell}, Z_{r}\right)}(b, c, d)\left(\mathbb{M}_{Z_{r}}^{r}(d)-1\right),
\end{aligned}
$$

where the fourth equality follows from Lemma 8.2 . The result now follows by combining these equations.

\section{Operator-VAlued CONDitionAlly BI-FREE LiMit THEOREMS}

In this section, operator-valued conditionally bi-free limit theorems are studied. Recall first from Definition 6.2 that if $\mathcal{Z}=\left\{Z_{i}\right\}_{i \in I} \sqcup\left\{Z_{j}\right\}_{j \in J}$ is a two-faced family in a $\mathcal{B}$ - $\mathcal{B}$-non-commutative probability space with a pair of $(\mathcal{B}, \mathcal{D})$-valued expectations $(\mathcal{A}, \mathbb{E}, \mathbb{F}, \varepsilon)$, then the moment and cumulant series $\left(\nu^{\mathcal{Z}}, \mu^{\mathcal{Z}}\right)$ and $\left(\rho^{\mathcal{Z}}, \eta^{\mathcal{Z}}\right)$ completely describe the joint distribution of $\mathcal{Z}$ with respect to $(\mathbb{E}, \mathbb{F})$. In that which follows, given a bi-noncrossing partition $\pi \in \mathcal{B N C}(\chi)$ it is often convenient to define

$$
\left(\nu_{\omega}^{\mathcal{Z}}\right)_{\pi}\left(b_{1}, \ldots, b_{n-1}\right), \quad\left(\mu_{\omega}^{\mathcal{Z}}\right)_{\pi}\left(b_{1}, \ldots, b_{n-1}\right), \quad\left(\rho_{\omega}^{\mathcal{Z}}\right)_{\pi}\left(b_{1}, \ldots, b_{n-1}\right), \quad \text { and } \quad\left(\eta_{\omega}^{\mathcal{Z}}\right)_{\pi}\left(b_{1}, \ldots, b_{n-1}\right)
$$

by using operator-valued conditionally bi-multiplicativity and replacing $1_{\chi_{\omega}}$ with $\pi$ in Notation 6.1 . 
9.1. The operator-valued c-bi-free central limit theorem. Like any non-commutative probability theory, the first result is a central limit theorem in the operator-valued c-bi-free setting.

Definition 9.1. A two-faced family $\mathcal{Z}=\left(\left(Z_{i}\right)_{i \in I},\left(Z_{j}\right)_{j \in J}\right)$ in a $\mathcal{B}$ - $\mathcal{B}$-non-commutative probability space with a pair of $(\mathcal{B}, \mathcal{D})$-valued expectations $(\mathcal{A}, \mathbb{E}, \mathbb{F}, \varepsilon)$ is said to have a centred $(\mathcal{B}, \mathcal{D})$-valued $c$-bi-free Gaussian distribution if

$$
\rho_{\omega}^{\mathcal{Z}}\left(b_{1}, \ldots, b_{n-1}\right)=\eta_{\omega}^{\mathcal{Z}}\left(b_{1}, \ldots, b_{n-1}\right)=0
$$

for all $n \geq 1$ with $n \neq 2, \omega:\{1, \ldots, n\} \rightarrow I \sqcup J$, and $b_{1}, \ldots, b_{n-1} \in \mathcal{B}$.

In view of the definition above and the moment-cumulant formulae, it is enough to specify $\nu_{\omega}^{\mathcal{Z}}(b)$ and $\mu_{\omega}^{\mathcal{Z}}(b)$ for $\omega:\{1,2\} \rightarrow I \sqcup J$ and $b \in \mathcal{B}$.

Definition 9.2. Let $I$ and $J$ be non-empty disjoint finite sets, let $M_{|I \sqcup J|}(\mathcal{B})$ and $M_{|I \sqcup J|}(\mathcal{D})$ denote the $|I \sqcup J|$ by $|I \sqcup J|$ matrices with entries in $\mathcal{B}$ and $\mathcal{D}$ respectively, and let

$$
\sigma: \mathcal{B} \rightarrow M_{|I \sqcup J|}(\mathcal{B}), \quad b \mapsto\left(\sigma_{k, \ell}(b)\right)_{k, \ell \in I \sqcup J} \quad \text { and } \quad \tau: \mathcal{B} \rightarrow M_{|I \sqcup J|}(\mathcal{D}), \quad b \mapsto\left(\tau_{k, \ell}(b)\right)_{k, \ell \in I \sqcup J}
$$

be linear maps. A two-faced family $\mathcal{Z}=\left(\left(Z_{i}\right)_{i \in I},\left(Z_{j}\right)_{j \in J}\right)$ in a $\mathcal{B}$ - $\mathcal{B}$-non-commutative probability space with a pair of $(\mathcal{B}, \mathcal{D})$-valued expectations $(\mathcal{A}, \mathbb{E}, \mathbb{F}, \varepsilon)$ is said to have a centred $(\mathcal{B}, \mathcal{D})$-valued c-bi-free Gaussian distribution with covariance matrices $(\sigma, \tau)$ if, in addition to having a centred $(\mathcal{B}, \mathcal{D})$-valued c-bi-free Gaussian distribution,

$$
\nu_{\omega}^{\mathcal{Z}}(b)=\sigma_{\omega(1), \omega(2)}(b) \in \mathcal{B} \quad \text { and } \quad \mu_{\omega}^{\mathcal{Z}}(b)=\tau_{\omega(1), \omega(2)}(b) \in \mathcal{D}
$$

for all $\omega:\{1,2\} \rightarrow I \sqcup J$ and $b \in \mathcal{B}$.

Theorem 9.3. Let $\left\{\mathcal{Z}_{m}=\left(\left(Z_{m ; i}\right)_{i \in I},\left(Z_{m ; j}\right)_{j \in J}\right)\right\}_{m=1}^{\infty}$ be a sequence of two-faced families in a Banach $\mathcal{B}-\mathcal{B}$-non-commutative probability space $(\mathcal{A}, \mathbb{E}, \mathbb{F}, \varepsilon)$ which are $c$-bi-free over $(\mathcal{B}, \mathcal{D})$. Moreover assume

(1) $\mathbb{E}\left(Z_{m ; k}\right)=\mathbb{F}\left(Z_{m ; k}\right)=0$ for all $m \geq 1$ and $k \in I \sqcup J$;

(2) $\sup _{m>1}\left\|\nu_{\omega}^{\mathcal{Z}_{m}}\left(b_{1}, \ldots, b_{n-1}\right)\right\|<\infty$ and $\sup _{m>1}\left\|\mu_{\omega}^{\mathcal{Z}_{m}}\left(b_{1}, \ldots, b_{n-1}\right)\right\|<\infty$ for all $n \geq 1, \omega:\{1, \ldots, n\} \rightarrow$ $I \sqcup J$, and $b_{1}, \ldots, b_{n-1} \in \mathcal{B}$;

(3) there are linear maps $\sigma: \mathcal{B} \rightarrow M_{|I \sqcup J|}(\mathcal{B})$ and $\tau: \mathcal{B} \rightarrow M_{|I \sqcup J|}(\mathcal{D})$ such that

$$
\lim _{N \rightarrow \infty} \frac{1}{N} \sum_{m=1}^{N} \nu_{\omega}^{\mathcal{Z}_{m}}(b)=\sigma_{\omega(1), \omega(2)}(b) \quad \text { and } \quad \lim _{N \rightarrow \infty} \frac{1}{N} \sum_{m=1}^{N} \mu_{\omega}^{\mathcal{Z}_{m}}(b)=\tau_{\omega(1), \omega(2)}(b)
$$

for all $\omega:\{1,2\} \rightarrow I \sqcup J$ and $b \in \mathcal{B}$.

Then the two-faced families $\left\{\mathcal{S}_{N}=\left(\left(S_{N ; i}\right)_{i \in I},\left(S_{N ; j}\right)_{j \in J}\right)\right\}_{N=1}^{\infty}$, defined by

$$
S_{N ; k}=\frac{1}{\sqrt{N}} \sum_{m=1}^{N} Z_{m ; k}, \quad k \in I \sqcup J,
$$

converges in distribution to a two-faced family $\mathcal{Y}=\left(\left(Y_{i}\right)_{i \in I},\left(Y_{j}\right)_{j \in J}\right)$ which has a centred $(\mathcal{B}, \mathcal{D})$-valued c-bi-free Gaussian distribution with covariance matrices $(\sigma, \tau)$.

Proof. Since the cumulant series uniquely determine the joint distributions, it suffices to show that

$$
\lim _{N \rightarrow \infty} \rho_{\omega}^{\mathcal{S}_{N}}\left(b_{1}, \ldots, b_{n-1}\right)=\rho_{\omega}^{\mathcal{Y}}\left(b_{1}, \ldots, b_{n-1}\right) \quad \text { and } \lim _{N \rightarrow \infty} \eta_{\omega}^{\mathcal{S}_{N}}\left(b_{1}, \ldots, b_{n-1}\right)=\eta_{\omega}^{\mathcal{Y}}\left(b_{1}, \ldots, b_{n-1}\right)
$$

for all $n \geq 1, \omega:\{1, \ldots, n\} \rightarrow I \sqcup J$, and $b_{1}, \ldots, b_{n-1} \in \mathcal{B}$. By definitions, this means

$$
\lim _{N \rightarrow \infty} \rho_{\omega}^{\mathcal{S}_{N}}\left(b_{1}, \ldots, b_{n-1}\right)=\lim _{N \rightarrow \infty} \eta_{\omega}^{\mathcal{S}_{N}}\left(b_{1}, \ldots, b_{n-1}\right)=0
$$

for all $\omega:\{1, \ldots, n\} \rightarrow I \sqcup J$ such that $n \neq 2$,

$$
\lim _{N \rightarrow \infty} \rho_{\omega}^{\mathcal{S}_{N}}(b)=\sigma_{\omega(1), \omega(2)}(b) \quad \text { and } \quad \lim _{N \rightarrow \infty} \eta_{\omega}^{\mathcal{S}_{N}}(b)=\tau_{\omega(1), \omega(2)}(b)
$$

for all $\omega:\{1,2\} \rightarrow I \sqcup J$ and $b \in \mathcal{B}$. 
For fixed $n \geq 1, \omega:\{1, \ldots, n\} \rightarrow I \sqcup J$, and $b_{1}, \ldots, b_{n-1} \in \mathcal{B}$, by the additive and multilinear properties of cumulants, we have

$$
\begin{aligned}
& E:=\lim _{N \rightarrow \infty} \rho_{\omega}^{\mathcal{S}_{N}}\left(b_{1}, \ldots, b_{n-1}\right)=\lim _{N \rightarrow \infty} \frac{1}{N^{n / 2}} \sum_{m=1}^{N} \rho_{\omega}^{\mathcal{Z}_{m}}\left(b_{1}, \ldots, b_{n-1}\right), \\
& F:=\lim _{N \rightarrow \infty} \eta_{\omega}^{\mathcal{S}_{N}}\left(b_{1}, \ldots, b_{n-1}\right)=\lim _{N \rightarrow \infty} \frac{1}{N^{n / 2}} \sum_{m=1}^{N} \eta_{\omega}^{\mathcal{Z}_{m}}\left(b_{1}, \ldots, b_{n-1}\right) .
\end{aligned}
$$

If $n=1$, then

$$
\begin{aligned}
& E=\lim _{N \rightarrow \infty} \frac{1}{\sqrt{N}} \sum_{m=1}^{N} \mathbb{E}\left(Z_{m ; \omega(1)}\right)=0, \\
& F=\lim _{N \rightarrow \infty} \frac{1}{\sqrt{N}} \sum_{m=1}^{N} \mathbb{F}\left(Z_{m ; \omega(1)}\right)=0
\end{aligned}
$$

by assumption (1). If $n \geq 3$, then assumption (2) and operator-valued conditionally bi-multiplicativity imply

$$
\sup _{m \geq 1}\left\|\rho_{\omega}^{\mathcal{Z}_{m}}\left(b_{1}, \ldots, b_{n-1}\right)\right\|:=B<\infty \quad \text { and } \quad \sup _{m \geq 1}\left\|\eta_{\omega}^{\mathcal{Z}_{m}}\left(b_{1}, \ldots, b_{n-1}\right)\right\|:=D<\infty
$$

hence

$$
\|E\| \leq \lim _{N \rightarrow \infty} \frac{B}{N^{(n-2) / 2}}=0 \quad \text { and } \quad\|F\| \leq \lim _{N \rightarrow \infty} \frac{D}{N^{(n-2) / 2}}=0 .
$$

Otherwise $n=2$ and

$$
E=\lim _{N \rightarrow \infty} \frac{1}{N} \sum_{m=1}^{N} \rho_{\omega}^{\mathcal{Z}_{m}}(b)=\lim _{N \rightarrow \infty} \frac{1}{N} \sum_{m=1}^{N} \nu_{\omega}^{\mathcal{Z}_{m}}(b)=\sigma_{\omega(1), \omega(2)}(b),
$$

and similarly $F=\tau_{\omega(1), \omega(2)}(b)$, for all $\omega:\{1,2\} \rightarrow I \sqcup J$ and $b \in \mathcal{B}$ by assumptions (1) and (3).

9.2. The operator-valued compound c-bi-free Poisson limit theorem. The next result is a Poisson type limit theorem in the operator-valued c-bi-free setting. In what follows, all two-faced families are assumed to have non-empty disjoint left and right index sets $I$ and $J$, respectively. To formulate the statement, we introduce the following notation.

Let $\left(\nu_{1}, \mu_{1}\right)$ and $\left(\nu_{2}, \mu_{2}\right)$ be the moment series of two-faced families. For $\lambda \in \mathbb{R}$, denote by

$$
\left(\lambda \nu_{1}+(1-\lambda) \nu_{2}, \lambda \mu_{1}+(1-\lambda) \mu_{2}\right)
$$

the moment series of some two-faced family such that

$$
\left(\lambda \nu_{1}+(1-\lambda) \nu_{2}\right)_{\omega}\left(b_{1}, \ldots, b_{n-1}\right)=\lambda\left(\nu_{1}\right)_{\omega}\left(b_{1}, \ldots, b_{n-1}\right)+(1-\lambda)\left(\nu_{2}\right)_{\omega}\left(b_{1}, \ldots, b_{n-1}\right)
$$

and

$$
\left(\lambda \mu_{1}+(1-\lambda) \mu_{2}\right)_{\omega}\left(b_{1}, \ldots, b_{n-1}\right)=\lambda\left(\mu_{1}\right)_{\omega}\left(b_{1}, \ldots, b_{n-1}\right)+(1-\lambda)\left(\mu_{2}\right)_{\omega}\left(b_{1}, \ldots, b_{n-1}\right)
$$

for all $n \geq 1, \omega:\{1, \ldots, n\} \rightarrow I \sqcup J$, and $b_{1}, \ldots, b_{n-1} \in \mathcal{B}$. Such a realization always exists by similar (and simpler) constructions as in the proofs of [11, Lemma 3.8] and Lemma 6.3. Moreover, let $\left(\nu^{\delta}, \mu^{\delta}\right)$ be the special moment series such that

$$
\nu_{\omega}^{\delta}\left(b_{1}, \ldots, b_{n-1}\right)=\mu_{\omega}^{\delta}\left(b_{1}, \ldots, b_{n-1}\right)=0
$$

for all $n \geq 1, \omega:\{1, \ldots, n\} \rightarrow I \sqcup J$, and $b_{1}, \ldots, b_{n-1} \in \mathcal{B}$.

Definition 9.4. Let $(\nu, \mu)$ be the moment series of some two-faced family and let $\lambda \in \mathbb{R}$. A two-faced family $\mathcal{Z}=\left(\left(Z_{i}\right)_{i \in I},\left(Z_{j}\right)_{j \in J}\right)$ in a $\mathcal{B}$ - $\mathcal{B}$-non-commutative probability space with a pair of $(\mathcal{B}, \mathcal{D})$-valued expectations $(\mathcal{A}, \mathbb{E}, \mathbb{F}, \varepsilon)$ is said to have a $(\mathcal{B}, \mathcal{D})$-valued compound $c$-bi-free Poisson distribution with rate $\lambda$ and jump distribution $(\nu, \mu)$ if

$$
\rho_{\omega}^{\mathcal{Z}}\left(b_{1}, \ldots, b_{n-1}\right)=\lambda \nu_{\omega}\left(b_{1}, \ldots, b_{n-1}\right) \quad \text { and } \quad \eta_{\omega}^{\mathcal{Z}}\left(b_{1}, \ldots, b_{n-1}\right)=\lambda \mu_{\omega}\left(b_{1}, \ldots, b_{n-1}\right)
$$

for all $n \geq 1, \omega:\{1, \ldots, n\} \rightarrow I \sqcup J$, and $b_{1}, \ldots, b_{n-1} \in \mathcal{B}$. 
Theorem 9.5. Let $(\nu, \mu)$ be the moment series of some two-faced family, let $\lambda \in \mathbb{R}$, and consider the sequence $\left\{\left(\nu_{N}, \mu_{N}\right)\right\}_{N=1}^{\infty}$ of moment series defined by

$$
\nu_{N}=\left(1-\frac{\lambda}{N}\right) \nu^{\delta}+\frac{\lambda}{N} \nu \quad \text { and } \quad \mu_{N}=\left(1-\frac{\lambda}{N}\right) \mu^{\delta}+\frac{\lambda}{N} \mu .
$$

If $\left\{\mathcal{Z}_{N ; m}=\left(\left(Z_{N ; m ; i}\right)_{i \in I},\left(Z_{N ; m ; j}\right)_{j \in J}\right)\right\}_{m=1}^{N}$ is a sequence of identically distributed two-faced families in a $\mathcal{B}-\mathcal{B}$-non-commutative probability space with a pair of $(\mathcal{B}, \mathcal{D})$-valued expectations $(\mathcal{A}, \mathbb{E}, \mathbb{F}, \varepsilon)$ which are c-bifree over $(\mathcal{B}, \mathcal{D})$ with moment series $\left(\nu_{N}, \mu_{N}\right)$, then the two-faced families $\left\{\mathcal{S}_{N}=\left(\left(S_{N ; i}\right)_{i \in I},\left(S_{N ; j}\right)_{j \in J}\right)\right\}_{N=1}^{\infty}$, defined by

$$
S_{N ; k}=\sum_{m=1}^{N} Z_{N ; m ; k}, \quad k \in I \sqcup J,
$$

converges in distribution to a two-faced family $\mathcal{Z}=\left(\left(Z_{i}\right)_{i \in I},\left(Z_{j}\right)_{j \in J}\right)$ which has a $(\mathcal{B}, \mathcal{D})$-valued compound $c$-bi-free Poisson distribution with rate $\lambda$ and jump distribution $(\nu, \mu)$.

Proof. For each $N \geq 1$, let $\left(\rho_{N}, \eta_{N}\right)$ be the cumulant series corresponding to $\left(\nu_{N}, \mu_{N}\right)$. For $n \geq 1, \omega$ : $\{1, \ldots, n\} \rightarrow I \sqcup J$, and $b_{1}, \ldots, b_{n-1} \in \mathcal{B}$, we have

$$
\begin{aligned}
\left(\rho_{N}\right)_{\omega}\left(b_{1}, \ldots, b_{n-1}\right) & =\sum_{\pi \in \mathcal{B N \mathcal { C }}\left(\chi_{\omega}\right)}\left(\nu_{N}\right)_{\pi}\left(b_{1}, \ldots, b_{n-1}\right) \mu_{\mathcal{B N C} \mathcal{C}}\left(\pi, 1_{\chi_{\omega}}\right) \\
& =\left(\nu_{N}\right)_{\omega}\left(b_{1}, \ldots, b_{n-1}\right)+O\left(1 / N^{2}\right) \\
& =\frac{\lambda}{N} \nu_{\omega}\left(b_{1}, \ldots, b_{n-1}\right)+O\left(1 / N^{2}\right)
\end{aligned}
$$

and thus

$$
\begin{aligned}
\rho_{\omega}^{\mathcal{Z}}\left(b_{1}, \ldots, b_{n-1}\right) & =\lim _{N \rightarrow \infty} \rho_{\omega}^{\mathcal{S}_{N}}\left(b_{1}, \ldots, b_{n-1}\right) \\
& =\lim _{N \rightarrow \infty}\left(\lambda \nu_{\omega}\left(b_{1}, \ldots, b_{n-1}\right)+O(1 / N)\right) \\
& =\lambda \nu_{\omega}\left(b_{1}, \ldots, b_{n-1}\right) .
\end{aligned}
$$

Similarly, we have $\left(\eta_{N}\right)_{\pi}\left(b_{1}, \ldots, b_{n-1}\right)=O\left(1 / N^{2}\right)$ for $\pi \in \mathcal{B N C}\left(\chi_{\omega}\right)$ with at least two blocks, therefore

$$
\left(\eta_{N}\right)_{\omega}\left(b_{1}, \ldots, b_{n-1}\right)=\left(\mu_{N}\right)_{\omega}\left(b_{1}, \ldots, b_{n-1}\right)+O\left(1 / N^{2}\right)=\frac{\lambda}{N} \mu_{\omega}\left(b_{1}, \ldots, b_{n-1}\right)+O\left(1 / N^{2}\right),
$$

and thus

$$
\eta_{\omega}^{\mathcal{Z}}\left(b_{1}, \ldots, b_{n-1}\right)=\lim _{N \rightarrow \infty} \eta_{\omega}^{\mathcal{S}_{N}}\left(b_{1}, \ldots, b_{n-1}\right)=\lambda \mu_{\omega}\left(b_{1}, \ldots, b_{n-1}\right)
$$

as required.

9.3. A general operator-valued c-bi-free limit theorem. We finish this section with an operator-valued analogue of [6, Theorem 6.8].

Lemma 9.6. For every $N \in \mathbb{N}$, let $\mathcal{Z}_{N}=\left(\left(Z_{N ; i}\right)_{i \in I},\left(Z_{N ; j}\right)_{j \in J}\right)$ be a two-faced family in a Banach $\mathcal{B}$-B-non-

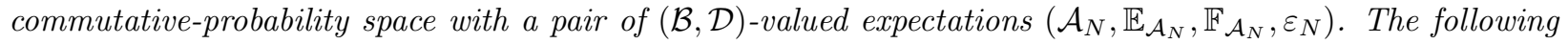
assertions are equivalent.

(1) For all $n \geq 1, \omega:\{1, \ldots, n\} \rightarrow I \sqcup J$, and $b_{1}, \ldots, b_{n-1} \in \mathcal{B}$, the limits

$$
\lim _{N \rightarrow \infty} N \nu_{\omega}^{\mathcal{Z}_{n}}\left(b_{1}, \ldots, b_{n-1}\right) \in \mathcal{B} \quad \text { and } \quad \lim _{N \rightarrow \infty} N \mu_{\omega}^{\mathcal{Z}_{n}}\left(b_{1}, \ldots, b_{n-1}\right) \in \mathcal{D}
$$

exist.

(2) For all $n \geq 1, \omega:\{1, \ldots, n\} \rightarrow I \sqcup J$, and $b_{1}, \ldots, b_{n-1} \in \mathcal{B}$, the limits

$$
\lim _{N \rightarrow \infty} N \rho_{\omega}^{\mathcal{Z}_{n}}\left(b_{1}, \ldots, b_{n-1}\right) \in \mathcal{B} \quad \text { and } \quad \lim _{N \rightarrow \infty} N \eta_{\omega}^{\mathcal{Z}_{n}}\left(b_{1}, \ldots, b_{n-1}\right) \in \mathcal{D}
$$

exist. 
Moreover, if these assertions hold, then

$$
\begin{aligned}
\lim _{N \rightarrow \infty} N \nu_{\omega}^{\mathcal{Z}_{n}}\left(b_{1}, \ldots, b_{n-1}\right) & =\lim _{N \rightarrow \infty} N \rho_{\omega}^{\mathcal{Z}_{n}}\left(b_{1}, \ldots, b_{n-1}\right) \quad \text { and } \\
\lim _{N \rightarrow \infty} N \mu_{\omega}^{\mathcal{Z}_{n}}\left(b_{1}, \ldots, b_{n-1}\right) & =\lim _{N \rightarrow \infty} N \eta_{\omega}^{\mathcal{Z}_{n}}\left(b_{1}, \ldots, b_{n-1}\right)
\end{aligned}
$$

for all $n \geq 1, \omega:\{1, \ldots, n\} \rightarrow I \sqcup J$, and $b_{1}, \ldots, b_{n-1} \in \mathcal{B}$.

Proof. Suppose assertion (2) holds. Since $\left(\kappa_{\mathcal{A}_{N}}, \mathcal{K}_{\mathcal{A}_{N}}\right)$ is operator-valued conditionally bi-multiplicative, we have

$$
\left(\rho_{\omega}^{\mathcal{Z}_{n}}\right)_{\pi}\left(b_{1}, \ldots, b_{n-1}\right)=O\left(1 / N^{2}\right) \quad \text { and } \quad\left(\eta_{\omega}^{\mathcal{Z}_{n}}\right)_{\pi}\left(b_{1}, \ldots, b_{n-1}\right)=O\left(1 / N^{2}\right)
$$

for $\pi \in \mathcal{B N C}\left(\chi_{\omega}\right)$ with at least two blocks. Hence

$$
\begin{aligned}
\lim _{N \rightarrow \infty} N \nu_{\omega}^{\mathcal{Z}_{n}}\left(b_{1}, \ldots, b_{n-1}\right) & =\lim _{N \rightarrow \infty} N \sum_{\pi \in \mathcal{B N C}\left(\chi_{\omega}\right)}\left(\rho_{\omega}^{\mathcal{Z}_{n}}\right)_{\pi}\left(b_{1}, \ldots, b_{n-1}\right) \\
& =\lim _{N \rightarrow \infty}\left(N \rho_{\omega}^{\mathcal{Z}_{n}}\left(b_{1}, \ldots, b_{n-1}\right)+O(1 / N)\right),
\end{aligned}
$$

and similarly

$$
\lim _{N \rightarrow \infty} N \mu_{\omega}^{\mathcal{Z}_{n}}\left(b_{1}, \ldots, b_{n-1}\right)=\lim _{N \rightarrow \infty}\left(N \eta_{\omega}^{\mathcal{Z}_{n}}\left(b_{1}, \ldots, b_{n-1}\right)+O(1 / N)\right)
$$

for all $n \geq 1, \omega:\{1, \ldots, n\} \rightarrow I \sqcup J$, and $b_{1}, \ldots, b_{n-1} \in \mathcal{B}$.

The proof for the other direction is analogous by the operator-valued conditionally bi-multiplicativity of $\left(\mathcal{E}_{\mathcal{A}_{N}}, \mathcal{F}_{\mathcal{A}_{N}}\right)$ and the moment-cumulant formulae from Definitions 2.7 and 4.7

Theorem 9.7. For every $N \in \mathbb{N}$, let $\left(\mathcal{A}_{N}, \mathbb{E}_{N}, \mathbb{F}_{N}, \varepsilon_{N}\right)$ be a Banach $\mathcal{B}$ - $\mathcal{B}$-non-commutative-probability space with a pair of $(\mathcal{B}, \mathcal{D})$-valued expectations and let $\left\{\mathcal{Z}_{N ; m}=\left(\left(Z_{N ; m ; i}\right)_{i \in I},\left(Z_{N ; m ; j}\right)_{j \in J}\right)\right\}_{m=1}^{N}$ be a sequence of identically distributed two-faced families in $\left(\mathcal{A}_{N}, \mathbb{E}_{N}, \mathbb{F}_{N}, \varepsilon_{N}\right)$ which are c-bi-free over $(\mathcal{B}, \mathcal{D})$. Furthermore, let $\mathcal{S}_{N}=\left(\left(S_{N ; i}\right)_{i \in I},\left(S_{N ; j}\right)_{j \in J}\right)$ be the two-faced family in $\left(\mathcal{A}_{N}, \mathbb{E}_{N}, \mathbb{F}_{N}, \varepsilon_{N}\right)$ defined by

$$
S_{N ; k}=\sum_{m=1}^{N} Z_{N ; m ; k}, \quad k \in I \sqcup J .
$$

The following assertions are equivalent.

(1) There exists a two-faced family $\mathcal{Y}=\left(\left(Y_{i}\right)_{i \in I},\left(Y_{j}\right)_{j \in J}\right)$ in a Banach $\mathcal{B}$ - $\mathcal{B}$-non-commutative-probability space with a pair of $(\mathcal{B}, \mathcal{D})$-valued expectations $(\mathcal{A}, \mathbb{E}, \mathbb{F}, \varepsilon)$ such that $\mathcal{S}_{N}$ converges in distribution to $\mathcal{Y}$ as $N \rightarrow \infty$.

(2) For all $n \geq 1, \omega:\{1, \ldots, n\} \rightarrow I \sqcup J$, and $b_{1}, \ldots, b_{n-1} \in \mathcal{B}$, the limits

$$
\lim _{N \rightarrow \infty} N \nu_{\omega}^{\mathcal{Z}_{N ; m}}\left(b_{1}, \ldots, b_{n-1}\right) \quad \text { and } \quad \lim _{N \rightarrow \infty} N \mu_{\omega}^{\mathcal{Z}_{N ; m}}\left(b_{1}, \ldots, b_{n-1}\right)
$$

exist and are independent of $m$.

Moreover, if these assertions hold, then the operator-valued bi-free and conditionally bi-free cumulants of $\mathcal{Y}$ are given by

$$
\rho_{\omega}^{\mathcal{Y}}\left(b_{1}, \ldots, b_{n-1}\right)=\lim _{N \rightarrow \infty} N \nu_{\omega}^{\mathcal{Z}_{N ; m}}\left(b_{1}, \ldots, b_{n-1}\right) \quad \text { and } \quad \eta_{\omega}^{\mathcal{Y}}\left(b_{1}, \ldots, b_{n-1}\right)=\lim _{N \rightarrow \infty} N \mu_{\omega}^{\mathcal{Z}_{N ; m}}\left(b_{1}, \ldots, b_{n-1}\right)
$$

for all $n \geq 1, \omega:\{1, \ldots, n\} \rightarrow I \sqcup J$, and $b_{1}, \ldots, b_{n-1} \in \mathcal{B}$. 
Proof. Suppose assertion (1) holds. For $n \geq 1, \omega:\{1, \ldots, n\} \rightarrow I \sqcup J$, and $b_{1}, \ldots, b_{n-1} \in \mathcal{B}$, we have

$$
\begin{aligned}
& \nu_{\omega}^{\mathcal{Y}}\left(b_{1}, \ldots, b_{n-1}\right) \\
& =\lim _{N \rightarrow \infty} \nu_{\omega}^{\mathcal{S}_{N}}\left(b_{1}, \ldots, b_{n-1}\right) \\
& =\lim _{N \rightarrow \infty}\left(\mathcal{E}_{\mathcal{A}_{N}}\right)_{1_{\chi \omega}}\left(S_{N ; \omega(1)}, C_{b_{1}}^{\omega(2)} S_{N ; \omega(2)}, \ldots, C_{b_{n-2}}^{\omega(n)} S_{N ; \omega(n)} C_{b_{n-1}}^{\omega(n)}\right) \\
& =\lim _{N \rightarrow \infty} \sum_{t(1), \ldots, t(n)=1}^{N}\left(\mathcal{E}_{\mathcal{A}_{N}}\right)_{1_{\chi_{\omega}}}\left(Z_{N ; t(1) ; \omega(1)}, C_{b_{1}}^{\omega(2)} Z_{N ; t(2) ; \omega(2)}, \ldots, C_{b_{n-2}}^{\omega(n)} Z_{N ; t(n) ; \omega(n)} C_{b_{n-1}}^{\omega(n)}\right) \\
& =\lim _{N \rightarrow \infty} \sum_{t(1), \ldots, t(n)=1}^{N} \sum_{\pi \in \mathcal{B N \mathcal { C }}\left(\chi_{\omega}\right)}\left(\kappa_{\mathcal{A}_{N}}\right)_{\pi}\left(Z_{N ; t(1) ; \omega(1)}, C_{b_{1}}^{\omega(2)} Z_{N ; t(2) ; \omega(2)}, \ldots, C_{b_{n-2}}^{\omega(n)} Z_{N ; t(n) ; \omega(n)} C_{b_{n-1}}^{\omega(n)}\right) \\
& =\lim _{N \rightarrow \infty} \sum_{\pi \in \mathcal{B} \mathcal{N C}\left(\chi_{\omega}\right)} N^{|\pi|}\left(\kappa_{\mathcal{A}_{N}}\right)_{\pi}\left(Z_{N ; m ; \omega(1)}, C_{b_{1}}^{\omega(2)} Z_{N ; m ; \omega(2)}, \ldots, C_{b_{n-2}}^{\omega(n)} Z_{N ; m ; \omega(n)} C_{b_{n-1}}^{\omega(n)}\right) \\
& =\lim _{N \rightarrow \infty} \sum_{\pi \in \mathcal{B N C}\left(\chi_{\omega}\right)} N^{|\pi|}\left(\rho_{\omega}^{\mathcal{Z}_{N ; m}}\right)_{\pi}\left(b_{1}, \ldots, b_{n-1}\right),
\end{aligned}
$$

where the second equality follows from Notation 6.1 by assuming $\{\omega(k)\}_{k=1}^{n}$ intersects both $I$ and $J$ (the special cases that $\omega(k) \in I$ or $\omega(k) \in J$ for all $k$ can be checked similarly), and the fifth equality, which is independent of $m$, follows from the assumptions of c-bi-free independence over $(\mathcal{B}, \mathcal{D})$ and identical distribution. Since $\nu_{\omega}^{\mathcal{Y}}\left(b_{1}, \ldots, b_{n-1}\right)$ exist for all $n \geq 1$ and $\omega:\{1, \ldots, n\} \rightarrow I \sqcup J$, it can be shown by induction on $n$ that the limits

$$
\lim _{N \rightarrow \infty} N^{|\pi|}\left(\rho_{\omega}^{\mathcal{Z}_{N ; m}}\right)_{\pi}\left(b_{1}, \ldots, b_{n-1}\right)
$$

exist for all $n \geq 1, \omega:\{1, \ldots, n\} \rightarrow I \sqcup J, \pi \in \mathcal{B N C}\left(\chi_{\omega}\right)$, and $b_{1}, \ldots, b_{n-1} \in \mathcal{B}$. Indeed, the base case $n=1$ follows from the assumption that

$$
\lim _{N \rightarrow \infty} N\left(\kappa_{\mathcal{A}_{n}}\right)_{1_{\chi \omega}}\left(Z_{N ; m ; \omega(1)}\right)=\lim _{N \rightarrow \infty}\left(\kappa_{\mathcal{A}_{N}}\right)_{1_{\chi \omega}}\left(S_{N ; \omega(1)}\right)=\lim _{N \rightarrow \infty}\left(\mathcal{E}_{\mathcal{A}_{N}}\right)_{1_{\chi \omega}}\left(S_{N ; \omega(1)}\right)=\mathcal{E}_{1_{\chi \omega}}\left(Y_{\omega(1)}\right)
$$

exist for all $\omega:\{1\} \rightarrow I \sqcup J$. For the inductive step, the limit

$$
\nu_{\omega}^{\mathcal{Y}}\left(b_{1}, \ldots, b_{n-1}\right)
$$

exists by assumption, and the limit

$$
\lim _{N \rightarrow \infty} \sum_{\substack{\pi \in \mathcal{B} \mathcal{N} \mathcal{C}\left(\chi_{\omega}\right) \\ \pi \neq \chi_{\chi_{\omega}}}} N^{|\pi|}\left(\rho_{\omega}^{\mathcal{Z}_{N ; m}}\right)_{\pi}\left(b_{1}, \ldots, b_{n-1}\right)
$$

exists by induction hypothesis with operator-valued conditionally bi-multiplicativity, thus the limit

$$
\lim _{N \rightarrow \infty} N \rho_{\omega}^{\mathcal{Z}_{N ; m}}\left(b_{1}, \ldots, b_{n-1}\right)
$$

exists, and equals

$$
\lim _{N \rightarrow \infty} N \nu_{\omega}^{\mathcal{Z}_{N ; m}}\left(b_{1}, \ldots, b_{n-1}\right)
$$

by Lemma 9.6. On the other hand, it follows from a similar calculation as above that

$$
\begin{aligned}
\mu_{\omega}^{\mathcal{Y}}\left(b_{1}, \ldots, b_{n-1}\right) & =\lim _{N \rightarrow \infty} \mu_{\omega}^{\mathcal{S}_{N}}\left(b_{1}, \ldots, b_{n-1}\right) \\
& =\lim _{N \rightarrow \infty} \sum_{\pi \in \mathcal{B \mathcal { N C }}\left(\chi_{\omega}\right)} N^{|\pi|}\left(\eta_{\omega}^{\mathcal{Z}_{N ; m}}\right)_{\pi}\left(b_{1}, \ldots, b_{n-1}\right)
\end{aligned}
$$

for all $n \geq 1, \omega:\{1, \ldots, n\} \rightarrow I \sqcup J$, and $b_{1}, \ldots, b_{n-1} \in \mathcal{B}$, and a similar induction argument on $n$ shows that the limits

$$
\lim _{N \rightarrow \infty} N^{|\pi|}\left(\eta_{\omega}^{\mathcal{Z}_{N ; m}}\right)_{\pi}\left(b_{1}, \ldots, b_{n-1}\right)
$$

exist for all $n \geq 1, \omega:\{1, \ldots, n\} \rightarrow I \sqcup J, \pi \in \mathcal{B N C}\left(\chi_{\omega}\right)$, and $b_{1}, \ldots, b_{n-1} \in \mathcal{B}$. In particular, choose $\pi=1_{\chi_{\omega}}$ and apply Lemma 9.6, we obtain the existence of the limit

$$
\lim _{N \rightarrow \infty} N \mu_{\omega}^{\mathcal{Z}_{N ; m}}\left(b_{1}, \ldots, b_{n-1}\right) .
$$


Conversely, suppose assertion (2) holds. By Lemma 9.6 and operator-valued conditionally bi-multiplicativity, the limits

$$
\lim _{N \rightarrow \infty} N^{|\pi|}\left(\rho_{\omega}^{\mathcal{Z}_{N ; m}}\right)_{\pi}\left(b_{1}, \ldots, b_{n-1}\right) \quad \text { and } \quad \lim _{N \rightarrow \infty} N^{|\pi|}\left(\eta_{\omega}^{\mathcal{Z}_{N ; m}}\right)_{\pi}\left(b_{1}, \ldots, b_{n-1}\right)
$$

exist for all $n \geq 1, \omega:\{1, \ldots, n\} \rightarrow I \sqcup J, \pi \in \mathcal{B N C}\left(\chi_{\omega}\right)$, and $b_{1}, \ldots, b_{n-1} \in \mathcal{B}$. Therefore, by the calculations above,

$$
\lim _{N \rightarrow \infty} \nu_{\omega}^{\mathcal{S}_{N}}\left(b_{1}, \ldots, b_{n-1}\right)=\sum_{\pi \in \mathcal{B N \mathcal { C }}\left(\chi_{\omega}\right)} \lim _{N \rightarrow \infty} N^{|\pi|}\left(\rho_{\omega}^{\mathcal{Z}_{N ; m}}\right)_{\pi}\left(b_{1}, \ldots, b_{n-1}\right)
$$

and

$$
\lim _{N \rightarrow \infty} \mu_{\omega}^{\mathcal{S}_{N}}\left(b_{1}, \ldots, b_{n-1}\right)=\sum_{\pi \in \mathcal{B} \mathcal{N C}\left(\chi_{\omega}\right)} \lim _{N \rightarrow \infty} N^{|\pi|}\left(\eta_{\omega}^{\mathcal{Z}_{N ; m}}\right)_{\pi}\left(b_{1}, \ldots, b_{n-1}\right)
$$

and hence these limits exist. One concludes assertion (1) by using Lemma 6.3 to construct a two-faced family $\mathcal{Y}=\left(\left(Y_{i}\right)_{i \in I},\left(Y_{j}\right)_{j \in J}\right)$ in a Banach $\mathcal{B}$ - $\mathcal{B}$-non-commutative-probability space with a pair of $(\mathcal{B}, \mathcal{D})$ valued expectations $(\mathcal{A}, \mathbb{E}, \mathbb{F}, \varepsilon)$ and define $\nu_{\omega}^{\mathcal{Y}}\left(b_{1}, \ldots, b_{n-1}\right)$ and $\mu_{\omega}^{\mathcal{Y}}\left(b_{1}, \ldots, b_{n-1}\right)$ to be the corresponding limit in equations (9) and (10) respectively.

Finally, for $n \geq 1, \omega:\{1, \ldots, n\} \rightarrow I \sqcup J$, and $b_{1}, \ldots, b_{n-1} \in \mathcal{B}$, we have

$$
\begin{aligned}
\sum_{\pi \in \mathcal{B} \mathcal{N C}\left(\chi_{\omega}\right)}\left(\rho_{\omega}^{\mathcal{Y}}\right)_{\pi}\left(b_{1}, \ldots, b_{n-1}\right) & =\nu_{\omega}^{\mathcal{Y}}\left(b_{1}, \ldots, b_{n-1}\right) \\
& =\lim _{N \rightarrow \infty} \nu_{\omega}^{\mathcal{S}_{N}}\left(b_{1}, \ldots, b_{n-1}\right) \\
& =\sum_{\pi \in \mathcal{B} \mathcal{N C}\left(\chi_{\omega}\right)} \lim _{N \rightarrow \infty} N^{|\pi|}\left(\rho_{\omega}^{\mathcal{Z}_{N ; m}}\right)_{\pi}\left(b_{1}, \ldots, b_{n-1}\right),
\end{aligned}
$$

and similarly

$$
\sum_{\pi \in \mathcal{B N \mathcal { C }}\left(\chi_{\omega}\right)}\left(\eta_{\omega}^{\mathcal{Y}}\right)_{\pi}\left(b_{1}, \ldots, b_{n-1}\right)=\sum_{\pi \in \mathcal{B N C}\left(\chi_{\omega}\right)} \lim _{N \rightarrow \infty} N^{|\pi|}\left(\eta_{\omega}^{\mathcal{Z}_{N ; m}}\right)_{\pi}\left(b_{1}, \ldots, b_{n-1}\right) .
$$

A similar induction argument on $n$ shows that

$$
\begin{aligned}
& \left(\rho_{\omega}^{\mathcal{Y}}\right)_{\pi}\left(b_{1}, \ldots, b_{n-1}\right)=\lim _{N \rightarrow \infty} N^{|\pi|}\left(\rho_{\omega}^{\mathcal{Z}_{N ; m}}\right)_{\pi}\left(b_{1}, \ldots, b_{n-1}\right) \quad \text { and } \\
& \left(\eta_{\omega}^{\mathcal{Y}}\right)_{\pi}\left(b_{1}, \ldots, b_{n-1}\right)=\lim _{N \rightarrow \infty} N^{|\pi|}\left(\eta_{\omega}^{\mathcal{Z}_{N ; m}}\right)_{\pi}\left(b_{1}, \ldots, b_{n-1}\right)
\end{aligned}
$$

for all $\pi \in \mathcal{B N C}\left(\chi_{\omega}\right)$, from which the last claims follow from Lemma 9.6 applied to $\pi=1_{\chi_{\omega}}$.

\section{REFERENCES}

[1] S. Belinschi, M. Popa, and V. Vinnikov, Infinite divisibility and a non-commutative Boolean-to-free BercoviciPata bijection, J. Funct. Anal. 262 (2012), no. 1, 94-123.

[2] F. Boca, Free products of completely positive maps and spectral sets, J. Funct. Anal. 97 (1991), no. 2, 251-263.

[3] M. Bożejko, M. Leinert, and R. Speicher, Convolution and limit theorems for conditionally free random variables, Pacific J. Math. 175 (1996), no. 2, 357-388.

[4] I. Charlesworth, B. Nelson, and P. Skoufranis, Combinatorics of Bi-Freeness with Amalgamation, Comm. Math. Phys. 338 (2015), no. 2, 801-847.

[5] I. Charlesworth, B. Nelson, and P. Skoufranis, On two-faced families of non-commutative random variables, Canad. J. Math. 67 (2015), no. 6, 1290-1325.

[6] Y. Gu and P. Skoufranis, Conditionally bi-free independence for pairs of algebras (2016), 44 pp., available at arXiv: 1609.07475

[7] M. Mastnak and A. Nica, Double-ended queues and joint moments of left-right canonical operators on full Fock space, Int. J. Math. 26 (2015), no. 2, 1550016.

[8] W. Młotkowski, Operator-valued version of conditionally free product, Studia Math. 153 (2002), no. 1, 13-30.

[9] M. Popa, Multilinear function series in conditionally free probability with amalgamation, Commun. Stoch. Anal. 2 (2008), no. 2, 307-322.

[10] P. Skoufranis, Independences and Partial R-Transforms in Bi-Free Probability, Ann. Inst. Henri Poincaré Probab. Stat. 52 (2016), no. 3, 1437-1473.

[11] P. Skoufranis, On Operator-Valued Bi-Free Distributions, Adv. Math. 303 (2016), 638-715.

[12] R. Speicher, Combinatorial theory of the free product with amalgamation and operator-valued free probability theory, Memoirs of the American Mathematical Society, vol. 627, American Mathematical Society, 1998.

[13] D. Voiculescu, Operations on certain non-commutative operator-valued random variables, Astérisque 232 (1995), $243-275$. 
[14] D. Voiculescu, Free probability for pairs of faces I, Comm. Math. Phys. 332 (2014), no. 3, 955-980.

Department of Mathematics and Statistics, Queen's University, Jeffrey Hall, Kingston, Ontario, K7L 3N6, Canada

E-mail address: gu.y@queensu.ca

Department of Mathematics and Statistics, York University, 4700 Keele Street, Toronto, Ontario, M3J 1P3, Canada

E-mail address: pskoufra@yorku.ca 